\title{
Hadamard regularization
}

Luc Blanchet ${ }^{\mathrm{a}}$ )

Département d'Astrophysique Relativiste et de Cosmologie, Centre National de la Recherche Scientifique (UMR 8629), Observatoire de Paris, 92195 Meudon Cedex, France and Theoretical Astrophysics, California Institute of Technology, Pasadena, California 91125

Guillaume Faye

Département d'Astrophysique Relativiste et de Cosmologie, Centre National de la Recherche Scientifique (UMR 8629), Observatoire de Paris, 92195 Meudon Cedex, France

(Received 10 April 2000; accepted for publication 7 July 2000)

Motivated by the problem of the dynamics of point-particles in high postNewtonian (e.g., 3PN) approximations of general relativity, we consider a certain class of functions which are smooth except at some isolated points around which they admit a power-like singular expansion. We review the concepts of (i) Hadamard "partie finie" of such functions at the location of singular points, (ii) the partie finie of their divergent integral. We present and investigate different expressions, useful in applications, for the latter partie finie. To each singular function, we associate a partie-finie (Pf) pseudo-function. The multiplication of pseudofunctions is defined by the ordinary (pointwise) product. We construct a deltapseudo-function on the class of singular functions, which reduces to the usual notion of Dirac distribution when applied on smooth functions with compact support. We introduce and analyze a new derivative operator acting on pseudofunctions, and generalizing, in this context, the Schwartz distributional derivative. This operator is uniquely defined up to an arbitrary numerical constant. Time derivatives and partial derivatives with respect to the singular points are also investigated. In the course of the paper, all the formulas needed in the application to the physical problem are derived. (C) 2000 American Institute of Physics. [S0022-2488(00)03710-5]

\section{INTRODUCTION}

The Hadamard regularization, ${ }^{1,2}$ based on the concept of finite part ("partie finie") of a singular function or a divergent integral, plays an important role in several branches of Mathematical Physics (see Refs. 3-6 for reviews). Typically one deals with functions admitting some nonintegrable singularities on a discrete set of isolated points located at finite distances from the origin. The regularization consists of assigning by definition a value for the function at the location of one of the singular points, and for the (generally divergent) integral of that function. The definition may not be fully deterministic, as the Hadamard partie finie depends in general on some arbitrary constants. The Hadamard regularization is one among several other possible regularizations. ${ }^{4}$

A motivation for investigating the properties of a regularization comes from the physical problem of the gravitational interaction of compact bodies in general relativity. As it is hopeless to find a sufficiently general exact solution of this problem, we resort to successive post-Newtonian approximations (limit $c \rightarrow+\infty$ ). Within the post-Newtonian framework, it makes sense to model compact objects like black holes by point-like particles. This is possible at the price of introducing a regularization, in order to cure the divergencies due to the infinite self-field of the point-masses. However, general relativity is a nonlinear theory and, if we want to go to high post-Newtonian

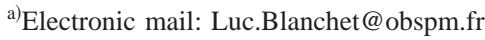


approximations, involving high nonlinear terms, the process of regularization must be carefully defined. In particular, it turns out that, from the third-post-Newtonian approximation (3PN or $\left.1 / c^{6}\right)$, the problem becomes complicated enough that a rather sophisticated version of the Hadamard regularization, including a theory of generalized functions, is required. By contrast, a cruder form of the Hadamard regularization, using merely the concept of partie finie of singular functions ${ }^{7-13}$ is sufficient to treat the problem up to the $2 \mathrm{PN}$ order. Furthermore, we know that the answer provided by the Hadamard regularization up to the 2PN order is correct, in the sense that the field of the two bodies matches the inner field generated by two black holes, ${ }^{14}$ and the result for the equations of motion can be recovered without the need of any regularization from computations valid for extended nonsingular objects. ${ }^{15,16}$ Conforted by these observations we systematically investigate in this paper the Hadamard regularization as well as a theory of associated generalized functions, in a form which can be directly applied to the study of the dynamics of two point-like particles at the 3PN order. ${ }^{17}$ (We therefore restrict our attention to two singular points; however most of the results of the paper can be generalized to any number of points.) Notice that this problem enjoys a direct relevance to the future gravitational-wave experiments LIGO and VIRGO, which should be able to detect the radiation from black-hole and/or neutron-star binaries which a precision compatible with the 3PN approximation. ${ }^{18}$

Consider the class $\mathcal{F}$ of functions on $\mathbb{R}^{3}$ that are smooth except at two isolated singularities 1 and 2, around which they admit some power-like singular expansions. The Hadamard partie finie $(F)_{1}$ of $F \in \mathcal{F}$ at the location of singularity 1, as reviewed in Sec. II, is defined by the average over spatial directions of the finite-part coefficient in the expansion of $F$ around 1. On the other hand, the Hadamard partie finie $\operatorname{Pf} \int d^{3} \mathbf{x} F$ of the divergent integral of $F$, we will review in Sec. III, is obtained from the removal to the integral of the divergent part arising when two regularizing volumes surrounding the singularities shrink to zero. Both concepts of partie finie are closely related. Notably, the partie-finie integral of a gradient is equal to the sum of the parties finies (in the former sense) of the surface integrals surrounding the singularities, in the limit of vanishing areas. In Sec. IV we investivage several alternative expressions of the Hadamard partie finie of integrals, some of them based on a finite part defined by means of an analytic continuation process (see Ref. 2 for a relation between partie finie and analytic continuation). In our terminology, we adopt the name "partie finie" for the specific definitions due to Hadamard, and speak of a "finite part" when referring to other definitions, based for instance on analytic continuation. In Sec. V we focus to the case (important in applications) of the partie finie of a Poisson integral of $F \in \mathcal{F}$.

To any $F \in \mathcal{F}$, we associate in Sec. VI a generalized function, or partie-finie "pseudofunction" $\operatorname{Pf} F$, which is a linear form on $\mathcal{F}$ defined for any $G \in \mathcal{F}$ by the duality bracket $\langle\operatorname{Pf} F, G\rangle=\operatorname{Pf} \int d^{3} \mathbf{x} F G$. When restricted to the set $\mathcal{D}$ of smooth functions with compact support the pseudo-function $\operatorname{Pf} F$ is a distribution in the sense of Schwartz ${ }^{2}$ (see also Refs. 19-21 for more details about generalized functions and distributions), i.e., a linear form which is continuous with respect to the Schwartz topology. (However, we do not attempt here to introduce a topology on $\mathcal{F}$; we simply define the set of algebraic and differential rules, needed in applications, that are satisfied by the pseudo-functions on $\mathcal{F}$.) The product of pseudo-functions coincides with the ordinary ("pointwise") product used in physics, namely $\operatorname{Pf} F \cdot \operatorname{Pf} G=\operatorname{Pf}(F G)$. An important particular case is the pseudo-function $\operatorname{Pf} \delta_{1}$ obtained (in Sec. VI) from the pseudo-function associated with the Riesz delta-function, ${ }^{22}$ and that satisfies $\forall G \in \mathcal{F},\left\langle\operatorname{Pf} \delta_{1}, G\right\rangle=(G)_{1}$. The "Dirac pseudofunction" $\operatorname{Pf} \delta_{1}$ plays in the present context the same role as plays the Dirac measure in distribution theory. We introduce also more complicated objects such as $\operatorname{Pf}\left(F \delta_{1}\right)$. In Secs. VII and VIII we show how to construct a derivative operator on $\mathcal{F}$, generalizing for this class of function the standard distributional derivative operator on $\mathcal{D}$ and satisfying basically the so-called rule of integration by parts, namely $\forall F, G \in \mathcal{F},\left\langle\partial_{i}(\operatorname{Pf} F), G\right\rangle=-\left\langle\partial_{i}(\operatorname{Pf} G), F\right\rangle$. In addition we require that the derivative reduces to the "ordinary" derivative for functions that are bounded in a neighborhood of the singular points, and that the rule of commutation of derivatives holds. We find that this derivative operator is uniquely defined modulo a dependence on an arbitrary numerical constant (see Theorem 4 in Sec. VIII). It represents a natural notion of derivative within the context of Hadamard regularization of the functions in $\mathcal{F}$. However, it does not satisfy in general the Leibniz 
rule for the derivative of a product (in agreement with a theorem of Schwartz ${ }^{23}$ ). See Colombeau ${ }^{24}$ for a multiplication of distributions and associated distributional derivative satisfying the Leibniz rule. Further, we obtain the rules obeyed by the new derivative operator when acting on pseudofunctions such as $\operatorname{Pf}\left(F \delta_{1}\right)$ in Sec. VII, and we investigate the associated Laplacian operator in Sec. VIII. Finally, in Sec. IX, we consider the case of partial derivatives with respect to the singular points 1 and 2, as well as the time derivative when both singular points depend on time (i.e., represent the trajectories of real particules). Within this approach, the latter distributional derivative constitutes an important tool when studying the problem of the gravitational dynamics of point-particles at the $3 \mathrm{PN}$ order. ${ }^{17}$

Notation: $\mathbb{N}, \mathbb{Z}, \mathbb{R}$ and $\mathrm{C}$ are the usual sets of non-negative integers, integers, real numbers and complex numbers; $\mathbb{R}^{+*}$ is the set of strictly positive real numbers $s>0 ; \mathbb{R}^{3}$ is the usual threedimensional space endowed with the Euclidean norm $|\mathbf{x}|=\left(x_{1}^{2}+x_{2}^{2}+x_{3}^{2}\right)^{1 / 2} ; C^{p}(\Omega)$ is the set of $p$-times continuously differentiable functions on the open set $\Omega(p \leqslant+\infty) ; L_{\mathrm{loc}}^{1}(\Omega)$ is the set of locally integrable functions on $\Omega$; the $o$ and $O$ symbols for remainders have their standard meaning; distances between the field point $\mathbf{x}$ and the source points $\mathbf{y}_{1}$ and $\mathbf{y}_{2}$ are denoted by $r_{1}=\mid \mathbf{x}$ $-\mathbf{y}_{1} \mid$ and $r_{2}=\left|\mathbf{x}-\mathbf{y}_{2}\right| ;$ unit directions are $\mathbf{n}_{1}=\left(\mathbf{x}-\mathbf{y}_{1}\right) / r_{1}$ and $\mathbf{n}_{2}=\left(\mathbf{x}-\mathbf{y}_{2}\right) / r_{2} ; d \Omega_{1}$ and $d \Omega_{2}$ are the solid angle elements associated with $\mathbf{n}_{1}$ and $\mathbf{n}_{2} ; r_{12}=\left|\mathbf{y}_{1}-\mathbf{y}_{2}\right| ; \mathcal{B}_{1}(s)$ and $\mathcal{B}_{2}(s)$ denote the closed spherical balls of radius $s$ centered on $\mathbf{y}_{1}$ and $\mathbf{y}_{2} ; \partial_{i}=\partial / \partial x^{i},{ }_{1} \partial_{i}=\partial / \partial y_{1}^{i},{ }_{2} \partial_{i}=\partial / \partial y_{2}^{i} ; L$ $=i_{1} i_{2} \cdots i_{l}$ is a multi-index with length $l ; n_{1}^{L}=n_{1}^{i_{1}} \cdots n_{1}^{i_{l}}$ and $\partial_{L}=\partial_{i_{1}} \cdots \partial_{i_{l}}$; the symmetric-trace-free (STF) projection is denoted by $\hat{n}_{1}^{L}=\operatorname{STF}\left(n_{1}^{L}\right) ;(i j)=(i j+j i) / 2$ and $[i j]=(i j-j i) / 2 ; 1 \leftrightarrow 2$ means the same expression but corresponding to the point 2 ; for clearer reading, we use left-side labels 1 and 2 when the quantity appears within the text, like for the partial derivatives ${ }_{1} \partial_{i}$ and ${ }_{2} \partial_{i}$ or the coefficients ${ }_{1} f_{a}$ and ${ }_{2} f_{b}$, and labels placed underneath the quantity when it appears in an equation; iff means if and only if.

\section{HADAMARD PARTIE FINIE}

\section{A. A class of singular functions}

All over this paper we consider the class of functions of a "field" point $\mathbf{x} \in \mathbb{R}^{3}$ that are singular at the location of two "source" points $\mathbf{y}_{1}$ and $\mathbf{y}_{2}$ around which they admit some singular expansions.

Definition 1: A real function $F(\mathbf{x})$ on $\mathbb{R}^{3}$ is said to belong to the class of functions $\mathcal{F}$ iff:

(i) $\quad F$ is smooth on $\mathbb{R}^{3}$ deprived from $\mathbf{y}_{1}$ and $\mathbf{y}_{2}$, i.e., $F \in C^{\infty}\left(\mathbb{R}^{3}-\left\{\mathbf{y}_{1}, \mathbf{y}_{2}\right\}\right)$.

(ii) There exists an ordered family of indices $\left(a_{i}\right)_{i \in \mathbb{N}}$ with $a_{i} \in \mathbb{R}$, and a family of coefficients ${ }_{1} f_{a_{i}}$, such that

$$
\forall N \in \mathbb{N}, \quad F(\mathbf{x})=\sum_{i=0}^{i_{N}} r_{1}^{a_{i}} f_{a_{i}}\left(\mathbf{n}_{1}\right)+R_{N}(\mathbf{x}) .
$$

Here $r_{1}=\left|\mathbf{x}-\mathbf{y}_{1}\right|$ and $\mathbf{n}_{1}=\left(\mathbf{x}-\mathbf{y}_{1}\right) / r_{1} ; i_{N}$ satisfies $a_{0}<a_{1}<\cdots<a_{i_{N}} \leqslant N<a_{i_{N}+1}$; and the "remainder" is

$$
R_{N}(\mathbf{x})=o\left(r_{1}^{N}\right), \quad \text { when } r_{1} \rightarrow 0 .
$$

(iii) Idem with indices $\left(b_{i}\right)_{i \in \mathbb{N}}$, coefficients ${ }_{2} f_{b_{i}}$, remainder ${ }_{2} R_{N}, r_{1} \leftrightarrow r_{2}$ and $\mathbf{n}_{1} \leftrightarrow \mathbf{n}_{2}$.

In addition to Definition 1, we always assume that the functions $F \in \mathcal{F}$ decrease sufficiently fast at infinity (when $|\mathbf{x}| \rightarrow \infty$ ) so that all integrals we meet are convergent at infinity. Thus, when discussing the integral $\int d^{3} \mathbf{x} F$, we suppose implicitly that $F=o\left(|\mathbf{x}|^{-3}\right)$ at infinity [or sometimes $F=O\left(|\mathbf{x}|^{-3-\epsilon}\right)$ where $\left.\epsilon>0\right]$, so that the possible divergencies come only from the bounds at the singular points $\mathbf{y}_{1,2}$. Similarly, when considering the integral $\int d^{3} \mathbf{x} F G$, we suppose $F G$ $=o\left(|\mathbf{x}|^{-3}\right)$, but for instance we allow $F$ to blow up at infinity, say $F=O(|\mathbf{x}|)$, if we know that $G$ decreases rapidly, e.g., $G=o\left(|\mathbf{x}|^{-4}\right)$; in the case of $\int d^{3} \mathbf{x} \partial_{i} F$, we generally assume $F$ $=o\left(|\mathbf{x}|^{-2}\right)$. [Clearly, from Definition 1 the ordinary product $F G$ of two functions of $\mathcal{F}$ is again a function of $\mathcal{F}$; and similarly the ordinary gradient $\partial_{i} F \in \mathcal{F}$.] 
An important assumption in Definition 1 is that the powers of $r_{1}$ in the expansion of $F$ when $r_{1} \rightarrow 0$ (and similarly when $r_{2} \rightarrow 0$ ) are bounded from below, i.e., $a_{0} \leqslant a_{i}$ where the most "divergent" power of $r_{1}$, which clearly depends on $F$, is $a_{0}=a_{0}(F)$. Thus the part of the expansion which diverges when $r_{1} \rightarrow 0$ is composed of a finite number of terms. Notice also that we have excluded in Definition 1 the possible appearance of logarithms of $r_{1}$ (or $r_{2}$ ) in the expansion of $F$. See Sellier ${ }^{5}$ for a more general study in the case where some arbitrary powers of logarithms are present. We will discuss the occurrence of logarithms in Sec. V, when dealing with the Poisson integral of $F$. At last, we point out that the coefficients ${ }_{1} f_{a}$ (and similarly ${ }_{2} f_{b}$ ) do not depend only on $\mathbf{n}_{1}$, but also they do on the source points $\mathbf{y}_{1}$ and $\mathbf{y}_{2}$, so that in principle we should write ${ }_{1} f_{a}\left(\mathbf{n}_{1} ; \mathbf{y}_{1}, \mathbf{y}_{2}\right)$; however, for simplicity's sake we omit writing the dependence on the source points. The coefficients could also depend on other variables such as the velocities $\mathbf{v}_{1}$ and $\mathbf{v}_{2}$ of the source points, but the velocities do not participate in the process of regularization and can be ignored for the moment (we will return to this question in Section IX when considering the time dependence of $F$ ).

Once the class $\mathcal{F}$ has been defined, we shall often write in this paper the expansions of $F$ when $r_{1,2} \rightarrow 0$ in the simplified forms

$$
\begin{aligned}
& F(\mathbf{x})=\sum_{a_{0} \leqslant a \leqslant N} r_{1}^{a} f_{a}\left(\mathbf{n}_{1}\right)+o\left(r_{1}^{N}\right) \quad \text { when } r_{1} \rightarrow 0, \\
& F(\mathbf{x})=\sum_{b_{0} \leqslant b \leqslant N} r_{2}^{b} f_{b}\left(\mathbf{n}_{2}\right)+o\left(r_{2}^{N}\right) \quad \text { when } r_{2} \rightarrow 0,
\end{aligned}
$$

by which we really mean the expansions in Definition 1, i.e., in particular where the indices $a$ $\in\left(a_{i}\right)_{i \in \mathbb{N}}$ and $b \in\left(b_{i}\right)_{i \in \mathbb{N}}$, and are a priori real. However, most of the time (in applications), it is sufficient to assume that the powers of $r_{1,2}$ are relative integers $a, b \in \mathbb{Z}$. We can then write the expansion $r_{1} \rightarrow 0$ in the form

$$
F=\sum_{k=0}^{k_{0}} 1 / r_{1}^{(1+k)} f_{-1-k}+\sum_{k=0}^{N} r_{1}^{k} f_{k}+o\left(r_{1}^{N}\right)
$$

where $k_{0}=-1-a_{0}$. In the following we shall sometimes derive the results in the simpler case where the powers $\in \mathbb{Z}$, being always undertood that the generalization to the case of real powers is straightforward. Finally, it is worth noting that the assumption (i) in Definition 1 , that $F$ is $C^{\infty}$ outside $\left\{\mathbf{y}_{1}, \mathbf{y}_{2}\right\}$, can often be relaxed to allow some functions to have integrable singularities. An example is the function $\mathbf{x} \rightarrow 1 /\left|\mathbf{x}-\mathbf{x}^{\prime}\right|$ encountered in Sec. V, depending on a fixed "spectator", point $\mathbf{x}^{\prime}$ distinct from $\mathbf{y}_{1}$ and $\mathbf{y}_{2}$. To treat such objects, we introduce a larger class of functions, $\mathcal{F}_{\text {loc }}$.

Definition 2: $F(\mathbf{x})$ is said to belong to the class of functions $\mathcal{F}_{\text {loc }}$ iff:

$\left(i^{\prime}\right) F$ is locally integrable on $\mathbb{R}^{3}$ deprived from $\mathbf{y}_{1}$ and $\mathbf{y}_{2}$, i.e., $F \in L_{\text {loc }}^{1}\left(\mathbb{R}^{3}-\left\{\mathbf{y}_{1}, \mathbf{y}_{2}\right\}\right)$.

(ii)-(iii) in Definition 1 hold.

For simplicity, in the following, we shall derive most of the results for functions belonging to the class $\mathcal{F}$ (even if the generalization to $\mathcal{F}_{\text {loc }}$ is trivial); $\mathcal{F}_{\text {loc }}$ will be employed only occasionally.

\section{B. Partie finie of a singular function}

The first notion of Hadamard partie finie is that of a singular function at the very location of one of its singular points.

Definition 3: Given $F \in \mathcal{F}$ we define the Hadamard partie finie of $F$ at the point $\mathbf{y}_{1}$ to be

$$
(F)_{1}=\int \frac{d \Omega_{1}}{4 \pi} f_{0}\left(\mathbf{n}_{1}\right),
$$


where $d \Omega_{1}=d \Omega\left(\mathbf{n}_{1}\right)$ denotes the solid angle element of origin $\mathbf{y}_{1}$ and direction $\mathbf{n}_{1}$.

In words, the partie finie of $F$ at point 1 is defined by the angular average, with respect to the unit direction $\mathbf{n}_{1}$, of the coefficient of the zeroth power of $r_{1}$ in the expansion of $F$ near 1 (and similarly for the point 2). There is a nonzero partie finie only if the family of indices $\left(a_{i}\right)_{i \in \mathbb{N}}$ in Definition 1 contains the value 0, i.e., $\exists i_{0}$ such that $a_{i_{0}}=0$. The latter definition applied to the product $F G$ of two functions in $\mathcal{F}$ yields

$$
(F G)_{1}=\sum_{a_{0}(F) \leqslant a \leqslant-a_{0}(G)} \int \frac{d \Omega_{1}}{4 \pi f_{a} g_{-a},}
$$

where ${ }_{1} f_{a}$ and ${ }_{1} g_{a}$ are the coefficients in the expansions of $F$ and $G$ when $r_{1} \rightarrow 0$ (the summation over $a$ is always finite). From (2.6) it is clear that the Hadamard partie finie is not "distributive" with respect to the multiplication, in the sense that

$$
(F G)_{1} \neq(F)_{1}(G)_{1} \quad \text { in general. }
$$

The partie finie picks up the angular average of $f_{1}\left(\mathbf{n}_{1}\right)$, namely the scalar or $l=0$ piece in the spherical-harmonics expansion $\left(Y_{l m}\right)$, or, equivalently, in the expansion on the basis of symmetric and trace-free $(\mathrm{STF})$ products of unit vectors $\mathbf{n}_{1}=\left(n_{1}^{i}\right)$. For any $l \in \mathbb{N}$, we denote by $L$ $=i_{1} i_{2} \cdots i_{l}$ a multi-index composed of $l$ indices, and similarly $L-1=i_{1} i_{2} \cdots i_{l-1}, P=j_{1} j_{2} \cdots j_{p}$. In general we do not need to specify the carrier index $i$ or $j$, so a tensor with $l$ upper indices is denoted $T^{L}$, and for instance the scalar formed by contraction with another tensor $U^{L}$ of the same type is written as $S=T^{L} U^{L}=T^{i_{1} \cdots i_{l}} U^{i_{1} \cdots i_{l}}$, where we omit writing the $l$ summations over the $l$ indices $i_{k}=1,2,3$. We denote a product of $l$ components of the unit vector $n_{1}^{i}$ by $n_{1}^{L}=n_{1}^{i_{1}} \cdots n_{1}^{i_{l}}$, and the STF projection of that product by $\hat{n}_{1}^{L} \equiv \operatorname{STF}\left(n_{1}^{L}\right)$ : e.g., $\hat{n}_{1}^{i j}=n_{1}^{i} n_{1}^{j}-\frac{1}{3} \delta^{i j}, \quad \hat{n}_{1}^{i j k}=n_{1}^{i} n_{1}^{j} n_{1}^{k}$ $-\frac{1}{5}\left(n_{1}^{i} \delta^{j k}+n_{1}^{j} \delta^{k i}+n_{1}^{k} \delta^{i j}\right)$. More generally, we denote by $\hat{T}^{L}$ the STF projection of $T^{L}$; that is, $\hat{T}^{L}$ is symmetric, and satisfies $\delta_{i_{l-1}{ }^{i} l} \hat{T}^{i_{l-1}{ }^{i} l}{ }^{L-2}=0$ (see Ref. 25 and Appendix A of Ref. 26 for a compendium of formulas using the STF formalism). The coefficients ${ }_{1} f_{a}$ of the expansion of $F$ admit the STF decomposition

$$
f_{a}\left(\mathbf{n}_{1}\right)=\sum_{l=0}^{+\infty} n_{1}^{L} \hat{f}_{a}^{L}
$$

where the ${ }_{1} \hat{f}_{a}^{L}$, s are constant STF tensors, given by the inverse formula:

$$
\hat{f}_{a}^{L}=\frac{(2 l+1) ! !}{l !} \int \frac{d \Omega_{1}}{4 \pi} \hat{n}_{1}^{L} f_{a}\left(\mathbf{n}_{1}\right) .
$$

In STF notation, the Hadamard partie finie of $F$ at 1 reads simply as

$$
(F)_{1}=\hat{f}_{0},
$$

where ${ }_{1} \hat{f}_{a}$ denotes the first term in the expansion (2.8).

Lemma 1: The partie finie at 1 of the gradient $\partial_{i} F$ (as defined outside the singularities) of any function $F \in \mathcal{F}$ satisfies

$$
\left(\partial_{i} F\right)_{1}=3\left(\frac{n_{1}^{i}}{r_{1}} F\right)_{1} .
$$

This Lemma is particularly useful as it permits replacing systematically the differential operator $\partial_{i}$ by the algebraic one $3\left(n_{1}^{i} / r_{1}\right)$ when working under the partie-finie sign $(\cdots)_{1}$. 
Proof: The expansion when $r_{1} \rightarrow 0$ of the gradient is readily obtained from the expansion of $F$ itself as

$$
\partial_{i} F=\sum_{a} r_{1}^{a-1}\left[\begin{array}{cc}
a & n_{1}^{i} f_{a}+d_{1}{ }^{i} f_{a}
\end{array}\right]
$$

(with over-simplified notation for the sum), where the operator $d_{1}^{i}$ is defined as $r_{1} \partial_{i}$ when applied on a function of the sole unit vector $\mathbf{n}_{1}$. Hence, explicitly, $d_{1}^{i}=\left(\delta^{i j}-n_{1}^{i j}\right)\left(\partial / \partial n_{1}^{j}\right)$. This operator is evidently transverse to $\mathbf{n}_{1}: n_{1}^{i} d_{1}^{i}=0$, and we get, from the decomposition (2.8),

$$
d_{1}^{i} f_{a}=\sum_{l=0}^{+\infty} l\left(n_{1}^{L-1} \hat{f}_{1}^{i L-1}-n_{1}^{i L} \hat{f}_{a}^{L}\right) .
$$

Thus, by averaging over angles,

$$
\int \frac{d \Omega_{1}}{4 \pi} d_{1}^{i} f_{a}=\frac{2}{3} \hat{f}_{a}^{i}=2 \int \frac{d \Omega_{1}}{4 \pi} n_{1}^{i} f_{a} .
$$

We readily deduce that the partie finie of the gradient (2.12) is given by

$$
\left(\partial_{i} F\right)_{1}=3 \int \frac{d \Omega_{1}}{4 \pi} n_{1}^{i} f_{1}=\hat{f}_{1}^{i} \quad(\mathrm{QED}) .
$$

As an example of the application of Lemma 1, we can write, using an operation by parts, $\left(r_{1}^{3} \partial_{i} F\right)_{1}=\left[\partial_{i}\left(r_{1}^{3} F\right)-\partial_{i}\left(r_{1}^{3}\right) F\right]_{1}=\left[3 n_{1}^{i} r_{1}^{2} F-\partial_{i}\left(r_{1}^{3}\right) F\right]_{1}$, from which it follows that

$$
\left(r_{1}^{3} \partial_{i} F\right)_{1}=0 .
$$

Another consequence of Lemma 1, resulting from two operations by parts, is $\left(r_{1}^{2} \Delta F\right)_{1}$ $=\left[3 n_{1}^{i} r_{1} \partial_{i} F-\partial_{i}\left(r_{1}^{2}\right) \partial_{i} F\right]_{1}=\left(n_{1}^{i} r_{1} \partial_{i} F\right)_{1}=\left[3 F-\partial_{i}\left(n_{1}^{i} r_{1}\right) F\right]_{1}$ (where the Laplacian $\left.\Delta=\partial_{i} \partial_{i}\right)$, hence the identity

$$
\left(r_{1}^{2} \Delta F\right)_{1}=0 .
$$

By the same method we obtain also

$$
\left(\partial_{i j} F\right)_{1}=\left(\frac{15 n_{1}^{i j}-3 \delta^{i j}}{r_{1}^{2}} F\right)_{1}=\underset{1}{2\left(\hat{f}_{2}^{i j}+\delta_{1}^{i j} \hat{f}_{2}\right),}
$$

the right-hand side of the last equality being expressed in terms of the STF tensors parametrizing (2.8). Tracing out the previous formula, we find

$$
(\Delta F)_{1}=\left(\frac{6}{r_{1}^{2}} F\right)_{1}=\underset{1}{6} \hat{f}_{2} .
$$

Finally, let us quote the general formula for the partie finie of the $l$ th derivative $\partial_{L} F$ $=\partial_{i_{1}} \cdots \partial_{i_{l}} F$ :

$$
\left(\partial_{L} F\right)_{1}=l ! \sum_{k=0}^{[l / 2]} \delta_{1}^{(2 K} \hat{f}_{l}^{L-2 K)}
$$


Here, [l/2] denotes the integer part of $l / 2, \delta^{2 K}$ is the product of Kronecker symbols $\delta^{i_{1} i_{2}} \delta^{i_{3} i_{4}} \ldots \delta^{i_{2 k-1} i_{2 k}}$, and ${ }_{1} \hat{f}_{l}^{L-2 K}={ }_{1} \hat{f}_{l}^{i_{2 k+1} \cdots i_{l}}$; the parentheses around the indices denote the symmetrization. One may define the "regular" part of the function $F$ near the singularity 1 as the formal Taylor expansion when $r_{1} \rightarrow 0$ obtained using (2.20). Thus,

$$
F_{1}^{\mathrm{reg}} \equiv \sum_{l=0}^{+\infty} \frac{1}{l !} r_{1}^{l} n_{1}^{L}\left(\partial_{L} F\right)_{1}=\sum_{l=0}^{+\infty} r_{1}^{l} \sum_{k=0}^{[l / 2]} n_{1}^{L-2 K} \hat{f}_{l}^{L-2 K}
$$

\section{PARTIE-FINIE INTEGRALS}

\section{A. The partie finie of a divergent integral}

The second notion of Hadamard partie finie is that of the integral $\int d^{3} \mathbf{x} F(\mathbf{x})$, where $F \in \mathcal{F}$. This integral is generally divergent because of the presence of the singular points $\mathbf{y}_{1}$ and $\mathbf{y}_{2}$ (recall that we always assume that the function decreases sufficiently rapidly at infinity so that we never have any divergency coming from the integration bound $|\mathbf{x}| \rightarrow+\infty)$. Consider first the domain $\mathbb{R}^{3}$ deprived from two spherical balls $\mathcal{B}_{1}(s)$ and $\mathcal{B}_{2}(s)$ of radius $s$, centered on the two singularities $\mathbf{y}_{1}, \mathbf{y}_{2}: \mathcal{B}_{1}(s)=\left\{\mathbf{x} ; r_{1} \leqslant s\right\}$ and $\mathcal{B}_{2}(s)=\left\{\mathbf{x} ; r_{2} \leqslant s\right\}$. We assume that $s$ is small enough, i.e., $s$ $<r_{12} / 2$ where $r_{12}=\left|\mathbf{y}_{1}-\mathbf{y}_{2}\right|$, so that the two balls do not intersect. For $s>0$ the integral over this domain, say $I(s)=\int_{\mathrm{R}^{3} \backslash \mathcal{B}_{1}(s) \cup \mathcal{B}_{2}(s)} d^{3} \mathbf{x} F$, is well-defined and generally tends to infinity when $s$ $\rightarrow 0$. Thanks to the expansions (assumed in Definition 1) of $F$ near the singularities, we easily compute the part of $I(s)$ that blows up when $s \rightarrow 0$; we find that this divergent part is given, near each singularity, by a finite sum of strictly negative powers of $s$ (a polynomial of $1 / s$ in general) plus a term involving the logarithm of $s$. By subtracting from $I(s)$ the corresponding divergent part, we get a term that possesses a finite limit when $s \rightarrow 0$; the Hadamard partie finie ${ }^{1}$ is defined as this limit. Associated with the logarithm of $s$, there arises an ambiguity which can be viewed as the freedom in the re-definition of the unit system we employ to measure the length $s$. In fact it is convenient to introduce two constant length scales $s_{1}$ and $s_{2}$, one per singularity, in order to a-dimensionalize the logarithms as $\ln \left(s / s_{1}\right)$ and $\ln \left(s / s_{2}\right)$.

Definition 4: For any $F \in \mathcal{F}$ integrable in a neighborhood of $|\mathbf{x}|=+\infty$, we define the Hadamard partie finie of the divergent integral $\int d^{3} \mathbf{x} F$ as

$$
\mathrm{Pf}_{s_{1}, s_{2}} \int d^{3} \mathbf{x} F=\lim _{s \rightarrow 0}\left\{\int_{\mathbb{R}^{3} \backslash \mathcal{B}_{1}(s) \cup \mathcal{B}_{2}(s)} d^{3} \mathbf{x} F+\sum_{a+3<0} \frac{s^{a+3}}{a+3} \int d \Omega_{1} f_{a}+\ln \left(\frac{s}{s_{1}}\right) \int d \Omega_{1} f_{-3}+1 \leftrightarrow 2\right\},
$$

where $1 \leftrightarrow 2$ means the same previous two terms but concerning the singularity 2 .

This notion of partie finie can be extended to functions which are locally integrable outside the singularities, i.e., $F \in \mathcal{F}_{\text {loc }}$ (see Definition 2). In (3.1) the divergent terms are composed of a sum over $a$ such that $a+3<0$ as well as a logarithmic term, by which we really mean, using the more detailed notation of Definition 1 ,

$$
\sum_{i=0}^{i_{l}-1} \frac{s^{a_{i}+3}}{a_{i}+3} \int d \Omega_{1} f_{a_{i}}+\delta_{-3, a_{i_{l}}} \ln \left(\frac{s}{s_{1}}\right) \int d \Omega_{1} f_{a_{i_{l}}}+1 \leftrightarrow 2,
$$

where $i_{l}$ is such that $a_{0}<a_{1}<\cdots<a_{i_{l}-1}<-3 \leqslant a_{i_{l}}$ (the sum is always finite); we have introduced a Kronecker symbol $\delta_{-3, a_{i_{l}}}$ to recall that the logarithm is present only if the family of indices $\left(a_{i}\right)_{i \in \mathbb{N}}$ contains the integer -3 (i.e., $a_{i_{l}}=-3$ ). The divergent terms in (3.1) can also be expressed by means of the partie finie defined by (2.5). Indeed, they read as

$$
4 \pi\left[\sum_{a+3<0} \frac{s^{a+3}}{a+3}\left(\frac{F}{r_{1}^{a}}\right)_{1}+\ln \left(\frac{s}{s_{1}}\right)\left(r_{1}^{3} F\right)_{1}\right]+1 \leftrightarrow 2
$$


[coming back to the less detailed notation of (3.1)].

The partie-finie integral (3.1) depends intrinsically on the two arbitrary constants $s_{1}$ and $s_{2}$ introduced above. There is another way to interpret these constants besides the necessity to take into account the dimension of $s$, which is discussed by Sellier in Ref. 5. With this point of view we initially define the partie finie using two arbitrarily shaped volumes $\mathcal{V}_{1}$ and $\mathcal{V}_{2}$ instead of the two spherical balls $\mathcal{B}_{1}$ and $\mathcal{B}_{2}$. Consider for instance the two volumes $\mathcal{V}_{1}=\left\{\mathbf{x} ; r_{1} \leqslant s \rho_{1}\left(\mathbf{n}_{1}\right)\right\}$ and $\mathcal{V}_{2}=\left\{\mathbf{x} ; r_{2} \leqslant s \rho_{2}\left(\mathbf{n}_{2}\right)\right\}$, where $s \in \mathbb{R}^{+*}$ measures the size of the volumes and the two functions $\rho_{1}$ and $\rho_{2}$ describe their shape (the balls $\mathcal{B}_{1}$ and $\mathcal{B}_{2}$ corresponding simply to $\rho_{1}$ and $\rho_{2} \equiv 1$ ). Here, we assume for simplicity that the volumes remain isometric to themselves when $s$ varies. Then, the partie finie is defined as the limit of the integral over $\mathbb{R}^{3} \backslash \mathcal{V}_{1} \cup \mathcal{V}_{2}$ to which we subtract the corresponding divergent terms when $s \rightarrow 0$, without adding any normalizing constant to the logarithms. In this way, we find that the alternative definition is equivalent to our definition (3.1) provided that $s_{1}$ and $s_{2}$ are related to the shapes of the regularizing volumes $\mathcal{V}_{1}$ and $\mathcal{V}_{2}$ through the formula

$$
\ln s_{1} \int d \Omega_{1} f_{-3}=\int d \Omega_{1} f_{-3} \ln \rho_{1}
$$

(and similarly for $s_{2}$ ). The arbitrariness on the two original regularizing volumes is therefore encoded into the two (and only two) constants $s_{1}$ and $s_{2}$. A closely related way to interpret them is linked to the necessity to allow the change of the integration variable $\mathbf{x}$ in the integral $\int d^{3} \mathbf{x} F$. Such an operation modifies the size and shape of the regularizing volumes, thus the balls $\mathcal{B}_{1}$ and $\mathcal{B}_{2}$ are in general transformed into some new volumes $\mathcal{V}_{1}$ and $\mathcal{V}_{2}$; so, according to the previous argument, the freedom of choosing the integration variable reflects out in the freedom of choosing two arbitrary constants $s_{1}$ and $s_{2}$. (In this paper we shall assume that $s_{1}$ and $s_{2}$ are fixed once and for all.)

An alternative expression of the Hadamard partie finie is often useful because it does not involve the limit $s \rightarrow 0$, but is written with the help of a finite parameter $s^{\prime} \in \mathbb{R}^{+*}$. Consider some $s^{\prime}$ such that $0<s<s^{\prime}$, and next, split the integral over $\mathbb{R}^{3} \backslash \mathcal{B}_{1}(s) \cup \mathcal{B}_{2}(s)$ into the sum of the integral over $\mathbb{R}^{3} \backslash \mathcal{B}_{1}\left(s^{\prime}\right) \cup \mathcal{B}_{2}\left(s^{\prime}\right)$ and the two integrals over the ring-shaped domains $\mathcal{B}_{1}\left(s^{\prime}\right) \backslash \mathcal{B}_{1}(s)$ and $\mathcal{B}_{1}\left(s^{\prime}\right) \backslash \mathcal{B}_{1}(s)$. If $s<s^{\prime} \ll 1$ we can substitute, respectively, into the ring-shaped integrals the expansions of $F$ when $r_{1} \rightarrow 0$ and $r_{2} \rightarrow 0$ [see (2.3)]. The terms that are divergent in $s$ cancel out, so we can apply the limit $s \rightarrow 0$ (with fixed $s^{\prime}$ ). This yields the following expression for the partie finie: $\forall N \in \mathbb{N}$,

$$
\begin{aligned}
\mathrm{Pf}_{s_{1}, s_{2}} \int d^{3} \mathbf{x} F= & \int_{\mathbb{R}^{3} \backslash \mathcal{B}_{1}\left(s^{\prime}\right) \cup \mathcal{B}_{2}\left(s^{\prime}\right)} d^{3} \mathbf{x} F+\sum_{\substack{a+3 \leqslant N \\
a+3 \neq 0}} \frac{s^{\prime a+3}}{a+3} \int d \Omega_{1} f_{a}+\ln \left(\frac{s^{\prime}}{s_{1}}\right) \int d \Omega_{1} f_{-3} \\
& +1 \leftrightarrow 2+o\left(s^{\prime N+3}\right),
\end{aligned}
$$

which is valid for an arbitrary fixed $s^{\prime}$. Of course, up to any given finite order $N$ the second member of (3.3) depends on $s^{\prime}$, but in the formal limit $N \rightarrow+\infty$, this dependence disappears and, in fine, the partie finie is independent of $s^{\prime}$.

\section{B. Partie-finie integral of a gradient}

A fundamental feature of the Hadamard partie finie of a divergent integral is that the integral of a gradient $\partial_{i} F$ is a priori not zero, since the surface integrals surrounding the two singularities become infinite when the surface areas shrink to zero, and may possess a finite part.

Theorem 1: For any $F \in \mathcal{F}$ the partie finie of the gradient of $F$ is given by

$$
\operatorname{Pf} \int d^{3} \mathbf{x} \partial_{i} F=-4 \pi\left(n_{1}^{i} r_{1}^{2} F\right)_{1}+1 \leftrightarrow 2,
$$


where the singular value at point 1 is defined by (2.5).

In the case of a regular function, the result is always zero from the simple fact that the surface areas tend to zero-cf. the factor $r_{1}^{2}$ in the right side of (3.4). However, for $F \in \mathcal{F}$, the factor $r_{1}^{2}$ is in general compensated by a divergent term in the expansion of $F$, possibly producing a finite contribution.

Proof: We apply (3.1) to the case of the gradient $\partial_{i} F$, using the expansion of $\partial_{i} F$ when $r_{1}$ $\rightarrow 0$ as given by (2.12). The expression of the divergent terms is simplified with the help of the identity (2.14), which shows notably that the logarithms and associated constants $s_{1,2}$ disappear. This leads to

$$
\lim _{s \rightarrow 0}\left\{\int_{\mathbb{R}^{3} \backslash \mathcal{B}_{1}(s) \cup \mathcal{B}_{2}(s)} d^{3} \mathbf{x} \partial_{i} F+\sum_{a+2<0} s^{a+2} \int d \Omega_{1} n_{1}^{i} f_{a}+1 \leftrightarrow 2\right\} .
$$

Next, the first term inside the braces is transformed via the Gauss theorem into two surface integrals at $r_{1}=s$ and $r_{2}=s$, where we can replace $F$ by the corresponding expansions around $\mathbf{y}_{1}$ and $\mathbf{y}_{2}$, respectively. We get

$$
\lim _{s \rightarrow 0}\left\{-\sum_{a} s^{a+2} \int d \Omega_{1} n_{1}^{i} f_{a}+\sum_{a+2<0} s^{a+2} \int d \Omega_{1} n_{1}^{i} f_{a}\right\}=-\int d \Omega_{1} n_{1}^{i} f_{-2}
$$

(and similarly when $1 \leftrightarrow 2$ );

QED.

From Theorem 1 it results that the correct formula for "integrating by parts" under the sign Pf is

$$
\operatorname{Pf} \int d^{3} \mathbf{x} F \partial_{i} G=-\operatorname{Pf} \int d^{3} \mathbf{x} G \partial_{i} F-4 \pi\left(n_{1}^{i} r_{1}^{2} F G\right)_{1}-4 \pi\left(n_{2}^{i} r_{2}^{2} F G\right)_{2} .
$$

Note also that the partie-finie integrals of a double derivative as well as a Laplacian are given by

$$
\begin{gathered}
\operatorname{Pf} \int d^{3} \mathbf{x} \partial_{i j} F=4 \pi\left(r_{1}\left(\delta^{i j}-2 n_{1}^{i j}\right) F\right)_{1}+1 \leftrightarrow 2, \\
\operatorname{Pf} \int d^{3} \mathbf{x} \Delta F=4 \pi\left(r_{1} F\right)_{1}+1 \leftrightarrow 2 .
\end{gathered}
$$

\section{Parties finies and the Riesz delta-function}

The Riesz delta-function ${ }^{22}$ plays an important role in the context of Hadamard parties finies. It is defined for any $\varepsilon \in \mathbb{R}^{+*}$ by ${ }_{\varepsilon} \delta(\mathbf{x})=[\varepsilon(1-\varepsilon) / 4 \pi]|\mathbf{x}|^{\varepsilon^{-3}}$; when $\varepsilon \rightarrow 0$, it tends, in the usual sense of distribution theory, towards the Dirac measure in three dimensions-i.e., $\lim _{\varepsilon \rightarrow 0 \varepsilon} \delta=\delta$, as can be seen from the easily checked property that $\Delta\left(|\mathbf{x}|^{\varepsilon-1}\right)=-4 \pi_{\varepsilon} \delta(\mathbf{x})$. The point for our purpose is that when defined with respect to one of the singularities, the Riesz delta-function belongs to $\mathcal{F}$. Thus, let us set, $\forall \varepsilon \in \mathbb{R}^{+*}$,

$$
{ }_{\varepsilon} \delta_{1}(\mathbf{x}) \equiv{ }_{\varepsilon} \delta\left(\mathbf{x}-\mathbf{y}_{1}\right)=\frac{\varepsilon(1-\varepsilon)}{4 \pi} r_{1}^{\varepsilon-3} \in \mathcal{F}
$$

(and idem for 2). Now we can apply $\operatorname{to}_{\varepsilon} \delta_{1}(\mathbf{x})$ the previous definitions for parties finies. In particular, from Definition 3, we see that $\delta_{\varepsilon} \delta_{1}$ has no partie finie at 1 when $\varepsilon$ is small enough: $\left({ }_{\varepsilon} \delta_{1}\right)_{1}=0$. From Definition 4, we have the following.

Lemma 2: For any $F \in \mathcal{F}$, we have

$$
\lim _{\varepsilon \rightarrow 0} \operatorname{Pf} \int d^{3} \mathbf{x}_{\varepsilon} \delta_{1} F=(F)_{1}
$$


where the value of $F$ at point 1 is given by the prescription (2.5).

Proof: For $\varepsilon>0$ we evaluate the finite part of the integral for the product ${ }_{\varepsilon} \delta_{1} F \in \mathcal{F}$ using the specific form (3.3) of the partie finie defined in terms of a given finite $s^{\prime}$. The expansions of ${ }_{\varepsilon} \delta_{1} F$ when $r_{1,2}$ tend to zero are readily determined to be

$$
\begin{gathered}
{ }_{\varepsilon} \delta_{1} F=\frac{\varepsilon(1-\varepsilon)}{4 \pi} \sum_{a} r_{1}^{a+\varepsilon-3} f_{a}\left(\mathbf{n}_{1}\right) \quad \text { for } r_{1} \rightarrow 0, \\
\delta_{\varepsilon} F=\frac{\varepsilon(1-\varepsilon)}{4 \pi} \sum_{l \geqslant 0} \frac{(-)^{l}}{l !} \partial_{L} r_{12}^{\varepsilon-3} \sum_{b} r_{2}^{b+l} n_{2}^{L} f_{b}\left(\mathbf{n}_{2}\right), \quad \text { for } r_{2} \rightarrow 0 .
\end{gathered}
$$

In the second equation we used the Taylor expansion $r_{1}^{\varepsilon-3}=\Sigma_{l \geqslant 0}\left[(-)^{l} / l !\right] r_{2}^{l} n_{21}^{L} \partial_{L} r_{12}^{\varepsilon-3}$ when $r_{2} \rightarrow 0$, with the notation $n_{2}^{L}=n_{2}^{i_{1}} \cdots n_{2}^{i_{l}}$ and ${ }_{1} \partial_{L}={ }_{1} \partial_{i_{1}} \cdots_{1} \partial_{i_{l}}$. Hence, we can write the partie-finie integral in the form $\left(\forall N \in \mathbb{N}\right.$; with fixed $s^{\prime}$ such that $\left.0<s^{\prime}<1\right)$,

$$
\begin{aligned}
\int_{\mathbb{R}^{3} \backslash \mathcal{B}_{1}\left(s^{\prime}\right) \cup \mathcal{B}_{2}\left(s^{\prime}\right)} d^{3} \mathbf{x}_{\varepsilon} \delta_{1} F & +\frac{\varepsilon(1-\varepsilon)}{4 \pi} \sum_{a+\varepsilon \leqslant N} \frac{s^{\prime a+\varepsilon}}{a+\varepsilon} \int d \Omega_{1} f_{a} \\
& +\frac{\varepsilon(1-\varepsilon)}{4 \pi} \sum_{l \geqslant 0} \frac{(-)^{l}}{l !} \partial_{L} r_{12}^{\varepsilon-3}\left[\sum_{\substack{b+l+3 \leqslant N \\
\text { and } \neq 0}} \frac{s^{\prime b+l+3}}{b+l+3} \int d \Omega_{2} n_{2}^{L} f_{b}\right. \\
& \left.+\ln \left(\frac{s^{\prime}}{s_{2}}\right) \int d \Omega_{2} n_{2}^{L} f_{-l-3}\right]+o\left(s^{\prime N}\right) .
\end{aligned}
$$

Here, we have discarded the term with $\ln \left(s^{\prime} / s_{1}\right)$ by choosing $\varepsilon>0$ to be so small that all denominators $a+\varepsilon$ differ from zero. Since ${ }_{\varepsilon} \delta_{1}$ tends towards the Dirac measure when $\varepsilon \rightarrow 0$, the integral over $\mathbb{R}^{3} \backslash \mathcal{B}_{1}\left(s^{\prime}\right) \cup \mathcal{B}_{2}\left(s^{\prime}\right)$ goes to zero. Because of the factor $\varepsilon$ present in the numerators, so do the other terms when $\varepsilon \rightarrow 0$, except for those whose denominators involve a compensating $\varepsilon$. Now, the only term having the required property corresponds to $a=0$ in the previous expression. Therefore, taking the limit $\varepsilon \rightarrow 0$ (with fixed $s^{\prime}$ ), we get

$$
\lim _{\varepsilon \rightarrow 0} \operatorname{Pf} \int d^{3} \mathbf{x}_{\varepsilon} \delta_{1} F=\int \frac{d \Omega_{1}}{4 \pi} f_{0}\left(\mathbf{n}_{1}\right)+o\left(s^{\prime N}\right),
$$

and this being true for any $N$, we conclude

$$
\lim _{\varepsilon \rightarrow 0} \operatorname{Pf} \int d^{3} \mathbf{x}_{\varepsilon} \delta_{1} F=\int \frac{d \Omega_{1}}{4 \pi} f_{0}\left(\mathbf{n}_{1}\right)=(F)_{1}
$$

$(Q E D)$.

As we can infer from Lemma 2, the Riesz delta-function ${ }_{\varepsilon} \delta_{1}$ should constitute in the limit $\varepsilon \rightarrow 0$ an appropriate extension of the notion of Dirac distribution to the framework of parties finies of singular functions in $\mathcal{F}$. The precise definition of a "partie-finie Dirac function" necessitates the introduction of the space of linear forms on $\mathcal{F}$ and will be investigated in Sec. VI (see Definition 7).

\section{ALTERNATIVE FORMS OF THE PARTIE FINIE}

\section{A. Partie finie based on analytic continuation}

Practically speaking, the Hadamard partie-finie integral in the form given by (3.1) is rather difficult to evaluate, because it involves an integration over the complicated volume $\mathrm{R}^{3} \backslash \mathcal{B}_{1}(s) \cup \mathcal{B}_{2}(s)$. Fortunately, there exist several alternative expressions of the Hadamard partie 
finie, which are much better suited for practical computations. The first one is based on a double analytic continuation, with two complex parameters $\alpha, \beta \in \mathrm{C}$, of the integral

$$
I_{\alpha, \beta}=\int d^{3} \mathbf{x}\left(\frac{r_{1}}{s_{1}}\right)^{\alpha}\left(\frac{r_{2}}{s_{2}}\right)^{\beta} F
$$

where the constants $s_{1}$ and $s_{2}$ are the same as those introduced within the definition (3.1). The point for our purpose is that the integral (4.1) does range over the complete set $R^{3}$. First of all, we propose to check that $I_{\alpha, \beta}$ is defined by analytic continuation in a neighborhood of the origin $\alpha$ $=0=\beta$ in $\mathrm{C}_{1}^{2}$, except at the origin itself where it generically admits a simple pole in $\alpha$ or $\beta$ or both. We start by splitting $I_{\alpha, \beta}$ into three contribution: ${ }_{1} I_{\alpha, \beta}$ extending over the ball $\mathcal{B}_{1}(s)$ of radius $s$ surrounding $1,{ }_{2} I_{\alpha, \beta}$ extending over the ball $\mathcal{B}_{2}(s)$ surrounding 2 , and ${ }_{3} I_{\alpha, \beta}$ extending over the rest $\mathbb{R}^{3} \backslash \mathcal{B}_{1}(s) \cup \mathcal{B}_{2}(s)$. The integral ${ }_{1} I_{\alpha, \beta}$ is initially convergent for $\mathfrak{R}(\alpha)>-a_{0}-3$ and any $\beta$, where $a_{0}$ is the most singular power of $r_{1}$ in the expansion of $F$ near $\mathbf{y}_{1}$; similarly, ${ }_{2} I_{\alpha, \beta}$ exists only if $\mathfrak{R}(\beta)>-b_{0}-3$ and any $\alpha$ ( $b_{0}$ is the analogous to $a_{0}$ that relates to $\left.\mathbf{y}_{2}\right)$, and ${ }_{3} I_{\alpha, \beta}$ exists if $\mathfrak{R}(\alpha+\beta)<\epsilon$, where $\epsilon>0$ is such that $F=O\left(|\mathbf{x}|^{-3-\epsilon}\right)$ when $|\mathbf{x}| \rightarrow+\infty$. As the third contribution ${ }_{3} I_{\alpha, \beta}$ is clearly defined in a neighborhood of the origin, including the origin itself, we consider simply the part ${ }_{1} I_{\alpha, \beta}$ (the same reasoning applies $\operatorname{to}_{2} I_{\alpha, \beta}$ ). Within the integrand, we replace the product $r_{2}^{\beta} F$ by its expansion in the neighborhood of $\mathbf{y}_{1}$ (using a Taylor expansion for $r_{2}^{\beta}$ ), and find that the dependence on $\beta$ occurs through some everywhere well-defined quantity, namely ${ }_{1} \partial_{L} r_{12}^{\beta}$. After performing the angular integration over $d \Omega_{1}$, we obtain a remaining radial integral consisting of a sum of terms of the type $\int_{0}^{s} d r_{1} r_{1}^{\alpha+a+l+2}=s^{\alpha+a+l+3} /(\alpha+a+l+3)$, that clearly admit a unique analytic continuation on $\mathrm{CIZ}$; hence our statement (a simple pole at the origin arises when $a=-l-3$ ).

Theorem 2: For any function $F \in \mathcal{F}$ that is summable at infinity, the Hadamard partie finie of the integral is given by

$$
\mathrm{Pf}_{s_{1}, s_{2}} \int d^{3} \mathbf{x} F=\mathrm{FP}_{\substack{\alpha \rightarrow 0 \\ \beta \rightarrow 0}}^{\alpha} \int d^{3} \mathbf{x}\left(\frac{r_{1}}{s_{1}}\right)^{\alpha}\left(\frac{r_{2}}{s_{2}}\right)^{\beta} F=\mathrm{FP}_{\alpha \rightarrow 0}^{\beta \rightarrow 0} \int d^{3} \mathbf{x}\left(\frac{r_{1}}{s_{1}}\right)^{\alpha}\left(\frac{r_{2}}{s_{2}}\right)^{\beta} F
$$

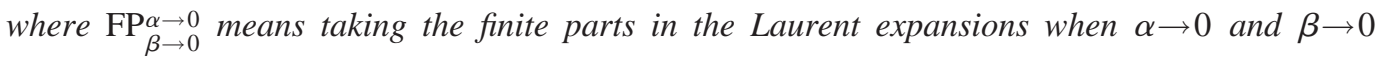
successively.

The proof of Theorem 2 is relegated to the Appendix. Notice our convention regarding the notation: while "Pf" always stands for the Partie finie of an integral in the specific sense of Hadamard, ${ }^{1}$ we refer to "FP" as the Finite Part or zeroth-order coefficient in the Laurent expansion with respect to some complex parameter $(\alpha, \beta \in \mathrm{C}$, or $B \in \mathrm{C}$ as in the next subsection). We see from Theorem 2 that the partie finie Pf can be viewed as a finite part FP and vice versa. The link between analytic continuation and Hadamard partie finie is pointed out by Schwartz. ${ }^{2}$ More precisely, Theorem 2 says how to calculate the Hadamard partie finie; the procedure consists of (i) performing the Laurent expansion of $I_{\alpha, \beta}$ when $\alpha \rightarrow 0$ while $\beta$ remains a fixed ("spectator") nonzero complex number, i.e.,

$$
I_{\alpha, \beta}=\sum_{p=p_{\min }}^{+\infty} \alpha^{p} I_{(p), \beta}
$$

where $p \in \mathbb{Z}$ and where the coefficients $I_{(p), \beta}$ depend on $\beta$; (ii) achieving the Laurent expansion of the zeroth- $\alpha$-power coefficient $I_{(0), \beta}$ when $\beta \rightarrow 0$, i.e.,

$$
I_{(0), \beta}=\sum_{q=q_{\min }}^{+\infty} \beta^{q} I_{(0, q)},
$$


to finally arrive at the zeroth- $\beta$-power coefficient $I_{(0,0)}$. Indeed, we find that the same result can be obtained by proceeding the other way around, first expanding around $\beta=0$ with a fixed $\alpha$, then expanding the coefficients $I_{\alpha,(0)}$ near $\alpha=0$. Thus,

$$
\mathrm{FP}_{\beta \rightarrow 0}\left\{\mathrm{FP}_{\alpha \rightarrow 0} I_{\alpha, \beta}\right\}=I_{(0,0)}=\mathrm{FP}_{\alpha \rightarrow 0}\left\{\mathrm{FP}_{\beta \rightarrow 0} I_{\alpha, \beta}\right\}
$$

We emphasize that the definition (3.1) of the partie finie yields unambiguously the result $I_{(0,0)}$, which corresponds to taking independently the two limits $\alpha \rightarrow 0$ and $\beta \rightarrow 0$ (the limiting process does not allow for instance to keep $\alpha=\beta)$. The final value $I_{(0,0)}$ is the same as the one given by the regularization adopted by Jaranowski and Schäfer ${ }^{12}$ (see their Appendix B.2).

In practice the expression (4.2) is used in connection with the Riesz formula, ${ }^{22}$ valid for any $\gamma, \delta \in \mathrm{C}$ except at some isolated poles,

$$
\int d^{3} \mathbf{x} r_{1}^{\gamma} r_{2}^{\delta}=\pi^{3 / 2} \frac{\Gamma\left(\frac{\gamma+3}{2}\right) \Gamma\left(\frac{\delta+3}{2}\right) \Gamma\left(-\frac{\gamma+\delta+3}{2}\right)}{\Gamma\left(-\frac{\gamma}{2}\right) \Gamma\left(-\frac{\delta}{2}\right) \Gamma\left(\frac{\gamma+\delta+6}{2}\right)} r_{12}^{\gamma+\delta+3}
$$

with $r_{12}=\left|\mathbf{y}_{1}-\mathbf{y}_{2}\right|$; here, $\Gamma$ denotes the Eulerian function. According to Theorem 2, the formula (4.4) permits computing the partie finie of any integral of a product between powers of $r_{1}$ and $r_{2}$. Consider the (not so trivial) case of the integral of $r_{1}^{-3} r_{2}^{-3}$, which is divergent at both points 1 and 2. From the Riesz formula, with $\gamma=\alpha-3$ and $\delta=\beta-3$, we have

$$
I_{\alpha, \beta}=\pi^{3 / 2} \frac{\Gamma\left(\frac{\alpha}{2}\right) \Gamma\left(\frac{\beta}{2}\right) \Gamma\left(-\frac{\alpha+\beta-3}{2}\right)}{\Gamma\left(-\frac{\alpha-3}{2}\right) \Gamma\left(-\frac{\beta-3}{2}\right) \Gamma\left(\frac{\alpha+\beta}{2}\right)} \frac{r_{12}^{\alpha+\beta-3}}{s_{1}^{\alpha} s_{2}^{\beta}}
$$

We compute the Laurent expansion when $\alpha \rightarrow 0$ with fixed $\beta \in \mathrm{C}$ and obtain a simple pole in $\alpha$ followed by a $\beta$-dependent finite part given by

$$
I_{(0), \beta}=\pi^{3 / 2} \frac{\Gamma(1)}{\Gamma\left(\frac{3}{2}\right)} \frac{r_{12}^{\beta-3}}{s_{2}^{\beta}}\left[\frac{2}{\beta}+\Psi(1)-\Psi\left(1+\frac{\beta}{2}\right)+\Psi\left(\frac{3}{2}\right)-\Psi\left(\frac{3}{2}-\frac{\beta}{2}\right)+2 \ln \left(\frac{r_{12}}{s_{1}}\right)\right],
$$

with $\Psi(z)=(d / d z) \ln \Gamma(z)$. This finite part itself includes a simple pole in $\beta$, and then we obtain the corresponding finite part when $\beta \rightarrow 0$ as

$$
I_{(0,0)}=\frac{\pi^{3 / 2}}{r_{12}^{3}} \frac{\Gamma(1)}{\Gamma\left(\frac{3}{2}\right)}\left[2 \ln \left(\frac{r_{12}}{s_{1}}\right)+2 \ln \left(\frac{r_{12}}{s_{2}}\right)\right]
$$

At last, Theorem 2 tells us that

$$
\mathrm{Pf}_{s_{1}, s_{2}} \int \frac{d^{3} \mathbf{x}}{r_{1}^{3} r_{2}^{3}}=\frac{4 \pi}{r_{12}^{3}}\left[\ln \left(\frac{r_{12}}{s_{1}}\right)+\ln \left(\frac{r_{12}}{s_{2}}\right)\right] .
$$

Some more complicated integrals will be obtained in the next subsection. 


\section{B. Partie finie based on angular integration}

The idea is to compute the partie-finie integral by performing an angular integration, followed by the integration over some radial variable. In a first stage, consider an integral that diverges at the point 1 , but converges at the point 2. According to (3.1), we need to compute it over the domain $\mathbb{R}^{3} \backslash \mathcal{B}_{1}(s)$; so it is natural to change the integration variable $\mathbf{x}$ to $\mathbf{r}_{1} \equiv \mathbf{x}-\mathbf{y}_{1}$, carry on the angular integration over $d \Omega_{1}=d \Omega\left(\mathbf{n}_{1}\right)$, and then, the radial integration over $r_{1}=\left|\mathbf{r}_{1}\right|$ varying from $s$ to infinity, i.e.,

$$
\int_{\mathbb{R}^{3} \backslash \mathcal{B}_{1}(s)} d^{3} \mathbf{x} F=\int_{r_{1}>s} d^{3} \mathbf{r}_{1} F=\int_{s}^{+\infty} d r_{1} r_{1}^{2} \int d \Omega_{1} F
$$

In the more general case where the integral is simultaneously divergent at the two points 1 and 2 , this method stricto sensu is no longer valid since the radial integration in (4.6) becomes divergent when $r_{1}=r_{12}$. Yet, still it is advantageous to dispose of a mean to change the variable $\mathbf{x}$ into $\mathbf{r}_{1}$ in order to obtain a convenient radial integration (even at the price of breaking the symmetry between the points 1 and 2). We shall derive here two Propositions, based on this idea, whose implementation in practical computations constitutes a very efficient mean to determine the partie finie, without any a priori restriction on the form of integrand as in the application of the Riesz formula (4.4).

As a matter of fact, in the first proposition, the computation of a partie-finie integral with two singularities 1 and 2 boils down to the computation of a partie-finie integral with singularity 1 and a finite-part integral (FP) whose singularity is located at infinity: $r_{1} \equiv\left|\mathbf{x}-\mathbf{y}_{1}\right| \rightarrow+\infty$ (so to speak, the singularity 2 is "rejected" to infinity).

Proposition 1: For any function $F$ in the class $\mathcal{F}$ we can write:

$$
\mathrm{Pf}_{s_{1}, s_{2}} \int d^{3} \mathbf{x} F=\mathrm{Pf}_{s_{1}}\left\{\operatorname{FP}_{B \rightarrow 0} \int d^{3} \mathbf{r}_{1}\left(\frac{r_{1}}{s_{2}}\right)^{B}\left[F-\sum_{b+3 \leqslant 0} r_{2}^{b} f_{b}\right]\right\}
$$

where the ${ }_{2} f_{b}$ 's denote the coefficients of the expansion of $F$ near $r_{2}=0$.

In other words, in order to compute the partie finie one can (i) "regularize" $F$ around the point 2 by subtracting out from it the terms yielding a divergence at 2, i.e.,

$$
\widetilde{F}_{2} \equiv F-\sum_{b+3 \leqslant 0} r_{2}^{b} f_{b}
$$

and (ii) compute the integral of the regularized $\widetilde{F}_{2}$ using the partie finie around 1 and the finite part when $B \rightarrow 0$ to deal with the divergency at infinity. Notice that the latter divergency has been introduced simply because of the term corresponding to $b=-3$ in (4.8) if nonzero. By the finite part when $B \rightarrow 0$ we mean the zeroth-order coefficient in the Laurent expansion of the analytic continuation with respect to the parameter $B \in \mathbb{C}$. The analytic continuation is straightforwardly defined from the domain of the complex plane $\mathfrak{R}(B)>0$ in which the integral converges at infinity.

Proof: We consider two open domains $\mathcal{D}_{1}$ and $\mathcal{D}_{2}$ that are supposed to be disjoined, $\mathcal{D}_{1} \cap \mathcal{D}_{2}=\varnothing$, complementary in $\mathbb{R}^{3}$, i.e. $\overline{\mathcal{D}_{1} \cup \mathcal{D}_{2}}=\mathbb{R}^{3}$, and such that $\mathbf{y}_{1} \in \mathcal{D}_{1}$ and $\mathbf{y}_{2} \in \mathcal{D}_{2}$. From Definition 4 , the partie-finie integral over $\mathcal{D}_{2}$ reads as (for small enough $s$ )

$$
\operatorname{Pf} \int_{\mathcal{D}_{2}} d^{3} \mathbf{x} F=\lim _{s \rightarrow 0}\left\{\int_{\mathcal{D}_{2} \backslash \mathcal{B}_{2}(s)} d^{3} \mathbf{x} F+\sum_{b+3<0} \frac{s^{b+3}}{b+3} \int d \Omega_{2} f_{b}+\ln \left(\frac{s}{s_{2}}\right) \int d \Omega_{2} f_{-3}\right\} .
$$

Now, two short computations reveal that 


$$
\begin{gathered}
\sum_{b+3<0} \int_{\mathbb{R}^{3} \backslash \mathcal{B}_{2}(s)} d^{3} \mathbf{x} r_{2}^{b} f_{b}=-\sum_{b+3<0} \frac{s^{b+3}}{b+3} \int d \Omega_{2} f_{b}, \\
\mathrm{FP}_{B \rightarrow 0} \int_{\mathbb{R}^{3} \backslash \mathcal{B}_{2}(s)} d^{3} \mathbf{x}\left(\frac{|\mathbf{x}|}{s_{2}}\right)^{B} \frac{1}{r_{2}^{3}} f_{-3}=-\ln \left(\frac{s}{s_{2}}\right) \int d \Omega_{2} f_{-3} .
\end{gathered}
$$

Furthermore, since the integral appearing in (4.9a) is convergent at infinity, one can add without harm the same finite part operation when $B \rightarrow 0$ as in (4.9b). Thus, the integral over $\mathcal{D}_{2}$ may be re-written as

$$
\begin{aligned}
\lim _{s \rightarrow 0}\left\{\int_{\mathcal{D}_{2} \backslash \mathcal{B}_{2}(s)} d^{3} \mathbf{x} F-\sum_{b+3 \leqslant 0} \mathrm{FP}_{B \rightarrow 0} \int_{\mathrm{R}^{3} \backslash \mathcal{B}_{2}(s)} d^{3} \mathbf{x}\left(\frac{|\mathbf{x}|}{s_{2}}\right)^{B} r_{2}^{b} f_{b}\right\} \\
\quad=\lim _{s \rightarrow 0}\left\{\mathrm{FP}_{B \rightarrow 0} \int_{\mathcal{D}_{2} \backslash \mathcal{B}_{2}(s)} d^{3} \mathbf{x}\left(\frac{|\mathbf{x}|}{s_{2}}\right)^{B} \widetilde{F}_{2}-\sum_{b+3 \leqslant 0} \mathrm{FP}_{B \rightarrow 0} \int_{\mathcal{D}_{1}} d^{3} \mathbf{x}\left(\frac{|\mathbf{x}|}{s_{2}}\right)^{B} r_{2}^{b} f_{b}\right\} \\
\quad=\lim _{s \rightarrow 0}\left\{\operatorname{FP}_{B \rightarrow 0} \int_{\mathcal{D}_{2}} d^{3} \mathbf{x}\left(\frac{|\mathbf{x}|}{s_{2}}\right)^{B} \widetilde{F}_{2}-\sum_{b+3 \leqslant 0} \operatorname{FP}_{B \rightarrow 0} \int_{\mathcal{D}_{1} \backslash \mathcal{B}_{1}(s)} d^{3} \mathbf{x}\left(\frac{|\mathbf{x}|}{s_{2}}\right)^{B} r_{2}^{b} f_{b}\right\} .
\end{aligned}
$$

We have used the facts that the integral of $F$ converges at infinity (first equality) and the integral of $\widetilde{F}_{2}$ converges at the singularity 2 (second equality). Adding up the other contribution extending over $\mathcal{D}_{1}$, we readily obtain the complete partie finie as

$$
\lim _{s \rightarrow 0}\left\{\operatorname{FP}_{B \rightarrow 0} \int_{\mathbb{R}^{3} \backslash \mathcal{B}_{1}(s)} d^{3} \mathbf{x}\left(\frac{|\mathbf{x}|}{s_{2}}\right)^{B} \widetilde{F}_{2}+\sum_{a+3<0} \frac{s^{a+3}}{a+3} \int d \Omega_{1} f_{a}+\ln \left(\frac{s}{s_{1}}\right) \int d \Omega_{1} f_{-3}\right\} .
$$

Since the coefficients ${ }_{1} f_{a}$, for $a \leqslant-3$, are those of the expansion when $r_{1} \rightarrow 0$ of $F$ as well as of $\widetilde{F}_{2}$, we recognize in the expression above the partie finie (with respect to 1 only) of the integral of the regularized function $\widetilde{F}_{2}$. Hence the intermediate expression

$$
\mathrm{Pf}_{s_{1}, s_{2}} \int d^{3} \mathbf{x} F=\mathrm{Pf}_{s_{1}}\left\{\operatorname{FP}_{B \rightarrow 0} \int d^{3} \mathbf{x}\left(\frac{|\mathbf{x}|}{s_{2}}\right)^{B} \widetilde{F}_{2}\right\} .
$$

To establish the proposition it remains to change variable $\mathbf{x}$ into $\mathbf{r}_{1}$. At that point, we must be careful, because under this change of variable the regularization factor $|\mathbf{x}|^{B}$ changes itself in a complicated way. Fortunately, we can limit ourselves to the case where $B$ is infinitesimal, since we shall take the finite part afterwards, making $B \rightarrow 0$. We substitute to $|\mathbf{x}|^{B}$ in the right side of (4.10) its equivalent expression in terms of $\mathbf{r}_{1}$ and where we expand when $B \rightarrow 0$, i.e.,

$$
|\mathbf{x}|^{B}=r_{1}^{B} e^{B \ln \left(|\mathbf{x}| / r_{1}\right)}=r_{1}^{B}\left\{1+\frac{B}{2} \ln \left[1+2 \frac{\mathbf{n}_{1} \cdot \mathbf{y}_{1}}{r_{1}}+\frac{\mathbf{y}_{1}^{2}}{r_{1}^{2}}\right]+O\left(B^{2}\right)\right\},
$$

where $\mathbf{n}_{1} \cdot \mathbf{y}_{1}$ denotes the usual scalar product on $\mathbb{R}^{3}$ (and $\mathbf{y}_{1}^{2}=\mathbf{y}_{1} \cdot \mathbf{y}_{1}$ ). Now, the dominant term in the latter expansion amounts simply to replacing $|\mathbf{x}|^{B}$ by $r_{1}^{B}$, which would yield precisely the result (4.7) we want to prove; but we have still to show that all the extra terms in the expansion (4.11), which carry at least a factor $B$ in front, do not contribute to the final result, i.e., that

$$
\mathrm{FP}_{B \rightarrow 0}\left[\frac{B}{2} \int^{+\infty} d^{3} \mathbf{r}_{1}\left(\frac{r_{1}}{s_{2}}\right)^{B} \ln \left[1+2 \frac{\mathbf{n}_{1} \cdot \mathbf{y}_{1}}{r_{1}}+\frac{\mathbf{y}_{1}^{2}}{r_{1}^{2}}\right] \widetilde{F}_{2}+O\left(B^{2}\right)\right]=0 .
$$

Because of the factor $B$ in front, the only possible contribution to the finite part for $B \rightarrow 0$ occurs when the integral develops a pole at $B=0$ due to the behavior of the integrand at infinity $\left(r_{1} \rightarrow\right.$ 
$+\infty)$. Hence, as indicated in (4.12), the value of the integral depends only on the bound at infinity [this is also why we did not write a $\mathrm{Pf}_{s_{1}}$ symbol in (4.12): the partie finie deals with the bound $r_{1}=0$, which is irrelevant to this case]. In order to evaluate the pole, we replace the integrand by its expansion when $r_{1} \rightarrow+\infty$. We know that $F$ behaves as $o\left(1 /|\mathbf{x}|^{3}\right)$ at a maximum $|\mathbf{x}| \rightarrow+\infty$ to ensure the convergence of the integral of $F$ at infinity, so we have $F=o\left(1 / r_{1}^{3}\right)$ when $r_{1} \rightarrow+\infty$. Now, from the defining expression (4.8) of $\widetilde{F}_{2}$, we obtain

$$
\widetilde{F}_{2}=-\frac{1}{r_{12}^{3}} f_{-3}\left(\mathbf{n}_{1}\right)+o\left(\frac{1}{r_{1}^{3}}\right), \quad \text { when } r_{1} \rightarrow+\infty
$$

after making the replacements of $r_{2}$ and $\mathbf{n}_{2}$ by $r_{1}$ and $\mathbf{n}_{1}$ which are permitted because we are working at the dominant order when $r_{1} \rightarrow+\infty$. On the other hand, we have $\ln \left[1+2\left[\left(\mathbf{n}_{1} \cdot \mathbf{y}_{1}\right) / r_{1}\right]\right.$ $\left.\left.+\mathbf{y}_{1}^{2} / r_{1}^{2}\right]=2\left[\left(\mathbf{n}_{1} \cdot \mathbf{y}_{1}\right) / r_{1}\right]+O 1 / r_{1}^{2}\right)$. So that the integral to be computed (as concerns the only relevant bound at infinity) reads as

$$
\int^{+\infty} d^{3} \mathbf{r}_{1} r_{1}^{B} \ln \left[1+2 \frac{\mathbf{n}_{1} \cdot \mathbf{y}_{1}}{r_{1}}+\frac{\mathbf{y}_{1}^{2}}{r_{1}^{2}}\right] \widetilde{F}_{2}=-2 \int^{+\infty} d r_{1} r_{1}^{B-2}\left\{\int d \Omega_{1} \mathbf{n}_{1} \cdot \mathbf{y}_{1} f_{-3}\left(\mathbf{n}_{1}\right)+o\left(r_{1}^{0}\right)\right\} .
$$

This integral cannot generate a pole at $B=0$ since such a pole could come only from a radial integral of the type $\int^{+\infty} d r_{1} r_{1}^{B-1}$ (after the angular integration has been performed). Repeating the same reasoning to any higher orders in $B$, we prove the equation (4.12) as well as Proposition 1.

In practice, Proposition 1 is used with the integration with respect to $\mathbf{n}_{1}$, followed by the integration over $r_{1}$ varying from $0\left(\mathrm{Pf}_{s_{1}}\right.$ takes care of this bound) to infinity (where $\mathrm{FP}_{B \rightarrow 0}$ does the work); Proposition 1 justifies this process even when the original integral is divergent at both singularities. The result of the angular integration depends on where the field point is located, either inside the ball $\mathcal{B}_{1}\left(r_{12}\right)$ centered on $\mathbf{y}_{1}$ and of radius $r_{12}$ (the point 2 lies on the surface of this ball), or in the complementary domain $\mathbb{R}^{3} \backslash \mathcal{B}_{1}\left(r_{12}\right)$. Therefore, a natural splitting of the integral (4.7) is

$$
\mathrm{Pf}_{s_{1}, s_{2}} \int d^{3} \mathbf{x} F=\mathrm{Pf}_{s_{1}} \int_{\mathcal{B}_{1}\left(r_{12}\right)} d^{3} \mathbf{r}_{1} \widetilde{F}_{2}+\mathrm{FP}_{B \rightarrow 0} \int_{\mathbb{R}^{3} \backslash \mathcal{B}_{1}\left(r_{12}\right)} d^{3} \mathbf{r}_{1}\left(\frac{r_{1}}{s_{2}}\right)^{B} \widetilde{F}_{2},
$$

taking into account the fact that the partie finie $\mathrm{Pf}_{s_{1}}$ applies only to the inner integral, over $\mathcal{B}_{1}\left(r_{12}\right)$, and the finite part $\mathrm{FP}_{B \rightarrow 0}$ only to the outer one, over $\mathbb{R}^{3} \backslash \mathcal{B}_{1}\left(r_{12}\right)$. To be more specific, the angular integral of $\widetilde{F}_{2}$ defines two angular-average functions $\widetilde{I}_{2}\left(r_{1}\right)$ and $\widetilde{J}_{2}\left(r_{1}\right)$ depending on whether $\mathbf{x}$ is in $\mathcal{B}_{1}\left(r_{12}\right)$ or its complement:

$$
\int \frac{d \Omega_{1}}{4 \pi} \widetilde{F}_{2}=\left\{\begin{array}{ccc}
\widetilde{I}_{2}\left(r_{1}\right), & \text { when } & r_{1} \leqslant r_{12}, \\
\widetilde{J}_{2}\left(r_{1}\right), & \text { when } & r_{1}>r_{12} .
\end{array}\right.
$$

The functions $\widetilde{I}_{2}$ and $\widetilde{J}_{2}$ depend also explicitly on the source points $\mathbf{y}_{1}$ and $\mathbf{y}_{2}$. [As an example, in the case $\widetilde{F}_{2}=r_{2}$, we find $\widetilde{I}_{2}=r_{12}+r_{1}^{2} / 3 r_{12}$ and $\widetilde{J}_{2}=r_{1}+r_{12}^{2} / 3 r_{1}$.] Now, knowing $\widetilde{I}_{2}$ and $\widetilde{J}_{2}$, we can achieve the radial integration according to the formula

$$
\mathrm{Pf}_{s_{1}, s_{2}} \int d^{3} \mathbf{x} F=4 \pi \mathrm{Pf}_{s_{1}} \int_{0}^{r_{12}} d r_{1} r_{1}^{2} \widetilde{I}_{2}+4 \pi \mathrm{FP}_{B \rightarrow 0} \int_{r_{12}}^{+\infty} d r_{1}\left(\frac{r_{1}}{s_{2}}\right)^{B} r_{1}^{2} \widetilde{J}_{2} .
$$

The first term in (4.16) is quite simple to handle in practice, whereas the second one is more difficult because it requires a priori the knowledge of a closed-form expression for the integral of $r_{1}^{B+2} \widetilde{J}_{2}$, valid for any $B$ such that $\mathfrak{R}(B)>0$. Obtaining this may not be feasible if $F$ is too 
complicated; in this event, we should use a different form of the integral at infinity. The second proposition, which provides the appropriate form, constitutes, perhaps, the most powerful way to compute the partie finie in rather complicated applications.

Proposition 2: The partie finie of the integral of $F \in \mathcal{F}$ (if convergent at infinity) reads as:

$$
\begin{aligned}
\mathrm{Pf}_{s_{1}, s_{2}} \int d^{3} \mathbf{x} F= & 4 \pi \mathrm{Pf}_{s_{1}} \int_{0}^{r_{12}} d r_{1} r_{1}^{2} \widetilde{I}_{2}\left(r_{1}\right)+4 \pi \int_{r_{12}}^{+\infty} d r_{1}\left[r_{1}^{2} \widetilde{J}_{2}\left(r_{1}\right)+\frac{1}{r_{1}}\left(r_{2}^{3} F\right)_{2}\right] \\
& +4 \pi\left(r_{2}^{3} F\right)_{2} \ln \left(\frac{r_{12}}{s_{2}}\right)
\end{aligned}
$$

(and similarly by interchange of 1 and 2).

Proof: Consider the angular average of the expansion of $\widetilde{F}_{2}$ when $r_{1} \rightarrow+\infty$ which has been determined in (4.13). We get

$$
\widetilde{J}_{2} \equiv \int \frac{d \Omega_{1}}{4 \pi} \widetilde{F}_{2}=-\frac{1}{r_{1}^{3}}\left(r_{2}^{3} F\right)_{2}+o\left(\frac{1}{r_{1}^{3}}\right)
$$

where the coefficient of the dominant term is made of a Hadamard partie finie at point 2. Let us subtract and add to $\widetilde{J}_{2}$ inside the second integral in (4.16) the previous dominant term at infinity. In this way, we may re-write it as the sum of a convergent integral at infinity on one hand, to which we can then remove the finite part prescription, and a simple extra integral on the other hand. Namely,

$$
\int_{r_{12}}^{+\infty} d r_{1}\left[r_{1}^{2} \widetilde{J}_{2}+\frac{1}{r_{1}}\left(r_{2}^{3} F\right)_{2}\right]-\left(r_{2}^{3} F\right)_{2} \mathrm{FP}_{B \rightarrow 0} \int_{r_{12}}^{+\infty} \frac{d r_{1}}{r_{1}}\left(\frac{r_{1}}{s_{2}}\right)^{B}
$$

The extra integral is finally computed in a simple way as

$$
\mathrm{FP}_{B \rightarrow 0} \int_{r_{12}}^{+\infty} \frac{d r_{1}}{r_{1}}\left(\frac{r_{1}}{s_{2}}\right)^{B}=\mathrm{FP}_{B \rightarrow 0}\left[-\frac{1}{B}\left(\frac{r_{12}}{s_{2}}\right)^{B}\right]=-\ln \left(\frac{r_{12}}{s_{2}}\right)
$$

where we used the properties of the analytic continuation.

QED.

Thanks to Proposition 2 we are now able to compute many integrals which could not be deduced from the Riesz formula (4.4), unlike for (4.5). For instance,

$$
\begin{gathered}
\mathrm{Pf}_{s_{1}, s_{2}} \int \frac{d^{3} \mathbf{x}}{r_{1}^{3} r_{2}^{3}\left(r_{1}+r_{2}\right)}=\frac{4 \pi}{r_{12}^{4}}\left[\ln \left(\frac{r_{12}}{s_{1}}\right)+\ln \left(\frac{r_{12}}{s_{2}}\right)-\frac{8}{3} \ln 2+\frac{2}{3}\right], \\
\mathrm{Pf}_{s_{1}, s_{2}} \int \frac{d^{3} \mathbf{x}}{r_{1}^{3} r_{2}^{3}\left(r_{1}+r_{2}+r_{12}\right)}=\frac{2 \pi}{r_{12}^{4}}\left[\ln \left(\frac{r_{12}}{s_{1}}\right)+\ln \left(\frac{r_{12}}{s_{2}}\right)+\frac{\pi^{2}}{3}-4\right] .
\end{gathered}
$$

The result for the integral $(4.19 \mathrm{~b})$ is in agreement with the one that follows from a recent generalization of the Riesz formula to include arbitrary powers of $r_{1}+r_{2}+r_{12}$, which has been obtained by Jaranowski and Schäfer (see Appendix B.2 in Ref. 12). In any case, the dependence of the partie-finie integral on the two constants $s_{1}$ and $s_{2}$ is given by

$$
\mathrm{Pf}_{s_{1}, s_{2}} \int d^{3} \mathbf{x} F=4 \pi\left(r_{1}^{3} F\right)_{1} \ln \left(\frac{r_{12}}{s_{1}}\right)+4 \pi\left(r_{2}^{3} F\right)_{2} \ln \left(\frac{r_{12}}{s_{2}}\right)+\text { terms independent of } s_{1}, s_{2} \text {. }
$$




\section{PARTIE FINIE OF POISSON INTEGRALS}

In this section we investigate the main properties of the partie finie of Poisson integrals of singular functions in the class $\mathcal{F}$. We have in view the application to the post-Newtonian motion of particles in general relativity, since the post-Newtonian iteration proceeds typically through Poisson (or Poisson-type) integrals. Consider a fixed ("spectator") point $\mathbf{x}^{\prime} \in \mathbb{R}^{3}$ and, for each value of $\mathbf{x}^{\prime}$, define the function $S_{\mathbf{x}^{\prime}}(\mathbf{x})=F(\mathbf{x}) /\left|\mathbf{x}-\mathbf{x}^{\prime}\right|$ where $F \in \mathcal{F}$. Clearly, for any given $\mathbf{x}^{\prime}$, the function $S_{\mathbf{x}^{\prime}}$ belongs to the class $\mathcal{F}_{\text {loc }}$, introduced in Sec. II, Definition 2. In addition, when the spectator point $\mathbf{x}^{\prime}$ coincides with the singular point $\mathbf{y}_{1}$ (and similarly for $\mathbf{y}_{2}$ ), we have $S_{\mathbf{y}_{1}} \in \mathcal{F}$. Since (as already mentioned) Definition 4 can be extended to functions in the class $\mathcal{F}_{\text {loc }}$, we can consider the partie-finie integral

$$
P\left(\mathbf{x}^{\prime}\right)=-\frac{1}{4 \pi} \operatorname{Pf} \int d^{3} \mathbf{x} S_{\mathbf{x}^{\prime}}(\mathbf{x})=-\frac{1}{4 \pi} \operatorname{Pf} \int \frac{d^{3} \mathbf{x}}{\left|\mathbf{x}-\mathbf{x}^{\prime}\right|} F(\mathbf{x}) .
$$

This is, indeed, what we shall call the "Poisson" integral of $F$. In particular, when the spectator point $\mathbf{x}^{\prime}$ is equal to $\mathbf{y}_{1}$, we shall write

$$
P\left(\mathbf{y}_{1}\right)=-\frac{1}{4 \pi} \operatorname{Pf} \int d^{3} \mathbf{x} S_{\mathbf{y}_{1}}(\mathbf{x})=-\frac{1}{4 \pi} \operatorname{Pf} \int \frac{d^{3} \mathbf{x}}{r_{1}} F(\mathbf{x}) .
$$

The Poisson integral is not continuous at the singular point $\mathbf{y}_{1}$ because $P\left(\mathbf{x}^{\prime}\right)$, when initially defined for $\mathbf{x}^{\prime} \neq=\mathbf{y}_{1}$, admits an expansion that is singular when $\mathbf{x}^{\prime}$ tends to $\mathbf{y}_{1}$. In the present Section, our aim is to understand the limit relation of the integral $P\left(\mathbf{x}^{\prime}\right)$ when $r_{1}^{\prime} \equiv\left|\mathbf{x}^{\prime}-\mathbf{y}_{1}\right| \rightarrow 0$, and to connect it with the "regularized" integral $P\left(\mathbf{y}_{1}\right)$ given by (5.2). In particular, we shall show that the "partie finie" (in an extended Hadamard's sense) of $P\left(\mathbf{x}^{\prime}\right)$ at $\mathbf{x}^{\prime}=\mathbf{y}_{1}$ is related in a precise way to $P\left(\mathbf{y}_{1}\right)$. Let us make clear straight away that $P\left(\mathbf{x}^{\prime}\right)$, as a function of $\mathbf{x}^{\prime}$ different from $\mathbf{y}_{1}$ (and $\mathbf{y}_{2}$ ), does not belong to the class $\mathcal{F}$ as the Poisson integral typically generates logarithms in the expansion when $r_{1}^{\prime} \rightarrow 0$. In particular, the coefficient of zeroth power of $r_{1}^{\prime}$ in the latter expansion contains a priori a $\ln r_{1}^{\prime}$ term, and its partie finie in the sense of Definition 3 is in fact not finite at all, because of the presence of this formally infinite constant $\ln r_{1}^{\prime}=-\infty$. A possible way to deal with this problem, followed by Sellier in Ref. 5, is to exclude the $\ln r_{1}^{\prime}$ (and any higher power of $\ln r_{1}^{\prime}$ ) from the definition of the partie finie. On the other hand, in applications to the physical problem, the constant $\ln r_{1}^{\prime}$ can be viewed as a "renormalization" constant, which is better to keep as it appears all the way through the calculation. Therefore, we simply include here the renormalization constant $\ln r_{1}^{\prime}$ into the definition; but, for simplicity's sake, we stick to the name of "partie finie" in this case (although the $\ln r_{1}^{\prime}$ makes it formally infinite). Thus, for a function like $P$ admitting a logarithmic expansion:

$$
\forall N \in \mathbb{N}, \quad P\left(\mathbf{x}^{\prime}\right)=\sum_{\substack{a \leqslant N \\ p=0,1}} r_{1}^{\prime a}\left(\ln r_{1}^{\prime}\right)^{p} f_{a, p}\left(\mathbf{n}_{1}^{\prime}\right)+o\left(r_{1}^{\prime N}\right), \text { when } r_{1}^{\prime} \rightarrow 0,
$$

we define the Hadamard partie finie of $P$ at 1 by

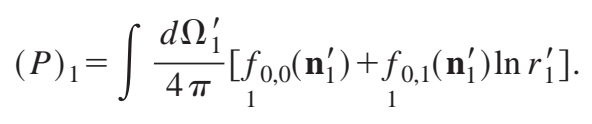

Theorem 3: The Hadamard partie finie at 1 (in the previous sense) of the Poisson integral of any $F \in \mathcal{F}$ reads as

$$
(P)_{1}=-\frac{1}{4 \pi} \operatorname{Pf}_{s_{1}, s_{2}} \int \frac{d^{3} \mathbf{x}}{r_{1}} F(\mathbf{x})+\left[\ln \left(\frac{r_{1}^{\prime}}{s_{1}}\right)-1\right]\left(r_{1}^{2} F\right)_{1},
$$


with $r_{1}^{\prime}=\left|\mathbf{x}^{\prime}-\mathbf{y}_{1}\right|$. Furthermore the constants $s_{1}$ cancel each other from the two terms in the right side of (5.5) (so the partie finie depends on the two constants $\ln r_{1}^{\prime}$ and $\ln s_{2}$ ).

In other words, the partie finie of the Poisson integral at 1 is equal to the regularized integral $P\left(\mathbf{y}_{1}\right)$, obtained from the replacement $\mathbf{x}^{\prime} \rightarrow \mathbf{y}_{1}$ inside the integrand of $P\left(\mathbf{x}^{\prime}\right)$, augmented by a term associated with the presence of the (infinite) constant $\ln r_{1}^{\prime}$.

Proof: The fact that the constants $s_{1}$ cancel out (so $s_{1}$ is "replaced" by $r_{1}^{\prime}$ ) is a trivial consequence of the dependence of the partie finie on $s_{1}$ and $s_{2}$ determined in (4.20). For our proof, we need the explicit expressions of the objects $P\left(\mathbf{x}^{\prime}\right)$, when $\mathbf{x}^{\prime}$ is different from $\mathbf{y}_{1}$ and $\mathbf{y}_{2}$, and $P\left(\mathbf{y}_{1}\right)$, following from Definition 4. For $\mathbf{x}^{\prime} \neq \mathbf{y}_{1}$ and $r_{1}=\left|\mathbf{x}-\mathbf{y}_{1}\right| \rightarrow 0$, we have the expansion

$$
S_{\mathbf{x}^{\prime}}(\mathbf{x})=\sum_{l \geqslant 0} \frac{(-)^{l}}{l !} \partial_{L}^{\prime}\left(\frac{1}{r_{1}^{\prime}}\right) \sum_{a} r_{1}^{a+l} n_{1}^{L} f_{a}\left(\mathbf{n}_{1}\right)
$$

(and idem $1 \leftrightarrow 2$ ), where $r_{1}^{\prime}=\left|\mathbf{x}^{\prime}-\mathbf{y}_{1}\right|, \partial_{L}^{\prime}$ being the multi-spatial derivative acting on $\mathbf{x}^{\prime}$. From (3.1), we get the expression (for $\mathbf{x}^{\prime} \neq \mathbf{y}_{1}$ and $\mathbf{y}_{2}$ )

$$
\begin{aligned}
P\left(\mathbf{x}^{\prime}\right)= & -\frac{1}{4 \pi} \lim _{s \rightarrow 0}\left\{\int_{\mathbb{R}^{3} \backslash \mathcal{B}_{1}(s) \cup \mathcal{B}_{2}(s)} \frac{d^{3} \mathbf{x}}{\left|\mathbf{x}-\mathbf{x}^{\prime}\right|} F\right. \\
& +\sum_{l \geqslant 0} \frac{(-)^{l}}{l !} \partial_{L}^{\prime}\left(\frac{1}{r_{1}^{\prime}}\right)\left[\sum_{a+l+3<0} \frac{s^{a+l+3}}{a+l+3} \int d \Omega_{1} n_{1}^{L} f_{a}\right. \\
& \left.\left.+\ln \left(\frac{s}{s_{1}}\right) \int d \Omega_{1} n_{1}^{L} f_{-3-l}\right]+1 \leftrightarrow 2\right\} .
\end{aligned}
$$

Applying the recipe (5.4), we start by computing the angular integral over $\mathbf{n}_{1}^{\prime}=\left(\mathbf{x}^{\prime}-\mathbf{y}_{1}\right) / r_{1}^{\prime}$ (for a fixed $\left.r_{1}^{\prime}\right)$ of $P\left(\mathbf{x}^{\prime}\right)$ in the form given by (5.7), and consider the limit $r_{1}^{\prime} \rightarrow 0$ afterwards. Since $s$ is fated to tend to zero first, one can choose $s<r_{1}^{\prime}$, and as we are ultimately interested in the limit $r_{1}^{\prime} \rightarrow 0$, we also assume $r_{1}^{\prime}<r_{12}$. To compute the angular average of the divergent terms in (5.7), we make use of the identities

$$
\begin{gathered}
\int \frac{d \Omega_{1}^{\prime}}{4 \pi} \partial_{L}^{\prime}\left(\frac{1}{r_{1}^{\prime}}\right)=\frac{\delta_{0 l}}{r_{1}^{\prime}}, \\
\int \frac{d \Omega_{1}^{\prime}}{4 \pi} \partial_{L}^{\prime}\left(\frac{1}{r_{2}^{\prime}}\right)=\partial_{L}\left(\frac{1}{r_{12}}\right)
\end{gathered}
$$

(where $\delta_{0 l}$ denotes the Kronecker symbol). On the other hand, the relevant formula to treat the integral on the right side of (5.7) is

$$
\int \frac{d \Omega_{1}^{\prime}}{4 \pi} \frac{1}{\left|\mathbf{x}-\mathbf{x}^{\prime}\right|}= \begin{cases}\frac{1}{r_{1}^{\prime}} & \left(\text { if } r_{1}<r_{1}^{\prime}\right), \\ \frac{1}{r_{1}} & \left(\text { if } r_{1}^{\prime}<r_{1}\right) .\end{cases}
$$

We split this integral into three other ones, the first of them extending over the "exterior" domain $\mathrm{R}^{3} \backslash \mathcal{B}_{1}\left(r_{1}^{\prime}\right) \cup \mathcal{B}_{2}\left(r_{1}^{\prime}\right)$, and the two remaining ones over the ring-shaped regions $\mathcal{B}_{1}\left(r_{1}^{\prime}\right) \backslash \mathcal{B}_{1}(s)$ and $\mathcal{B}_{2}\left(r_{1}^{\prime}\right) \backslash \mathcal{B}_{2}(s)$. Hence 


$$
\begin{aligned}
\int \frac{d \Omega_{1}^{\prime}}{4 \pi} P\left(\mathbf{x}^{\prime}\right)= & -\frac{1}{4 \pi} \lim _{s \rightarrow 0}\left\{\int_{\mathbb{R}^{3} \backslash \mathcal{B}_{1}\left(r_{1}^{\prime}\right) \cup \mathcal{B}_{2}\left(r_{1}^{\prime}\right)} \frac{d^{3} \mathbf{x}}{r_{1}} F+\frac{1}{r_{1}^{\prime}} \int_{\mathcal{B}_{1}\left(r_{1}^{\prime}\right) \backslash \mathcal{B}_{1}(s)} d^{3} \mathbf{x} F+\int_{\mathcal{B}_{2}\left(r_{1}^{\prime}\right) \backslash \mathcal{B}_{2}(s)} \frac{d^{3} \mathbf{x}}{r_{1}} F\right. \\
& +\frac{1}{r_{1}^{\prime}}\left[\sum_{a+3<0} \frac{s^{a+3}}{a+3} \int d \Omega_{1} f_{a}+\ln \left(\frac{s}{s_{1}}\right) \int d \Omega_{1} f_{-3}\right]+\sum_{l \geqslant 0} \frac{(-)^{l}}{l !} \partial_{L}\left(\frac{1}{r_{12}}\right) \\
& \left.\times\left[\sum_{b+l+3<0} \frac{s^{b+l+3}}{b+l+3} \int d \Omega_{2} n_{2}^{L} f_{b}+\ln \left(\frac{s}{s_{2}}\right) \int d \Omega_{2} n_{2}^{L} f_{-3-l}\right]\right\}
\end{aligned}
$$

Next, supposing that $r_{1}^{\prime}$ is small enough, we may replace $F$ in the second and third terms by its own expansions around 1 and 2, respectively. We find that the divergent terms in $s$ cancel out, so we are allowed to apply the limit $s \rightarrow 0$. This yields

$$
\begin{aligned}
\int \frac{d \Omega_{1}^{\prime}}{4 \pi} P\left(\mathbf{x}^{\prime}\right)= & -\frac{1}{4 \pi}\left\{\int_{\mathbb{R}^{3} \backslash \mathcal{B}_{1}\left(r_{1}^{\prime}\right) \cup \mathcal{B}_{2}\left(r_{1}^{\prime}\right)} \frac{d^{3} \mathbf{x}}{r_{1}} F\right. \\
& +\frac{1}{r_{1}^{\prime}}\left[\sum_{a+3<0} \frac{r_{1}^{\prime a+3}}{a+3} \int d \Omega_{1} f_{a}+\ln \left(\frac{r_{1}^{\prime}}{s_{1}}\right) \int d \Omega_{1} f_{-3}+r_{1}^{\prime} \int d \Omega_{1} f_{-2}\right] \\
& +\sum_{l \geqslant 0} \frac{(-)^{l}}{l !} \partial_{L}\left(\frac{1}{r_{12}}\right)\left[\sum_{b+l+3<0} \frac{r_{1}^{\prime b+l+3}}{b+l+3} \int d \Omega_{2} n_{2}^{L} f_{b}+\ln \left(\frac{r_{1}^{\prime}}{s_{2}}\right) \int d \Omega_{2} n_{2}^{L} f_{-3-l}\right] \\
& \left.+o\left(r_{1}^{\prime 0}\right)\right\}
\end{aligned}
$$

(the remainder dies out when $r_{1}^{\prime} \rightarrow 0$ ). Under the latter form we recognize most of the terms composing the integral $P\left(\mathbf{y}_{1}\right)$. Indeed, we have, respectively, when $r_{1} \rightarrow 0$ and $r_{2} \rightarrow 0$,

$$
\begin{gathered}
S_{\mathbf{y}_{1}}(\mathbf{x})=\sum_{a} r_{1}^{a-1} f_{a}\left(\mathbf{n}_{1}\right), \\
S_{\mathbf{y}_{1}}(\mathbf{x})=\sum_{l \geqslant 0} \frac{(-)^{l}}{l !} \partial_{L}\left(\frac{1}{r_{12}}\right) \sum_{b} r_{2}^{b+l} n_{2}^{L} f_{b}\left(\mathbf{n}_{2}\right) .
\end{gathered}
$$

Now, using the form (3.3) of the partie finie with the change of notation $s^{\prime}=r_{1}^{\prime}$, we find

$$
\begin{aligned}
P\left(\mathbf{y}_{1}\right)= & -\frac{1}{4 \pi}\left\{\int_{\mathbb{R}^{3} \backslash \mathcal{B}_{1}\left(r_{1}^{\prime}\right) \cup \mathcal{B}_{2}\left(r_{1}^{\prime}\right)} \frac{d^{3} \mathbf{x}}{r_{1}} F+\sum_{a+2<0} \frac{r_{1}^{\prime a+2}}{a+2} \int d \Omega_{1} f_{a}+\ln \left(\frac{r_{1}^{\prime}}{s_{1}}\right) \int d \Omega_{1} f_{-2}\right. \\
& \left.+\sum_{l \geqslant 0} \frac{(-)^{l}}{l !} \partial_{L}\left(\frac{1}{r_{12}}\right)\left[\sum_{b+l+3<0} \frac{r_{1}^{\prime b+l+3}}{b+l+3} \int d \Omega_{2} n_{2}^{L} f_{b}+\ln \left(\frac{r_{1}^{\prime}}{s_{2}}\right) \int d \Omega_{2} n_{2}^{L} f_{-3-l}\right]+o\left(r_{1}^{\prime 0}\right)\right\} .
\end{aligned}
$$

We finally evaluate the difference between (5.11) and (5.13) and look for the partie finie in the sense of (5.4) (i.e., keeping the $\ln r_{1}^{\prime}$ term). We obtain

$$
(P)_{1}-P\left(\mathbf{y}_{1}\right)=\left[\ln \left(\frac{r_{1}^{\prime}}{s_{1}}\right)-1\right] \int \frac{d \Omega_{1}}{4 \pi} f_{-2} \quad(\mathrm{QED}) .
$$

The same type of result can be proved for the partie finie of the "twice-iterated" Poisson integral defined by 


$$
Q\left(\mathbf{x}^{\prime}\right)=-\frac{1}{4 \pi} \operatorname{Pf} \int d^{3} \mathbf{x}\left|\mathbf{x}-\mathbf{x}^{\prime}\right| F(\mathbf{x}) .
$$

We find, analogously to (5.5), that

$$
(Q)_{1}=-\frac{1}{4 \pi} \operatorname{Pf} \int d^{3} \mathbf{x} r_{1} F(\mathbf{x})+\left[\ln \left(\frac{r_{1}^{\prime}}{s_{1}}\right)+\frac{1}{2}\right]\left(r_{1}^{4} F\right)_{1} .
$$

For the parties finies of the gradients of the Poisson and twice-iterated Poisson integrals, we get

$$
\begin{gathered}
\left(\partial_{i} P\right)_{1}=-\frac{1}{4 \pi} \operatorname{Pf} \int d^{3} \mathbf{x} \frac{n_{1}^{i}}{r_{1}^{2}} F(\mathbf{x})+\ln \left(\frac{r_{1}^{\prime}}{s_{1}}\right)\left(n_{1}^{i} r_{1} F\right)_{1}, \\
\left(\partial_{i} Q\right)_{1}=\frac{1}{4 \pi} \operatorname{Pf} \int d^{3} \mathbf{x} n_{1}^{i} F(\mathbf{x})-\left[\ln \left(\frac{r_{1}^{\prime}}{s_{1}}\right)-\frac{1}{2}\right]\left(n_{1}^{i} r_{1}^{3} F\right)_{1} .
\end{gathered}
$$

Those results are proved in the same way as in Theorem 3 (with similar cancellations of the constants $\left.s_{1}\right)$.

\section{PARTIE-FINIE PSEUDO-FUNCTIONS}

\section{A. A class of pseudo-functions}

The concept of Hadamard partie finie of the divergent integral of functions $F \in \mathcal{F}$ yields a natural definition of a class of pseudo-functions $\operatorname{Pf} F$ ("partie finie", of $F$ ), namely linear forms on a subset of $\mathcal{F}$, of the type $G \in \mathcal{F} \rightarrow\langle\operatorname{Pf} F, G\rangle \in \mathbb{R}$, where the result of the action of $\operatorname{Pf} F$ on $G$ is denoted using a duality bracket $\langle$,$\rangle .$

Definition 5: For any function $F \in \mathcal{F}$ we define the pseudo-function $\operatorname{Pf} F$ as the linear functional which associates to any $G \in \mathcal{F}$, such that $F G=o\left(|\mathbf{x}|^{-3}\right)$ when $|\mathbf{x}| \rightarrow+\infty$, the partie-finie integral of the product FG, i.e.,

$$
\langle\operatorname{Pf} F, G\rangle=\operatorname{Pf} \int d^{3} \mathbf{x} F G,
$$

where the partie-finie integral is defined by (3.1).

As we can see, the pseudo-function $\operatorname{Pf} F$ is not a linear form on $\mathcal{F}$ itself but on the subset of $\mathcal{F}$ such that the integral converges at infinity. For simplicity's sake we will always say that statements like (6.1) are valid $\forall G \in \mathcal{F}$, without mentioning this restriction. Note also that the partie-finie integral depends on the two constants $s_{1}, s_{2} \in \mathbb{R}^{+*}$, and so is the pseudo-function which should indeed be denoted $\mathrm{Pf}_{s_{1}, s_{2}} F$. In our simplified notation we omit indicating $s_{1}$ and $s_{2}$.

An evident property of the duality bracket is its "symmetry" by exchanging the roles of the two slots of the bracket, namely,

$$
\forall(F, G) \in \mathcal{F}^{2}, \quad\langle\operatorname{Pf} F, G\rangle=\langle\operatorname{Pf} G, F\rangle .
$$

Also evident are the properties

$$
\langle\operatorname{Pf} F, G H\rangle=\langle\operatorname{Pf} G, F H\rangle=\langle\operatorname{Pf}(F G), H\rangle=\langle\operatorname{Pf}(F G H), 1\rangle .
$$

In the following we generally do not distinguish between the two slots in $\langle$,$\rangle . Accordingly we$ define the object

$$
\langle F, \operatorname{Pf} G\rangle \equiv\langle\operatorname{Pf} G, F\rangle .
$$


Even more, we allow for a bracket in which the two slots are filled with pseudo-functions. Thus, we write

$$
\langle\operatorname{Pf} F, \operatorname{Pf} G\rangle \equiv\langle\operatorname{Pf} F, G\rangle=\langle\operatorname{Pf} G, F\rangle,
$$

which constitutes merely the definition of the new object $\langle\operatorname{Pf} F, \operatorname{Pf} G\rangle$.

We denote by $\mathcal{F}^{\prime}$ the set of pseudo-functions $\operatorname{Pf} F$, when $F$ describes the class $\mathcal{F}$, introduced by Definition 5: $\mathcal{F}^{\prime}=\{\operatorname{Pf} F ; F \in \mathcal{F}\}$. Later we shall extend the definition of $\mathcal{F}^{\prime}$ to include the "limits" of some pseudo-functions. Roughly, the set $\mathcal{F}^{\prime}$ plays a role analogous to the set $\mathcal{D}^{\prime}$ in distribution theory, ${ }^{2}$ which is dual to the class $\mathcal{D}$ of functions which are both $C^{\infty}\left(\mathbb{R}^{3}\right)$ (about which we are concerned here) and zero outside a compact subset of $\mathbb{R}^{3}$. In distribution theory the set $\mathcal{D}$ is endowed with the Schwartz topology: a sequence $\left(\varphi_{n}\right)_{n \in \mathbb{N}}$ of elements of $\mathcal{D}$ converges to zero if and only if (i) $\exists n_{0} \in \mathbb{N}$ and a compact $K$ of $\mathbb{R}^{3}$ such that $\forall n \geqslant n_{0}$, $\operatorname{supp}\left(\varphi_{n}\right) \subset K$, and (ii) for any multi-index $L=i_{1} i_{2} \cdots i_{l}, \partial_{L} \varphi_{n}$ converges uniformly to zero. $\mathcal{D}^{\prime}$ is the set of linear forms on $\mathcal{D}$ that are continuous with respect to that topology. In this paper we shall not attempt to define a topology on the class $\mathcal{F}$, and shall limit ourselves (having in view the physical application) to the definition of the algebraic and differential rules obeyed by the pseudo-functions of $\mathcal{F}^{\prime}$. However we can state the following.

Lemma 3: The pseudo-functions of $\mathcal{F}^{\prime}$, when restricted to the set $\mathcal{D}$ of $C^{\infty}\left(\mathbb{R}^{3}\right)$ functions with compact support, are distributions in the sense of Schwartz:

$$
\operatorname{Pf} F_{\left.\right|_{\mathcal{D}}} \in \mathcal{D}^{\prime}
$$

Proof: All we need to check is that the pseudo-function $\operatorname{Pf} F_{\left.\right|_{\mathcal{D}}}$ is continuous with respect to the Schwartz topology. ${ }^{2}$ Consider a sequence $\varphi_{n} \in \mathcal{D}$ tending to zero in the sense recalled above. Applying the partie-finie integral in the form (3.3), we get $\left(\forall s^{\prime} \ll 1\right.$ and $\left.\forall N \in \mathbb{N}\right)$

$$
\begin{aligned}
\left\langle\operatorname{Pf} F_{\left.\right|_{\mathcal{D}}}, \varphi_{n}\right\rangle= & \int_{\mathbb{R}^{3} \backslash \mathcal{B}_{1}\left(s^{\prime}\right) \cup \mathcal{B}_{2}\left(s^{\prime}\right)} d^{3} \mathbf{x} F \varphi_{n} \\
& +\sum_{l \geqslant 0} \frac{1}{l !} \partial_{L} \varphi_{n}\left(\mathbf{y}_{1}\right)\left[\sum_{\substack{a+l+3 \leqslant N \\
\text { and } \neq 0}} \frac{s^{\prime a+l+3}}{a+l+3} \int d \Omega_{1} n_{1}^{L} f_{a}+\ln \left(\frac{s^{\prime}}{s_{1}}\right) \int d \Omega_{1} n_{1}^{L} f_{1-l-3}\right] \\
& +1 \leftrightarrow 2+o\left(s^{\prime N}\right) .
\end{aligned}
$$

Since $\varphi_{n}$ and all its derivatives $\partial_{L} \varphi_{n}$ tend uniformly towards zero in a given compact $K$, clearly so does the sequence of real numbers $\left\langle\operatorname{Pf} F_{\left.\right|_{\mathcal{D}}}, \varphi_{n}\right\rangle$, which shows that $\operatorname{Pf} F_{\left.\right|_{\mathcal{D}}}$ is indeed continuous

(QED).

Definition 6: The product (".’”) of $F \in \mathcal{F}$ and of $\operatorname{Pf} G \in \mathcal{F}^{\prime}$, and the product of two pseudofunctions $\operatorname{Pf} F$ and $\operatorname{Pf} G$, are defined as

$$
F \cdot \operatorname{Pf} G \equiv \operatorname{Pf} F \cdot \operatorname{Pf} G \equiv \operatorname{Pf}(F G) \in \mathcal{F}^{\prime} .
$$

In particular $F \cdot \operatorname{Pf} G=G \cdot \operatorname{Pf} F$.

In the following, we will remove the dot indicating the product and write indifferently

$$
F \operatorname{Pf} G=G \operatorname{Pf} F=\operatorname{Pf}(F G)=\operatorname{Pf} F \operatorname{Pf} G=F G \operatorname{Pf} 1 .
$$

Notice that from the symmetry of the duality bracket we have, $\forall H \in \mathcal{F}$,

$$
\langle G \operatorname{Pf} F, H\rangle=\operatorname{Pf} \int d^{3} \mathbf{x} F G H=\langle\operatorname{Pf} F, G H\rangle .
$$


Therefore, when applied to the restriction of pseudo-functions to $\mathcal{D}$, the product of Definition 6 agrees with the product of a distribution and a function $\psi \in C^{\infty}\left(\mathbb{R}^{3}\right)$, i.e.,

$$
\forall \varphi \in \mathcal{D}, \quad\left\langle\psi \operatorname{Pf} F_{\left.\right|_{\mathcal{D}}}, \varphi\right\rangle=\left\langle\operatorname{Pf} F_{\left.\right|_{\mathcal{D}}}, \psi \varphi\right\rangle
$$

\section{B. A Dirac delta-pseudo-function}

Consider, for $\varepsilon \in \mathbb{R}^{+*}$, the Riesz delta-function ${ }_{\varepsilon} \delta_{1}$ that we introduced in (3.8). Since ${ }_{\varepsilon} \delta_{1}$ $\in \mathcal{F}$ we can associate to it the pseudo-function $\mathrm{Pf}_{\varepsilon} \delta_{1}$. Now, Lemma 2 [see (3.9)] can be re-stated by means of the duality bracket as

$$
\lim _{\varepsilon \rightarrow 0}\left\langle\mathrm{Pf}_{\varepsilon} \delta_{1}, F\right\rangle=(F)_{1} .
$$

This motivates the following definition.

Definition 7: We define the pseudo-function $\operatorname{Pf} \delta_{1}$ by

$$
\forall F \in \mathcal{F},\left\langle\operatorname{Pf} \delta_{1}, F\right\rangle=(F)_{1} .
$$

We then extend the definition of the set $\mathcal{F}^{\prime}$ to include this pseudo-function: $\operatorname{Pf} \delta_{1} \in \mathcal{F}^{\prime}$.

Obviously $\operatorname{Pf} \delta_{1}$ can be viewed as the "limit" [but we have not defined a topology on $\mathcal{F}$ ] of the pseudo-functions $\operatorname{Pf}_{\varepsilon} \delta_{1}$ when $\varepsilon \rightarrow 0$. The restriction of $\operatorname{Pf} \delta_{1}$ to $\mathcal{D}$ is identical to the usual Dirac measure,

$$
\operatorname{Pf} \delta_{1_{\left.\right|_{\mathcal{D}}}}=\delta_{1} \equiv \delta\left(\mathbf{x}-\mathbf{y}_{1}\right),
$$

so that the pseudo-function $\operatorname{Pf} \delta_{1}$ appears as a natural generalization of the Dirac measure in the context of Hadamard parties finies. In the following, we shall do as if $\delta_{1}$ would belong to the original class of functions $\mathcal{F}$, writing, for instance,

$$
\left\langle\operatorname{Pf} F, \delta_{1}\right\rangle \equiv\left\langle\operatorname{Pf} \delta_{1}, F\right\rangle=(F)_{1} .
$$

Of course, this equation constitutes in fact the definition of the bracket $\left\langle\operatorname{Pf} F, \delta_{1}\right\rangle$.

Definition 8: For any $F \in \mathcal{F}$ the pseudo-function $\operatorname{Pf}\left(F \delta_{1}\right)$ is defined, consistently with the product (6.4), by

$$
\forall G \in \mathcal{F},\left\langle\operatorname{Pf}\left(F \delta_{1}\right), G\right\rangle=(F G)_{1} .
$$

We include into $\mathcal{F}^{\prime}$ all the pseudo-functions of this type: $\operatorname{Pf}\left(F \delta_{1}\right) \in \mathcal{F}^{\prime}$ (that is, we consider $\mathcal{F}_{\text {new }}^{\prime}=\mathcal{F}^{\prime}+\mathcal{F} \delta_{1}+\mathcal{F} \delta_{2} ;$ and we henceforth drop the "new").

Notice that an immediate consequence of the "nondistributivity" of the Hadamard partie finie, namely $(F G)_{1} \neq=(F)_{1}(G)_{1}$, is the fact that

$$
\operatorname{Pf}\left(F \delta_{1}\right) \neq(F)_{1} \operatorname{Pf} \delta_{1} .
$$

As an example, we have $\left(r_{1}\right)_{1}=0$; but $\operatorname{Pf}\left(r_{1} \delta_{1}\right)$ is not zero, since $\left\langle\operatorname{Pf}\left(r_{1} \delta_{1}\right), 1 / r_{1}\right\rangle=1$ for instance. The pseudo-function $\operatorname{Pf}\left(F \delta_{1}\right)$ represents the product of a delta-function with a function that is singular on its own support, whereas this product is ill-defined in the standard distribution theory. However, this object, as seen as a distribution, i.e., when restricted to the class $\mathcal{D}$ of smooth functions with compact support, does exist in the standard theory. Using the Taylor expansion when $r_{1} \rightarrow 0$ of any $\varphi \in \mathcal{D}$, that is $\Sigma_{l \geqslant 0}(1 / l !) r_{1}^{l} n_{1}^{L} \partial_{L} \varphi\left(\mathbf{y}_{1}\right)$, we obtain

$$
\left\langle\operatorname{Pf}\left(F \delta_{1}\right)_{\left.\right|_{\mathcal{D}}}, \quad \varphi\right\rangle=(F \varphi)_{1}=\sum_{l \geqslant 0} \frac{1}{l !} \partial_{L} \varphi\left(\mathbf{y}_{1}\right) \int \frac{d \Omega_{1}}{4 \pi} n_{1}^{L} f_{-l},
$$


where ${ }_{1} f_{-l}$ denotes the coefficient of $1 / r_{1}^{l}$ in the expansion of $F$ when $r_{1} \rightarrow 0$. Notice that the sum in (6.14) is always finite because $l \leqslant-a_{0}$, where $a_{0}=a_{0}(F)$ is the smallest exponent of $r_{1}$ in the expansion of $F$ (see Definition 1). From (6.14) we derive immediately the "intrinsic" form of the distribution $\operatorname{Pf}\left(F \delta_{1}\right)_{\left.\right|_{\mathcal{D}}}$, that is,

$$
\operatorname{Pf}\left(F \delta_{1}\right)_{\left.\right|_{\mathcal{D}}}=\sum_{l \geqslant 0} \frac{(-)^{l}}{l !} \partial_{L} \delta_{1} \int \frac{d \Omega_{1}}{4 \pi} n_{1}^{L} f_{-l}=\sum_{l \geqslant 0} \frac{(-)^{l}}{l !}\left(r_{1}^{l} n_{1}^{L} F\right)_{1} \partial_{L} \delta_{1}
$$

where $\partial_{L} \delta_{1}$ denotes the $l$ th partial derivative of the Dirac measure (and where the sums are finite). We have, for instance,

$$
\operatorname{Pf}\left(\frac{\delta_{1}}{r_{1}^{2}}\right)_{\left.\right|_{\mathcal{D}}}=\frac{1}{6} \Delta \delta_{1} .
$$

Note also that the distribution $\operatorname{Pf}\left(F \delta_{1}\right)_{\left.\right|_{\mathcal{D}}}$ can be recovered, quite naturally, from the Laplacian (in the ordinary distributional sense) of the bracket corresponding to the "Poisson" integral of $\operatorname{Pf}\left(F \delta_{1}\right)$, i.e., formed by $\operatorname{Pf}\left(F \delta_{1}\right)$ acting on the function $\mathbf{x} \rightarrow 1 /\left|\mathbf{x}-\mathbf{x}^{\prime}\right|$. For any given $\mathbf{x}^{\prime}$, this function belongs to $\mathcal{F}_{\text {loc }}$ and we are still allowed to consider such a bracket (see also Sec. V). Thus we define

$$
G\left(\mathbf{x}^{\prime}\right)=-\frac{1}{4 \pi}\left\langle\operatorname{Pf}\left(F \delta_{1}\right), \frac{1}{\left|\mathbf{x}-\mathbf{x}^{\prime}\right|}\right\rangle=-\frac{1}{4 \pi}\left(\frac{F(\mathbf{x})}{\left|\mathbf{x}-\mathbf{x}^{\prime}\right|}\right)_{1}
$$

For $\mathbf{x}^{\prime}$ different from the singularity $\mathbf{y}_{1}$, we find, using the Taylor expansion of $1 /\left|\mathbf{x}-\mathbf{x}^{\prime}\right|$ around $\mathbf{y}_{1}$,

$$
G\left(\mathbf{x}^{\prime}\right)=-\frac{1}{4 \pi} \sum_{l \geqslant 0} \frac{(-)^{l}}{l !}\left(r_{1}^{l} n_{1}^{L} F\right)_{1} \partial_{L}^{\prime}\left(\frac{1}{r_{1}^{\prime}}\right)
$$

Clearly the function $G$, if considered as a function of the variable $\mathbf{x}^{\prime}$, belongs to $\mathcal{F}$. Now, we see from (6.15) that the "ordinary" Laplacian of $G\left(\mathbf{x}^{\prime}\right)$ is precisely equal to $\operatorname{Pf}\left(F \delta_{1}\right)_{\left.\right|_{\mathcal{D}}}$, namely,

$$
\left.\Delta^{\prime} G^{\prime}\right|_{\mathcal{D}}=\sum_{l \geqslant 0} \frac{(-)^{l}}{l !}\left(r_{1}^{l} n_{1}^{L} F\right)_{1} \partial_{L}^{\prime} \delta_{1}^{\prime}=\operatorname{Pf}\left(F^{\prime} \delta_{1}^{\prime}\right)_{\left.\right|_{\mathcal{D}}}
$$

Let us point out that $G$ has no partie finie at the point $1:(G)_{1}=0$; so, in order to compute its partie finie at 1, we are not allowed to replace formally $\mathbf{x}^{\prime}$ by $\mathbf{y}_{1}$ inside the defining expression (6.17):

$$
0=-4 \pi(G)_{1} \neq\left\langle\operatorname{Pf}\left(F \delta_{1}\right), \frac{1}{r_{1}}\right\rangle=\left(\frac{F}{r_{1}}\right)_{1}=\hat{f}_{1} .
$$

[The function $G\left(\mathbf{x}^{\prime}\right)$ is not continuous at 1 , as we can easily see from its singular expansion (6.18).]

Finally let us mention how to give a sense to a pseudo-function that would be associated with the square of the delta-function. $\forall \varepsilon>0$, we have ${ }_{\varepsilon} \delta_{1}^{2} \in \mathcal{F}$, and hence, we can consider the partiefinie integral of ${ }_{\varepsilon} \delta_{1}^{2} F$. In the limit $\varepsilon \rightarrow 0$ we get

$$
\lim _{\varepsilon \rightarrow 0}\left\langle\mathrm{Pf}_{\varepsilon} \delta_{1}^{2}, F\right\rangle=\lim _{\varepsilon \rightarrow 0} \operatorname{Pf} \int d^{3} \mathbf{x}_{\varepsilon} \delta_{1}^{2} F=0
$$


essentially because we have a square $\varepsilon^{2}$ in factor which kills any divergencies arising from the integral. Therefore $\operatorname{Pf} \delta_{1}^{2}$ is (defined to be) identically zero. More generally,

$$
\forall F \in \mathcal{F}, \quad \operatorname{Pf}\left(F \delta_{1}^{2}\right)=0,
$$

and we shall not hesitate to write such identities as

$$
\left\langle\operatorname{Pf} \delta_{1}, F \delta_{1}\right\rangle=\left\langle\delta_{1}, \operatorname{Pf}\left(F \delta_{1}\right)\right\rangle=\left\langle\operatorname{Pf}\left(F \delta_{1}^{2}\right), 1\right\rangle=0 .
$$

Note also that

$$
\operatorname{Pf}\left(F \delta_{1} \delta_{2}\right)=0 .
$$

\section{DERIVATIVE OF PSEUDO-FUNCTIONS}

\section{A. A derivative operator on $\mathcal{F}$}

From now on we shall generally suppose, in order to simplify the presentation, that the powers of $r_{1}$ and $r_{2}$ in the expansions of $F \in \mathcal{F}$ around the two singularities are positive or negative integers $(\in \mathbb{Z})$. Our aim is to define an appropriate partial derivative operator acting on the pseudo-functions of the type $\operatorname{Pf} F$. First of all, we know (Lemma 3) that the restriction of $\operatorname{Pf} F$ to $\mathcal{D}$ is a distribution in the ordinary sense, so we already have at our disposal the derivative operator of distribution theory, ${ }^{2}$ which is uniquely determined - as well as any higher-order derivatives by the requirement

$$
\forall \varphi \in \mathcal{D}, \quad\left\langle\partial_{i}\left(\operatorname{Pf} F_{\left.\right|_{\mathcal{D}}}\right), \varphi\right\rangle=-\left\langle\operatorname{Pf} F_{\left.\right|_{\mathcal{D}}}, \partial_{i} \varphi\right\rangle .
$$

It is clear from viewing $\operatorname{Pf} F_{\left.\right|_{\mathcal{D}}}$ as an integral operator acting on $\varphi$, that (7.1) corresponds to a rule of "integration by part" in which the "all-integrated" (surface) term vanishes. In particular the "integral of a gradient" is zero. This motivates the following definition.

Definition 9: A partial derivative operator $\partial_{i}$ acting on pseudo-functions of $\mathcal{F}^{\prime}$ is said to satisfy the rule of integration by parts iff

$$
\forall F, G \in \mathcal{F}, \quad\left\langle\partial_{i}(\operatorname{Pf} F), G\right\rangle=-\left\langle\partial_{i}(\operatorname{Pf} G), F\right\rangle .
$$

Notice the symmetry between the two slots of the duality bracket in (7.2). As an immediate consequence, for a derivative operator satisfying this rule, we have

$$
\forall F \in \mathcal{F}, \quad\left\langle\partial_{i}(\operatorname{Pf} F), F\right\rangle=0 .
$$

Furthermore, if we assume $\partial_{i}(\operatorname{Pf} 1)=0$ in addition to Definition 9 , then

$$
\forall F \in \mathcal{F}, \quad\left\langle\partial_{i}(\operatorname{Pf} F), 1\right\rangle=0 .
$$

Of course, both (7.3) and (7.4) correspond to the intuitive idea that the integral of a gradient (in a "distributional-extended" sense) should be zero.

Proposition 3: The most general derivative operator on $\mathcal{F}^{\prime}$ satisfying the rule of integration by parts (7.2) reads as

$$
\partial_{i}(\operatorname{Pf} F)=\operatorname{Pf}\left(\partial_{i} F\right)+\mathrm{D}_{i}[F] \in \mathcal{F}^{\prime},
$$

where $\operatorname{Pf}\left(\partial_{i} F\right)$ represents the "ordinary" derivative, and where the "distributional" term $\mathrm{D}_{i}[F]=\mathrm{H}_{i}[F]+\mathrm{D}_{i}^{\text {part }}[F]$ is the sum of the general solution of the homogeneous equation, i.e., a linear functional $H_{i}[F]$ such that

$$
\forall F, G \in \mathcal{F}, \quad\left\langle\mathrm{H}_{i}[F], G\right\rangle+\left\langle\mathrm{H}_{i}[G], F\right\rangle=0,
$$


and of the particular solution defined by

$$
\mathrm{D}_{i}^{\text {part }}[F]=4 \pi \operatorname{Pf}\left(n_{1}^{i}\left[\frac{1}{2} r_{1} f_{-1}+\sum_{k \geqslant 0} \frac{1}{r_{11}^{k}} f_{-2-k}\right] \delta_{1}+1 \leftrightarrow 2\right) .
$$

When applied on any $G \in \mathcal{F}$, the particular solution reads as

$$
\left\langle\mathrm{D}_{i}^{\text {part }}[F], G\right\rangle=\int d \Omega_{1} n_{1}^{i}\left[\frac{1}{2} f_{1}^{-1} g_{-1}+\sum_{k \geqslant 0} \underset{1}{f_{-2-k} g_{k}}\right]+1 \leftrightarrow 2 .
$$

Proof: We replace the form (7.5) of the derivative operator into the rule (7.2) and find

$$
\left\langle\mathrm{D}_{i}[F], G\right\rangle+\left\langle\mathrm{D}_{i}[G], F\right\rangle=-\left\langle\operatorname{Pf}\left(\partial_{i} F\right), G\right\rangle-\left\langle\operatorname{Pf}\left(\partial_{i} G\right), F\right\rangle .
$$

The right-hand side can be readily re-written as the partie-finie integral of a gradient,

$$
\left\langle\mathrm{D}_{i}[F], G\right\rangle+\left\langle\mathrm{D}_{i}[G], F\right\rangle=-\mathrm{Pf} \int d^{3} \mathbf{x} \partial_{i}(F G) .
$$

Now we know from (3.4) that the integral of a gradient is equal to the partie finie of the surface integrals around the singularities when the surface areas shrink to zero; thus

$$
\left\langle\mathrm{D}_{i}[F], G\right\rangle+\left\langle\mathrm{D}_{i}[G], F\right\rangle=4 \pi\left(n_{1}^{i} r_{1}^{2} F G\right)_{1}+1 \leftrightarrow 2 .
$$

We replace into the right side $F$ and $G$ by their expansions around 1 , and after an easy calculation we arrive at

$$
\left\langle\mathrm{D}_{i}[F], G\right\rangle+\left\langle\mathrm{D}_{i}[G], F\right\rangle=\int d \Omega_{1} n_{1}^{i}\left[\underset{f_{-1} g_{-1}}{f_{1}}+\sum_{k \geqslant 0}\left(\begin{array}{c}
f_{-2-k} g_{k}+f_{k} g_{-2-k} \\
f_{1}
\end{array}\right)\right]+1 \leftrightarrow 2 .
$$

It is clear that the particular solution given by (7.7) or (7.8) solves the latter equation. As a consequence, the most general solution is simply obtained by adding the general solution of the homogeneous equation, i.e., (7.10) with zero on the right side, which is precisely a $\mathrm{H}_{i}[F]$ satisfying the "anti-symmetry" property $\left\langle\mathrm{H}_{i}[F], G\right\rangle+\left\langle\mathrm{H}_{i}[G], F\right\rangle=0 . \quad$ QED.

As we see from Proposition 3, the rule of integration by parts does not permit, unlike in the case of distribution theory [see (7.1)], to fully specify the derivative operator. Obviously, we must supplement the rule by another statement indicating the cases for which the new derivative should reduce to the "ordinary" one, i.e., when we should have $\partial_{i}(\operatorname{Pf} F)=\operatorname{Pf}\left(\partial_{i} F\right)$. Clearly, we would like to recover the ordinary derivative in the cases where the function is "not too much singular." In the following, we shall require essentially that our derivative reduces to the ordinary one when the function $F$ is bounded near the singularities [in addition belonging to $\left.C^{\infty}\left(\mathbb{R}^{3}-\left\{\mathbf{y}_{1,2}\right\}\right)\right]$, in the sense that there exists a neighborhood $\mathcal{N}$ containing the two singularities $\mathbf{y}_{1}$ and $\mathbf{y}_{2}$ and a constant $M \in \mathbb{R}^{+*}$ such that $\mathbf{x} \in \mathcal{N} \Rightarrow|F(\mathbf{x})| \leqslant M$. Let us refer to the coefficients of the negative powers of $r_{1}$ and $r_{2}$ in the expansions of $F$, i.e., the ${ }_{1} f_{-1-k}$ 's and ${ }_{2} f_{-1-k}$ 's where $k \in \mathbb{N}$, as the singular coefficients of $F$ (recall that we assumed that the powers of $r_{1}$ and $r_{2}$ are integers). Clearly, a function is bounded near the singularities if and only if all its singular coefficients vanish. This means that we shall require that the distributional term $\mathrm{D}_{i}[F]$, which is a linear functional of the coefficients in the expansions of $F$, should depend only on the singular coefficients ${ }_{1} f_{-1-k}$ and ${ }_{2} f_{-1-k}$ of $F$. This is already the case of our particular solution $\mathrm{D}_{i}^{\text {part }}[F]$ in (7.7). We now look for the most general possible $\mathrm{H}_{i}[F]$ depending on the ${ }_{1} f_{-1-k}$ 's (and $1 \leftrightarrow 2$ ).

All the singular coefficients admit some spherical-harmonics or equivalently STF expansions of the type (2.8)-(2.9), with STF-tensorial coefficients ${ }_{1} \hat{f}_{-1-k}^{L}$ [where $L=i_{1} \cdots i_{l}$; see (2.8) for definition], so we are led to requiring that $\mathrm{H}_{i}[F]$ be the most general (linear) functional of the STF 
tensors $\hat{f}_{1-k}^{L}$ and $1 \leftrightarrow 2$. Moreover, we demand that $\mathrm{H}_{i}[F]$, like $\mathrm{D}_{i}^{\text {part }}[F]$, is proportional to the Dirac pseudo-function $\operatorname{Pf} \delta_{1}$ (as we shall see, the gradient of $\operatorname{Pf} \delta_{1}$ is itself proportional to $\operatorname{Pf} \delta_{1}$ so there is no loss of generality). Now, we have also to take into account the fact that the dimensionality of $\mathrm{H}_{i}[F]$ should be compatible with the one of $\operatorname{Pf}\left(\partial_{i} F\right)$. Endowing $\mathbb{R}^{3}$ with a unit of length to measure the space coordinates, the Dirac pseudo-function $\operatorname{Pf} \delta_{1}$ takes the dimension of the inverse cube of a length, and $\mathrm{H}_{i}[F]$ the dimension of $F$ divided by this length (in physical applications, we do not want to introduce any special physical scale). We conclude that $\mathrm{H}_{i}[F]$ must be of the general form

$$
\mathrm{H}_{i}[F]=\sum_{k \geqslant 0} \sum_{l=0}^{+\infty} \operatorname{Pf}\left(\left[\alpha_{k, l} \hat{n}_{1}^{i L} \hat{f}_{-1-k}^{i L}+\beta_{k, l} n_{1}^{L} \hat{f}_{-1-k}^{i L}\right] r_{1}^{1-k} \delta_{1}\right)+1 \leftrightarrow 2,
$$

where the $\alpha_{k, l}$ 's and $\beta_{k, l}$ 's denote some purely constant numerical coefficients (and where, as usual, the sum over $k$ is finite). Applying this $\mathrm{H}_{i}[F]$ on any $G$ we readily obtain

$$
\left\langle\mathrm{H}_{i}[F], G\right\rangle=\sum_{k \geqslant 0} \sum_{l=0}^{+\infty} \frac{l !}{(2 l+1) ! !}\left[\frac{l+1}{2 l+3} \alpha_{k, l} \hat{f}_{-1-k}^{L} \hat{g}_{1}^{i L}{ }_{1+k}+\beta_{k, l} \hat{f}_{-1-k}^{i L} \hat{g}_{-1+k}^{L}{ }_{1}\right]+1 \leftrightarrow 2 .
$$

At last we must impose the anti-symmetry condition (7.6). For any $G$ whose all singular coefficients vanish we have $\left\langle\mathrm{H}_{i}[G], F\right\rangle=0$; then, the anti-symmetry condition tells us that (7.12) should be identically zero for any such $G$ and any $F$. Therefore, we must have $\alpha_{k, l}=0$ and $\beta_{k, l}=0$ whenever $k \geqslant 1$, so we are left with only the coefficients $\alpha_{0, l}$ and $\beta_{0, l}$, and the condition (7.6) now implies

$$
0=\sum_{l=0}^{+\infty} \frac{l !}{(2 l+1) ! !}\left(\frac{l+1}{2 l+3} \alpha_{0, l}+\beta_{0, l}\right)\left[\hat{f}_{-1}^{L} \hat{g}_{-1}^{i L}+\hat{f}_{-1}^{i L} \hat{g}_{-1}^{L}\right]+1 \leftrightarrow 2
$$

which can clearly be satisfied only if (and only if), $[(l+1) /(2 l+3)] \alpha_{0, l}+\beta_{0, l}=0$. Thus, posing $\alpha_{l} \equiv \alpha_{0, l}$, we have just proved the following.

Lemma 4: The most general $\mathrm{H}_{i}[F]$ that vanishes for any bounded function $F \in \mathcal{F}$ and possesses the correct dimension depends only on (the STF-harmonics of) the singular coefficients ${ }_{1} f_{-1}$ and ${ }_{2} f_{-1}$ and is given by

$$
\mathrm{H}_{i}[F]=\sum_{l=0}^{+\infty} \alpha_{l} \operatorname{Pf}\left(\left[\hat{n}_{1}^{i L} \hat{f}_{1}^{L}-\frac{l+1}{2 l+3} n_{1}^{L} \hat{f}_{-1}^{i L}\right] r_{1} \delta_{1}\right)+1 \leftrightarrow 2
$$

where the $\alpha_{l}$ 's form a countable set of arbitrary numerical coefficients.

[The angular dependence of the first term in (7.13) is expressed by means of the STF tensor $\hat{n}_{1}^{i L}$.] Equivalently we have

$$
\left\langle\mathrm{H}_{i}[F], G\right\rangle=\sum_{l=0}^{+\infty} \alpha \frac{(l+1) !}{(2 l+3) ! !}\left[\hat{f}_{-1}^{L} \hat{g}_{-1}^{i L}-\hat{f}_{-1}^{i L} \hat{g}_{-1}^{L}\right]+1 \leftrightarrow 2
$$

This expression is anti-symmetric in the exchange $F \leftrightarrow G$ as required.

To sum up, we have obtained the most general derivative operator $\partial_{i}(\operatorname{Pf} F)=\operatorname{Pf}\left(\partial_{i} F\right)$ $+\mathrm{D}_{i}[F]$ that satisfies the rule of integration by parts and depends only on the singular coefficients of $F$. The distributional term $\mathrm{D}_{i}[F]$ is the sum of a "particular'" solution fully specified by (7.7) or (7.8), and of a "homogeneous" solution given by (7.13) or (7.14) in terms of an infinite set of arbitrary numerical coefficients $\alpha_{l} \in \mathbb{R}$ (and $l \in \mathbb{N}$ ). In Sec. VIII we shall see how one can reduce the arbitrariness of the definition of the derivative to only one single coefficient $K \in \mathbb{R}$. 


\section{B. Some properties of the derivative}

At this stage, one can already investigate some properties of the distributional term $\mathrm{D}_{i}[F]$ $=\mathrm{D}_{i}^{\text {part }}[F]+\mathrm{H}_{i}[F]$, using the fact that the yet un-specified $\left\langle\mathrm{H}_{i}[F], G\right\rangle$ depends only on ${ }_{1} f_{-1}$ and ${ }_{1} g_{-1}$ (and $\left.1 \leftrightarrow 2\right)$. Let us first check that the derivative operator, when restricted to the smooth and compact-support functions of $\mathcal{D}$, reduces to the distributional derivative of distribution theory. ${ }^{2}$ This must actually be true since the fundamental property (7.1) of the distributional derivative is a particular case of our rule of integration by parts, and because the derivative of $\varphi \in \mathcal{D}$ reduces to the ordinary one. However, it is instructive to verify directly this fact using the expression (7.7). Applying $\mathrm{D}_{i}[F]$ on $\varphi \in \mathcal{D}$ and using the Taylor expansion of $\varphi$ around $1: \varphi$ $=\sum_{k \geqslant 0}(1 / k !) r_{1}^{k} n_{1}^{K}\left(\partial_{K} \varphi\right)\left(\mathbf{y}_{1}\right)$, we obtain

$$
\left\langle\mathrm{D}_{i}[F], \varphi\right\rangle=\sum_{k \geqslant 0} \frac{1}{k !}\left(\partial_{K} \varphi\right)\left(\mathbf{y}_{1}\right) \int d \Omega_{1} n_{1}^{i} n_{1}^{K} f_{-2-k}+1 \leftrightarrow 2 .
$$

Hence the intrinsic expression of the distributional terms on $\mathcal{D}$,

$$
\mathrm{D}_{i}[F]_{\left.\right|_{\mathcal{D}}}=\sum_{k \geqslant 0} \frac{(-)^{k}}{k !} \partial_{K} \delta_{1} \int d \Omega_{1} n_{1}^{i} n_{1}^{K} f_{-2-k}+1 \leftrightarrow 2,
$$

which agrees with the distributional part of the derivative of a function with tempered singularities in distribution theory. For example, we can write

$$
\mathrm{D}_{i}\left[\frac{1}{r_{1}^{3}}\right]_{\left.\right|_{\mathcal{D}}}=-\frac{4 \pi}{3} \partial_{i} \delta_{1}
$$

However, when acting on functions of the full set $\mathcal{F}$, the derivative generally leads to properties which have no equivalent in distributional theory. For instance, although the distributional derivative of $1 / r_{1}^{2}$ reduces on $\mathcal{D}$ to the ordinary derivative, i.e., $\mathrm{D}_{i}\left[1 / r_{1}^{2}\right]_{\left.\right|_{\mathcal{D}}}=0$, on $\mathcal{F}$ it does not:

$$
\partial_{i}\left(\operatorname{Pf} \frac{1}{r_{1}^{2}}\right)=\operatorname{Pf}\left(-2 \frac{n_{1}^{i}}{r_{1}^{3}}+4 \pi n_{1}^{i} \delta_{1}\right)
$$

For the distributional derivative of $1 / r_{1}^{3}$ on $\mathcal{F}$ we find

$$
\partial_{i}\left(\operatorname{Pf} \frac{1}{r_{1}^{3}}\right)=\operatorname{Pf}\left(-3 \frac{n_{1}^{i}}{r_{1}^{4}}+4 \pi \frac{n_{1}^{i}}{r_{1}} \delta_{1}\right) .
$$

The expression of the distributional term is apparently different from the corresponding result (7.16) in distribution theory. However we shall see after learning how to differentiate the Dirac pseudo-function $\operatorname{Pf} \delta_{1}$ that the distributional term $\mathrm{D}_{i}\left[1 / r_{1}^{3}\right]$ takes in fact the same form on $\mathcal{F}$ as on $\mathcal{D}$ [see (7.28) below].

We come now to an important point. In this paper we have defined a "pointwise" product of pseudo-functions (see Definition 6), which reduces to the ordinary product in all the cases where the functions are regular enough. For instance, it coincides with the ordinary product for $C^{\infty}$ functions, or even continuous or locally integrable functions (adopting the class $\mathcal{F}_{\text {loc }}$ ). Next, we introduced a derivative operator that acts merely as the ordinary derivative for a large class of not-too-singular functions (those which are bounded near the singularities, see Proposition 3). In particular, the derivative is equal to the ordinary one when the functions are $C^{1}$ at the location of the two singularities. However, we know from a theorem of Schwartz ${ }^{23}$ that it is impossible to define a multiplication for distributions having the previous properties and such that the distributional derivation satisfies the standard formula for the derivation of a product (Leibniz's rule). In 
agreement with that theorem, we find that the derivative operator defined by (7.5)-(7.7) does not obey in general the Leibniz rule, whereas it does satisfy it by definition in an "integrated sense," namely,

$$
\left\langle\partial_{i}[\operatorname{Pf}(F G)], 1\right\rangle=0=\left\langle\partial_{i}(\operatorname{Pf} F) G+F \partial_{i}(\operatorname{Pf} G), 1\right\rangle .
$$

However it does not satisfy the Leibniz rule in a "local sense," i.e., we have, generically for two functions $F, G \in \mathcal{F}$,

$$
\partial_{i}[\operatorname{Pf}(F G)]-\partial_{i}(\operatorname{Pf} F) G-F \partial_{i}(\operatorname{Pf} G) \neq 0 .
$$

This means that, a priori,

$$
\left\langle\partial_{i}[\operatorname{Pf}(F G)], H\right\rangle-\left\langle\partial_{i}(\operatorname{Pf} F), G H\right\rangle-\left\langle\partial_{i}(\operatorname{Pf} G), F H\right\rangle \neq 0,
$$

or, equivalently, since the Leibniz rule is satisfied by the ordinary derivative,

$$
\left\langle\mathrm{D}_{i}[F G], H\right\rangle-\left\langle\mathrm{D}_{i}[F], G H\right\rangle-\left\langle\mathrm{D}_{i}[G], F H\right\rangle \neq 0 .
$$

Actually, in accordance with the theorem in Ref. 23, (7.20) must be true even when the pseudofunction is regarded as a distribution on $\mathcal{D}$. To check this, let us compute the left side of (7.22) in the case where $\mathrm{D}_{i}$ is the particular solution $\mathrm{D}_{i}^{\text {part }}$ defined by (7.7), and where $H$ is equal to some $\varphi \in \mathcal{D}$. We employ the Taylor expansion of $\varphi$ around 1 and 2, and, strictly following the definition of the distributional term in (7.7), we arrive at

$$
\begin{aligned}
& {\left[\mathrm{D}_{i}^{\text {part }}[F G]-F \mathrm{D}_{i}^{\text {part }}[G]-G \mathrm{D}_{i}^{\text {part }}[F]\right]_{\mathcal{D}}=\sum_{k \geqslant 1} \frac{(-)^{k}}{k !} \partial_{K} \delta_{1} \int d \Omega_{1} n_{1}^{i} n_{1}^{K}\left[\frac{1}{2} f_{1} f_{1} g_{-1-k}+\frac{1}{2} f_{-1-k} g_{-1}\right.} \\
& \left.-\sum_{j=0}^{k} f_{1-1-j} g_{j-k-1}\right]+1 \leftrightarrow 2
\end{aligned}
$$

The right side of (7.23) equals $(2 \pi / 3) \partial_{i} \delta_{1}$ in the case where $F=1 / r_{1}$ and $G=1 / r_{1}^{2}$ for instance. It is not possible to add a homogeneous solution of the form (7.13) so as to always get zero. As the result (7.23) depends only on the singular coefficients of $F$ and $G$, we recover the Leibniz rule whenever $F$ or $G$ is bounded near the singularities. Besides, we can verify directly on (7.23) that the Leibniz rule is indeed true in an integrated sense, since the integral over $\mathbb{R}^{3}$ of (7.23) picks up only the term with $k=0$ which gives no contribution.

\section{Derivative of the Dirac pseudo-function}

In this subsection we compute the distributional term $\left\langle\mathrm{D}_{i}[F], G\right\rangle$ given by the sum of (7.8) and (7.14) assuming that either $F$ or $G$ is equal to the Riesz delta-function ${ }_{\varepsilon} \delta_{1}=[\varepsilon(\varepsilon$ $-1) / 4 \pi] r_{1}^{\varepsilon-3}$ for some small $\varepsilon>0$. (We come back for a moment to Definition 1 in which the powers of $r_{1}$ and $r_{2}$ in the expansions of $F$ or $G$ are real.) We notice first that the terms depending on the singular coefficients ${ }_{1} f_{-1}$ and $g_{-1}$ are present only when the exponent -1 belongs to both families of indices $\left(a_{i}\right)_{i \in \mathbb{N}}$ corresponding to $F$ and $G$ (remind Definition 1). This means that, choosing $\varepsilon$ to be different from 2, these terms will not contribute to the present calculation, and in particular that the homogeneous part $\left\langle\mathrm{H}_{i}[F], G\right\rangle$ will always give zero, provided that either $F$ or $G$ is equal to ${ }_{\varepsilon} \delta_{1}$. From the expression (7.8) we get

$$
\left\langle\mathrm{D}_{i}\left[{ }_{\varepsilon} \delta_{1}\right], G\right\rangle=\varepsilon(1-\varepsilon) \int \frac{d \Omega_{1}}{4 \pi} n_{1}^{i} g_{1-\varepsilon},
$$




$$
\left\langle\mathrm{D}_{i}[F],{ }_{\varepsilon} \delta_{1}\right\rangle=\varepsilon(1-\varepsilon) \sum_{l \geqslant 0} \frac{(-)^{l}}{l !} \partial_{1} r_{12}^{\varepsilon-3} \int \frac{d \Omega_{2}}{4 \pi} n_{2}^{i L} f_{2}-2
$$

Furthermore, by choosing $\varepsilon$ smaller than the spacing between some exponents $a_{i}$ of $G$ (specifically $\varepsilon<1-a_{i_{1}}$ with $a_{i_{1}}$ is such that $\left.a_{i_{1}}<1 \leqslant a_{i_{1}+1}\right)$ we can arrange for having ${ }_{1} g_{1-\varepsilon}=0$ so that (7.24a) becomes identically zero. Anyway, in the limit $\varepsilon \rightarrow 0$ we come up formally with both relations $\left\langle\mathrm{D}_{i}\left[\delta_{1}\right], G\right\rangle=0$ and $\left\langle\mathrm{D}_{i}[F], \delta_{1}\right\rangle=0$. The former tells us that the distributional derivative of $\operatorname{Pf} \delta_{1}$ reduces to the ordinary one, i.e.,

$$
\partial_{i}\left(\operatorname{Pf} \delta_{1}\right)=\operatorname{Pf}\left(\partial_{i} \delta_{1}\right) .
$$

The latter [that we already knew from (6.23)] shows via the rule of integration by parts that the action of $\partial_{i}\left(\operatorname{Pf} \delta_{1}\right)$ over any function $F \in \mathcal{F}$ is equal to minus the action of $\operatorname{Pf} \delta_{1}$ over the derivative $\partial_{i} F$.

Definition 10: The derivative of the Dirac pseudo-function $\operatorname{Pf} \delta_{1}$ is defined by

$$
\forall F \in \mathcal{F}, \quad\left\langle\partial_{i}\left(\operatorname{Pf} \delta_{1}\right), F\right\rangle=-\left\langle\operatorname{Pf} \delta_{1}, \partial_{i} F\right\rangle \equiv-\left(\partial_{i} F\right)_{1} .
$$

We can summarize the properties of the derivative of the Dirac pseudo-function by writing the successive identities,

$$
\left\langle\partial_{i}\left(\operatorname{Pf} \delta_{1}\right), F\right\rangle=\left\langle\operatorname{Pf}\left(\partial_{i} \delta_{1}\right), F\right\rangle=-\left\langle\operatorname{Pf} \delta_{1}, \partial_{i} F\right\rangle=-\left(\partial_{i} F\right)_{1},
$$

as well as similar identities obtained by exchanging the roles of $F$ and $\delta_{1}$,

$$
\left\langle\partial_{i}(\operatorname{Pf} F), \delta_{1}\right\rangle=\left\langle\operatorname{Pf}\left(\partial_{i} F\right), \delta_{1}\right\rangle=-\left\langle\operatorname{Pf} F, \partial_{i} \delta_{1}\right\rangle=\left(\partial_{i} F\right)_{1} .
$$

Lemma 5: The intrinsic form of the derivative of the Dirac pseudo-function is

$$
\partial_{i}\left(\operatorname{Pf} \delta_{1}\right)=-\operatorname{Pf}\left(3 \frac{n_{1}^{i}}{r_{1}} \delta_{1}\right) .
$$

The proof is evident from using the identity (2.11). The form (7.27) [with (7.25)] is quite useful in practice; for instance, it permits us to re-write the derivative of the pseudo-function $\operatorname{Pf}\left(1 / r_{1}^{3}\right)$ as computed in (7.18) into the form

$$
\partial_{i}\left(\operatorname{Pf} \frac{1}{r_{1}^{3}}\right)=\operatorname{Pf}\left(-3 \frac{n_{1}^{i}}{r_{1}^{4}}-\frac{4 \pi}{3} \partial_{i} \delta_{1}\right),
$$

where the distributional term takes the same form as in the distribution theory [compare with (7.16)].

The preceding definition and lemma are easily extended to the case of the pseudo-functions $\operatorname{Pf}\left(F \delta_{1}\right)$. The derivative of these objects is defined by the mean of the relation

$$
\left\langle\partial_{i}\left[\operatorname{Pf}\left(F \delta_{1}\right)\right], G\right\rangle=-\left\langle\operatorname{Pf}\left(F \delta_{1}\right), \partial_{i} G\right\rangle=-\left(F \partial_{i} G\right)_{1} .
$$

Then, from the identity (2.11), we readily get the intrinsic form

$$
\partial_{i}\left[\operatorname{Pf}\left(F \delta_{1}\right)\right]=\operatorname{Pf}\left[r_{1}^{3} \partial_{i}\left(\frac{F}{r_{1}^{3}}\right) \delta_{1}\right] .
$$

Notice the interesting particular case,

$$
\partial_{i}\left[\operatorname{Pf}\left(r_{1}^{3} \delta_{1}\right)\right]=0,
$$


which is also an immediate consequence of (2.16). Finally, let us mention that the Leibniz rule happens to hold in the special case where one of the pseudo-functions is of the type $\operatorname{Pf}\left(G \delta_{1}\right)$, i.e.,

$$
\partial_{i}\left[\operatorname{Pf} F \cdot \operatorname{Pf}\left(G \delta_{1}\right)\right]=\partial_{i}(\operatorname{Pf} F) \cdot \operatorname{Pf}\left(G \delta_{1}\right)+\operatorname{Pf} F . \partial_{i}\left[\operatorname{Pf}\left(G \delta_{1}\right)\right]
$$

(the verification is straightforward).

\section{MULTIPLE DERIVATIVES}

\section{A. General construction}

From Proposition 3 we can give a meaning to

$$
\left\langle\partial_{i}(\operatorname{Pf} F), G\right\rangle=\operatorname{Pf} \int d^{3} \mathbf{x} \partial_{i} F G+\left\langle\mathrm{D}_{i}[F], G\right\rangle,
$$

which will be also denoted $\left\langle\partial_{i}(\operatorname{Pf} F), \operatorname{Pf} G\right\rangle$. We now define the more complicated object $\left\langle\partial_{i}(\operatorname{Pf} F), \partial_{j}(\operatorname{Pf} G)\right\rangle$. Since the distributional term $\mathrm{D}_{i}[F]$ has the form $\operatorname{Pf}\left(H \delta_{1}\right)$ plus $1 \leftrightarrow 2$, and because (6.22)-(6.24) entail such identities as $\left\langle\operatorname{Pf}\left(G \delta_{1}\right), \operatorname{Pf}\left(H \delta_{1}\right)\right\rangle=0=\left\langle\operatorname{Pf}\left(G \delta_{1}\right), \operatorname{Pf}\left(H \delta_{2}\right)\right\rangle$, we deduce that the duality bracket applied on any two distributional terms is always zero:

$$
\forall F, G \in \mathcal{F},\left\langle\mathrm{D}_{i}[F], \mathrm{D}_{j}[G]\right\rangle=0 .
$$

When constructing the bracket $\left\langle\partial_{i}(\operatorname{Pf} F), \partial_{j}(\operatorname{Pf} G)\right\rangle$ we shall meet a product of two distributional terms which gives zero by (8.2), and we shall be left only with the ordinary part as well as the two cross terms involving one distributional term. Therefore,

$$
\left\langle\partial_{i}(\operatorname{Pf} F), \partial_{j}(\operatorname{Pf} G)\right\rangle=\operatorname{Pf} \int d^{3} \mathbf{x} \partial_{i} F \partial_{j} G+\left\langle\mathrm{D}_{i}[F], \partial_{j} G\right\rangle+\left\langle\mathrm{D}_{j}[G], \partial_{i} F\right\rangle .
$$

[The ordinary part can equivalently be written as

$$
\left.\operatorname{Pf} \int d^{3} \mathbf{x} \partial_{i} F \partial_{j} G=\left\langle\operatorname{Pf}\left(\partial_{i} F\right), \operatorname{Pf}\left(\partial_{j} G\right)\right\rangle=\left\langle\operatorname{Pf}\left(\partial_{i} F\right), \partial_{j} G\right\rangle=\left\langle\partial_{i} F, \operatorname{Pf}\left(\partial_{j} G\right)\right\rangle .\right]
$$

We now intend to introduce the second-order derivative operator. The generalization to any $l$ th-order derivative is straightforward and will be stated without proof. By extending the rule of integration by parts presented in Definition 9, we are led, quite naturally, to require that

$$
\forall F, G \in \mathcal{F},\left\langle\partial_{i j}(\operatorname{Pf} F), G\right\rangle=-\left\langle\partial_{j}(\operatorname{Pf} F), \partial_{i}(\operatorname{Pf} G)\right\rangle,
$$

where the object $\left\langle\partial_{j}(\operatorname{Pf} F), \partial_{i}(\operatorname{Pf} G)\right\rangle$ has just been given in (8.3). For the moment, we are careful at distinguishing the order of the indices $i$ and $j$. Let us look for the expression of the distributional term $\mathrm{D}_{i j}[F]$ corresponding to the double derivative, viz.,

$$
\partial_{i j}(\operatorname{Pf} F)=\operatorname{Pf}\left(\partial_{i j} F\right)+\mathrm{D}_{i j}[F],
$$

in terms of the single-derivative term $\mathrm{D}_{i}[F]$. Inserting (8.5) into the required property (8.4) we arrive immediately at

$$
\left\langle\mathrm{D}_{i j}[F], G\right\rangle=-\mathrm{Pf} \int d^{3} \mathbf{x} \partial_{i}\left(\partial_{j} F G\right)-\left\langle\mathrm{D}_{j}[F], \partial_{i} G\right\rangle-\left\langle\mathrm{D}_{i}[G], \partial_{j} F\right\rangle .
$$

Next recall the formula (7.9) which tells us that any partie-finie integral of a gradient is the sum of two distributional contributions. Using this property we obtain the simple result

$$
\left\langle\mathrm{D}_{i j}[F], G\right\rangle=\left\langle\mathrm{D}_{i}\left[\partial_{j} F\right], G\right\rangle-\left\langle\mathrm{D}_{j}[F], \partial_{i} G\right\rangle=\left\langle\mathrm{D}_{i}\left[\partial_{j} F\right], G\right\rangle+\left\langle\partial_{i} \mathrm{D}_{j}[F], G\right\rangle .
$$


The formula (7.29) allowed us to obtain the second equality; so the intrinsic form of the secondorder distributional term is obtained as

$$
\mathrm{D}_{i j}[F]=\mathrm{D}_{i}\left[\partial_{j} F\right]+\partial_{i} \mathrm{D}_{j}[F] .
$$

This result is easily extendible to any multiple derivatives, demanding that, to any order $l$,

$$
\left\langle\partial_{i_{1} i_{2} \cdots i_{l}}(\operatorname{Pf} F), G\right\rangle=-\left\langle\partial_{i_{2} \cdots i_{l}}(\operatorname{Pf} F), \partial_{i_{1}}(\operatorname{Pf} G)\right\rangle,
$$

where the right side is obtained in a way similar to (8.3). We can even impose the more general rule of integration by parts, that for any $k=1, \ldots, l$,

$$
\left\langle\partial_{i_{1} i_{2} \cdots i_{l}}(\operatorname{Pf} F), G\right\rangle=(-)^{k}\left\langle\partial_{i_{k+1} i_{k+2} \cdots i_{l}}(\operatorname{Pf} F), \partial_{i_{k} i_{k-1} \cdots i_{1}}(\operatorname{Pf} G)\right\rangle .
$$

Then the following is proved by induction over $l$.

Proposition 4: If a multi-derivative operator,

$$
\partial_{i_{1} i_{2} \cdots i_{l}}(\operatorname{Pf} F)=\operatorname{Pf}\left(\partial_{i_{1} i_{2} \cdots i_{l}} F\right)+\mathrm{D}_{i_{1} i_{2} \cdots i_{l}}[F],
$$

satisfies the rule of integration by parts (8.8) or (8.9), then the lth-order distributional term $\mathrm{D}_{i_{1} i_{2} \cdots i_{l}}[F]$ is given in terms of the first-order $\mathrm{D}_{i_{k}}[F]$ 's by

$$
\mathrm{D}_{i_{1} i_{2} \cdots i_{l}}[F]=\sum_{k=1}^{l} \partial_{i_{1} \cdots i_{k-1}} \mathrm{D}_{i_{k}}\left[\partial_{i_{k+1} \cdots i_{l}} F\right] .
$$

Recall that this result is valid for any distributional derivative of the form given by Proposition 3 , i.e., $\mathrm{D}_{i}[F]=\mathrm{D}_{i}^{\text {part }}[F]+\mathrm{H}_{i}[F]$. Therefore, the rule of integration by parts has permitted us to construct uniquely all higher-order derivatives from a given choice of first-order derivative $\mathrm{D}_{i}[F]$, i.e., from a given choice of "homogeneous" solution $\mathrm{H}_{i}[F]$. Notice that a priori this construction does not yield some commuting multi-derivatives (i.e., the Schwarz lemma is not valid in general), because evidently the right side of the formula (8.11) is not necessarily symmetric in all its indices. However, as a central result of this paper, we shall show now that it is possible to find an initial $\mathrm{H}_{i}[F]$ such that the derivatives do commute to any order.

Theorem 4: The most general derivative operator $\partial_{i}(\operatorname{Pf} F)=\operatorname{Pf}\left(\partial_{i} F\right)+\mathrm{D}_{i}[F]$ such that

(i) the distributional term $\mathrm{D}_{i}[F]$ depends only on the singular coefficients of $F$,

(ii) all multi-derivatives satisfy the rule of integration by parts,

(iii) all multi-derivatives commute (i.e., the $\mathrm{D}_{i_{1} i_{2} \cdots i_{l}}[\mathrm{~F}]$ 's are symmetric in $i_{1} i_{2} \cdots i_{l}$ ), is given by

$$
\mathrm{D}_{i}[F]=4 \pi \sum_{l=0}^{+\infty} \operatorname{Pf}\left(C_{l}\left[n_{1}^{i L} \hat{f}_{1}^{L}{ }_{1}-n_{1}^{L} \hat{f}_{-1}^{i L}\right] r_{1} \delta_{1}+\sum_{k \geqslant 0} \frac{n_{1}^{i L}}{r_{1}^{k}} \hat{f}_{-2-k}^{L} \delta_{1}\right)+1 \leftrightarrow 2,
$$

where the coefficients $C_{l}=(l+1)\left\{K+\sum_{j=1}^{l}[1 /(j+1)]\right\}$ depend on an arbitrary constant $K$.

(Actually the theorem states that the derivative operator depends a priori on two different constants $K_{1}$ and $K_{2}$ for each of the two singularities. In the following we shall assume for simplicity that the constants are the same, so that the way to differentiate does not distinguish between the different singularities.) Notice that $\mathrm{D}_{i}[F]$ differs from the particular solution $\mathrm{D}_{i}^{\text {part }}[F]$ given by (7.7) only in the terms depending on the "least singular" coefficients ${ }_{1} f_{-1}$ and ${ }_{2} f_{-1}$.

Proof: According to the assumptions (i) and (ii) we already know (see Proposition 3 and Lemma 4) that the distributional term must be of the form $\mathrm{D}_{i}[F]=\mathrm{D}_{i}^{\text {part }}[F]+\mathrm{H}_{i}[F]$, where the particular solution is given explicitly by (7.7), and where the homogeneous term takes the form (7.13) depending on a set of arbitrary coefficients $\alpha_{l}$. Furthermore, we know from Proposition 4 that all higher-order derivatives are generated from the first-order one in the way specified by 
(8.11). It only remains to show that the coefficients $\alpha_{l}$ can be computed in order that the assumption (iii) of the commutation of derivatives be fulfilled, and that the derivative is given by (8.12).

What we want then is to impose the symmetry of $\mathrm{D}_{i j}[F]$ in $i j$. We compute the antisymmetric projection $[i j] \equiv(i j-j i) / 2$ of the second-order distributional term associated with the particular solution (7.7),

$$
\mathrm{D}_{[i j]}^{\mathrm{part}}[F]=\mathrm{D}_{[i}^{\mathrm{part}}\left[\partial_{j]} F\right]+\partial_{[i} \mathrm{D}_{j]}^{\mathrm{part}}[F]
$$

The first term is readily obtained from (2.12) which tells us that the $a$ th coefficient in the $r_{1}$-expansion of the gradient is ${ }_{1} f_{a}\left(\partial_{j} F\right)=(a+1) n_{11}^{j} f_{a+1}+d_{11}^{j} f_{a+1}$. On the other hand, the second term in (8.13) comes directly from using the formula (7.30). It follows that the anti-symmetric projection depends only on the expansion coefficients ${ }_{1} f_{0},{ }_{1} f_{-1}$ and $1 \leftrightarrow 2$ through the simple formula,

$$
\mathrm{D}_{[i j]}^{\text {part }}[F]=2 \pi \operatorname{Pf}\left(n_{1}^{[i}\left[r_{1} d_{1}^{j]} f_{0}+d_{1}^{j]} f_{-1}\right] \delta_{1}\right)+1 \leftrightarrow 2
$$

or, using the relation $(2.13)$ for the operator $d_{1}^{j}$,

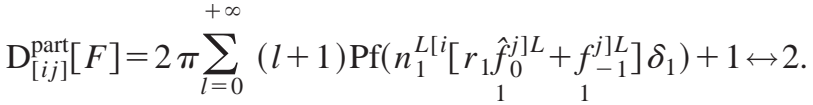

Note that by applying this on any $G$, we get

$$
\left\langle\mathrm{D}_{[i j]}^{\mathrm{part}}[F], G\right\rangle=-2 \pi \sum_{l=0}^{+\infty} \frac{(l+1)(l+1) !}{(2 l+3) ! !}\left(\hat{f}_{0}^{L[i} \hat{g}_{-1}^{j] L}+\underset{1}{{ }_{1}} \hat{f}_{1}^{L[i} \hat{g}_{0}^{j] L}\right)+1 \leftrightarrow 2 .
$$

Next, we add the homogeneous solution. By performing a computation similar as the previous one (but a bit more involved) we find, based on the expression (7.13),

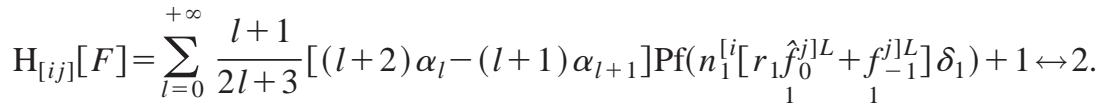

Remarkably, $\mathrm{H}_{[i j]}[F]$ takes exactly the same form as $(8.15)$. Hence, we are able to determine a relation to be satisfied by the looked-for coefficients $\alpha_{l}$ for any $l$ in order that the noncommuting part (8.15) associated to the particular solution be cancelled out by that of the homogeneous one: $\forall l,(l+2) \alpha_{l}-(l+1) \alpha_{l+1}=-2 \pi(2 l+3)$. Given any initial value for $\alpha_{0}$ the solution reads as

$$
\alpha_{l}=(l+1)\left[\alpha_{0}+2 \pi \sum_{j=1}^{l}\left(\frac{1}{j}+\frac{1}{j+1}\right)\right]=-2 \pi+4 \pi(l+1)\left[K+\sum_{j=1}^{l} \frac{1}{j+1}\right],
$$

in which we have introduced the new arbitrary constant $K=\alpha_{0} / 4 \pi+1 / 2$. Inserting (8.17) back into the expression for $\mathrm{D}_{i}[F]$ leads to the announced result (8.12). At last, we find that for any choice of the constant $K$ the second-derivative operator commutes, i.e.,

$$
\mathrm{D}_{[i j]}[F]=\mathrm{H}_{[i j]}[F]+\mathrm{D}_{[i j]}^{\mathrm{part}}[F]=0 .
$$

Let us verify from (8.18) that all higher-order multi-derivative operators commute as well, i.e., $\mathrm{D}_{i_{1} i_{2} \cdots i_{l}}[F]$ given by the formula $(8.11)$ is symmetric in all its indices. This is easily proved by induction over $l$. Suppose that to the $(l-1)$ th order $\mathrm{D}_{i_{1} i_{2} \cdots i_{l-1}}[F]$ is symmetric, and re-write the formula (8.11) into both forms,

$$
\mathrm{D}_{i_{1} i_{2} \cdots i_{l}}[F]=\mathrm{D}_{i_{1}}\left[\partial_{i_{2} \cdots i_{l}} F\right]+\partial_{i_{1}} \mathrm{D}_{i_{2} \cdots i_{l}}[F]=\mathrm{D}_{i_{1} \cdots i_{l-1}}\left[\partial_{i_{l}} F\right]+\partial_{i_{1} \cdots i_{l-1}} \mathrm{D}_{i_{l}}[F]
$$


Clearly, $\mathrm{D}_{i_{1} \cdots i_{l}}[F]$ is symmetric with respect to both $i_{1} \cdots i_{l-1}$ and $i_{2} \cdots i_{l}$, so must be symmetric in all its indices (the symmetry with respect to the first and last indices being a consequence of the other symmetries).

QED.

We should mention that the dependence upon the arbitrary constant $K$ of the derivative operator defined by Theorem 4 is

$$
\mathrm{D}_{i}[F]_{\left.\right|_{K}}=4 \pi K \sum_{l=0}^{+\infty}(l+1) \operatorname{Pf}\left(\left[n_{1}^{i L} \hat{f}_{-1}^{L}-n_{1}^{L} \hat{f}_{-1}^{i L}\right] r_{1} \delta_{1}\right)+1 \leftrightarrow 2,
$$

which can also be cast into the more interesting form

$$
\mathrm{D}_{i}[F]_{K}=-4 \pi K \partial_{i}\left[\operatorname{Pf}\left(r_{1}^{2} f_{-1} \delta_{1}\right)\right]+1 \leftrightarrow 2 .
$$

We see that the "ambiguity" linked with the constant $K$ when deriving the pseudo-function $\operatorname{Pf} F$ is related to an ambiguity resulting from the addition of the term $-4 \pi K \operatorname{Pf}\left(r_{11}^{2} f_{-1} \delta_{1}\right)+1 \leftrightarrow 2$ to $\operatorname{Pf} F$. In a sense, one can also view the constant $K$ as a measure of how much the distributional derivative of the pseudo-function $\operatorname{Pf}\left(1 / r_{1}\right)$ differs from the ordinary one, i.e.,

$$
\mathrm{D}_{i}\left[\frac{1}{r_{1}}\right]=4 \pi K \operatorname{Pf}\left(n_{1}^{i} r_{1} \delta_{1}\right) .
$$

Indeed, for functions which are more singular than a simple $1 / r_{1}$, there is no dependence on the constant $K$; see, e.g., (7.17)-(7.18).

\section{B. The Laplacian operator}

Let us compute the second-derivative of $\operatorname{Pf}\left(1 / r_{1}\right)$ using the formula $\mathrm{D}_{i j}\left[1 / r_{1}\right]=\mathrm{D}_{i}\left[-n_{1}^{j} / r_{1}^{2}\right]$ $+\partial_{i} \mathrm{D}_{j}\left[1 / r_{1}\right]$. The first term is obtained directly from the definition (8.12), and the second term is computed with the help of the formula (7.30) applied on (8.21). As a result, we get

$$
\mathrm{D}_{i j}\left[\frac{1}{r_{1}}\right]=-\frac{4 \pi}{3} \operatorname{Pf}\left(\left[\delta^{i j}+3(3 K+1) \hat{n}_{1}^{i j}\right] \delta_{1}\right),
$$

where $\hat{n}_{1}^{i j}=n_{1}^{i} n_{1}^{j}-(1 / 3) \delta^{i j}$. Evidently (because of the trace-free $\hat{n}_{1}^{i j}$ ), when we restrict ourselves to smooth functions of the set $\mathcal{D}$, we recover the usual formula of distributional theory,

$$
\mathrm{D}_{i j}\left[\frac{1}{r_{1}}\right]_{\left.\right|_{\mathcal{D}}}=-\frac{4 \pi}{3} \delta^{i j} \delta_{1} .
$$

Since the dependence over $K$ in (8.22) drops out when taking the trace over the indices $i j$, we have $\mathrm{D}_{i i}\left[1 / r_{1}\right]=-4 \pi \operatorname{Pf} \delta_{1}$ (even on the set $\mathcal{F}$ ). This means that the Laplacian of $1 / r_{1}$ on $\mathcal{F}$ takes the same form as the well-known formula of distribution theory:

$$
\Delta\left(\operatorname{Pf} \frac{1}{r_{1}}\right)=-4 \pi \operatorname{Pf} \delta_{1} .
$$

We infer from the rule of integration by parts that

$$
\left\langle\Delta(\operatorname{Pf} F), \frac{1}{r_{1}}\right\rangle=\left\langle\Delta\left(\operatorname{Pf} \frac{1}{r_{1}}\right), F\right\rangle=-4 \pi(F)_{1},
$$


which can be phrased by saying that the Poisson integral of the Laplacian of a singular function, as evaluated at a singular point, is equal to the partie finie of the function at that point. More generally, the Laplacian acting on any pseudo-function in $\mathcal{F}^{\prime}$ is defined by

$$
\Delta(\operatorname{Pf} F)=\operatorname{Pf}(\Delta F)+\mathrm{D}_{i i}[F],
$$

where the distributional term is given by

$$
\mathrm{D}_{i i}[F]=\partial_{i} \mathrm{D}_{i}[F]+\mathrm{D}_{i}\left[\partial_{i} F\right] .
$$

Proposition 5: Under the hypothesis of Theorem 4 the distributional term associated with the Laplacian operator reads as

$$
\mathrm{D}_{i i}[F]=4 \pi \sum_{l=0}^{+\infty} \operatorname{Pf}\left((l+1) C_{l-1} n_{1}^{L}\left[\hat{f}_{-1}^{L}+r_{1} \hat{f}_{0}^{L}\right] \delta_{1}-\sum_{k \geqslant 0}(2 k+1) \frac{n_{1}^{L}}{r_{1}^{k}} \hat{f}_{-1-k}^{L} \delta_{1}\right)+1 \leftrightarrow 2 .
$$

The proof is straightforward and will not be detailed. Note that the dependence on $K$ occurs only for functions owing some nonzero coefficients ${ }_{1} f_{-1}$ or ${ }_{1} f_{0}$, or $1 \leftrightarrow 2$; for instance,

$$
\begin{gathered}
\mathrm{D}_{i i}\left[n_{1}^{j}\right]=8 \pi K \operatorname{Pf}\left(n_{1}^{i} r_{1} \delta_{1}\right), \\
\mathrm{D}_{i i}\left[n_{1}^{j} / r_{1}\right]=8 \pi\left(K-\frac{1}{2}\right) \operatorname{Pf}\left(n_{1}^{i} \delta_{1}\right) .
\end{gathered}
$$

But, for more singular functions like $1 / r_{1}^{3}$, we have

$$
\Delta\left(\operatorname{Pf} \frac{1}{r_{1}^{3}}\right)=\operatorname{Pf}\left(\frac{6}{r_{1}^{5}}-\frac{20 \pi}{r_{1}^{2}} \delta_{1}\right) .
$$

Lemma 6: The Laplacian of the pseudo-function $\operatorname{Pf}\left(F \delta_{1}\right)$ is given by

$$
\Delta\left[\operatorname{Pf}\left(F \delta_{1}\right)\right]=\operatorname{Pf}\left(r_{1}^{3} \Delta\left[\frac{F}{r_{1}^{3}}\right] \delta_{1}\right) .
$$

The proof is similar to the one of the formula (7.30). Two immediate particular applications are

$$
\begin{gathered}
\Delta\left(\operatorname{Pf} \delta_{1}\right)=\operatorname{Pf}\left(\frac{6}{r_{1}^{2}} \delta_{1}\right), \\
\Delta\left[\operatorname{Pf}\left(r_{1}^{2} \delta_{1}\right)\right]=0,
\end{gathered}
$$

which can also be deduced, respectively, from (2.19) and (2.17). [(8.31a) is in agreement with (6.16).] Let us add that

$$
\Delta\left[\operatorname{Pf}\left(\frac{r_{1}}{2} \delta_{1}\right)\right]=\operatorname{Pf}\left(\frac{\delta_{1}}{r_{1}}\right) .
$$

In practice, Lemma 6 may be used to determine some solutions of Poisson equations " in the sense of distributions" on $\mathcal{F}$. For instance combining (8.31a) with the formula (8.29), we can write

$$
\Delta\left[\operatorname{Pf}\left(\frac{1}{r_{1}^{3}}+\frac{10 \pi}{3} \delta_{1}\right)\right]=\operatorname{Pf}\left(\frac{6}{r_{1}^{5}}\right),
$$

which provides a solution of the Poisson equation with source $\operatorname{Pf}\left(6 / r_{1}^{5}\right)$ in the sense of these distributions. Such a solution is by no means unique, since, from Lemma 6, one can add to it any 
"homogeneous" solution of the form $\operatorname{Pf}\left(H^{\text {hom }} \delta_{1}\right)$ where $H^{\text {hom }}$ is the product of $r_{1}^{3}$ with an arbitrary solution of the Laplace equation. Notice that (8.33) as it stands is well-defined in distribution theory and so takes the same form when restricted to $\mathcal{D}\left(\Delta \delta_{1}\right.$ is meaningful on this set). However,

$$
\Delta\left[\operatorname{Pf}\left(\frac{1}{r_{1}^{2}}+6 \pi r_{1} \delta_{1}\right)\right]=\operatorname{Pf}\left(\frac{2}{r_{1}^{4}}\right)
$$

has no equivalent in distribution theory.

\section{TIME DERIVATIVE AND PARTIAL DERIVATIVES}

The functions $F \in \mathcal{F}$ depend on the field point $\mathbf{x}$ and on the two singular source points $\mathbf{y}_{1}$ and $\mathbf{y}_{2}$. We shall now consider the situation where the two source points represent the trajectories of actual particles, and therefore depend on time $t$. We assume that the two trajectories $\mathbf{y}_{1}(t)$ and $\mathbf{y}_{2}(t)$ are smooth, that is $\mathbf{y}_{1}, \mathbf{y}_{2} \in C^{\infty}(\mathbb{R})$. In general (e.g., in the application to the problem of motion of point-particles) the function $F$ will also depend on time through the two velocities $\mathbf{v}_{1}(t)=d \mathbf{y}_{1}(t) / d t$ and $\mathbf{v}_{2}(t)=d \mathbf{y}_{2}(t) / d t$. We suppose that $F$ is a smooth functional of $\mathbf{v}_{1}$ and $\mathbf{v}_{2}$. Therefore, in this section, $F$ is supposed to take the form

$$
F=F\left(\mathbf{x}, t ; \mathbf{y}_{1}(t), \mathbf{y}_{2}(t)\right) \in \mathcal{F} .
$$

We want to investigate the partial derivatives (in a distributional sense) of the pseudo-function $\operatorname{Pf} F$ with respect to the source points $\mathbf{y}_{1}$ and $\mathbf{y}_{2}$, as well as the derivative of $\operatorname{Pf} F$ with respect to time $t$. Obviously, the partial derivatives ${ }_{1} \partial_{i} \equiv \partial / \partial \mathbf{y}_{1}$ and $1 \leftrightarrow 2$ are closely related to the time derivative $\partial_{t} \equiv \partial / \partial t$ on account of the fact that

$$
\partial_{t} F=\dot{F}+v_{1}^{i} \partial_{i} F+v_{2}^{i} \partial_{i} F
$$

(in the ordinary sense), where $\dot{F}$ denotes the contribution of the time-derivative due to the dependence over the velocities, i.e., $\dot{F}=a_{1}^{i} \partial F / \partial v_{1}^{i}+a_{2}^{i} \partial F / \partial v_{2}^{i}\left(a_{1}^{i}\right.$ and $a_{2}^{i}$ denoting the two accelerations). In applications it is frequent that $F$ depends on the trajectories only through the two distances to the field point $\mathbf{r}_{1}=\mathbf{x}-\mathbf{y}_{1}$ and $\mathbf{r}_{2}=\mathbf{x}-\mathbf{y}_{2}$; in that case,

$$
\partial_{i} F+\partial_{i} F+\partial_{2} F=0 .
$$

The general function (9.1) does not necessarily satisfy the latter identity. However, let us guess from (9.3) the result for the distributional terms ${ }_{1} \mathrm{D}_{i}[F]$ (and 1↔2) associated with the partial derivative ${ }_{1} \partial_{i}$ acting on the pseudo-function $\operatorname{Pf} F$. Since we have supposed that the dependence of $F$ on the velocities is smooth, the distributional terms will depend only on that part of the function which becomes singular when $r_{1} \rightarrow 0$, and so, because as far as the singular part is concerned, the function behaves like (9.3), the distributional terms ${ }_{1} \mathrm{D}_{i}[F]$ and ${ }_{2} \mathrm{D}_{i}[F]$ should satisfy

$$
\mathrm{D}_{i}[F]+\underset{1}{\mathrm{D}_{i}}[F]+\mathrm{D}_{i}[F]=0 .
$$

Now, from Theorem 4, we know that $\mathrm{D}_{i}[F]$ can be naturally split into two parts associated, respectively, with the singularities 1 and 2 . Therefore, we expect that the correct distributional term ${ }_{1} \mathrm{D}_{i}[F]$ is equal to minus that part of $\mathrm{D}_{i}[F]$ which corresponds to 1 . Namely, using (8.12),

$$
\mathrm{D}_{i}[F]=-4 \pi \sum_{l=0}^{+\infty} \operatorname{Pf}\left(C_{l}\left[n_{1}^{i L} \hat{f}_{-1}^{L}-n_{1}^{L} \hat{f}_{-1}^{i L}\right] r_{1} \delta_{1}+\sum_{k \geqslant 0} \frac{n_{1}^{i L}}{r_{1}^{k}} \hat{f}_{-2-k}^{L} \delta_{1}\right)
$$


(and idem for 2). This expectation is confirmed by the following definition and proposition.

Definition 11: The partial derivative ${ }_{1} \partial_{i}$ (and $1 \leftrightarrow 2$ ) acting on pseudo-functions is said to satisfy the rule of integration by parts iff

$$
\forall F, G \in \mathcal{F},\left\langle\underset{1}{\partial_{i}}(\operatorname{Pf} F), G\right\rangle+\left\langle\partial_{i}(\operatorname{Pf} G), F\right\rangle=\partial_{i}[\langle\operatorname{Pf} F, G\rangle] .
$$

Similarly, the time derivative $\partial_{t}$ is said to satisfy the rule of integration by parts iff

$$
\left\langle\partial_{t}(\operatorname{Pf} F), G\right\rangle+\left\langle\partial_{t}(\operatorname{Pf} G), F\right\rangle=\frac{d}{d t}[\langle\operatorname{Pf} F, G\rangle] .
$$

Notice that $\langle\operatorname{Pf} F, G\rangle=\operatorname{Pf} \int d^{3} \mathbf{x} F G$ is a function of the source points $\mathbf{y}_{1}(t)$ and $\mathbf{y}_{2}(t)$, as well as $t$ independently if either $F$ or $G$ depends on the velocities. The time derivative in the right side of (9.7) means the total time derivative we get by taking into account both the variable $t$ occurring through $\mathbf{y}_{1}(t)$ and $\mathbf{y}_{1}(t)$, and the independent $t$ coming from the velocities. Let us now state a result analogous to Theorem 4, whose proof will not be given since it represents a simple adaptation of the one of that theorem.

Proposition 6: Under the hypothesis of Theorem 4 the partial derivative with respect to $\mathbf{y}_{1}$ (and idem with $1 \leftrightarrow 2$ ) is determined as

$$
\partial_{i}(\operatorname{Pf} F)=\underset{1}{\operatorname{Pf}}\left(\partial_{i} F\right)+\underset{1}{\mathrm{D}_{i}}[F]
$$

where ${ }_{1} \mathrm{D}_{i}[F]$ is given by (9.5). And the time derivative is determined as

$$
\partial_{t}(\operatorname{Pf} F)=\operatorname{Pf}\left(\partial_{t} F\right)+\mathrm{D}_{t}[F]
$$

where $\mathrm{D}_{t}[F]$ is given by

$$
\mathrm{D}_{t}[F]=v_{1}^{i} \mathrm{D}_{i}[F]+v_{2}^{i} \mathrm{D}_{i}[F]
$$

Higher-order derivatives are constructed as in Sec. VIII. We find, for instance,

$$
\partial_{i j}(\operatorname{Pf} F)=\operatorname{Pf}\left(\partial_{i j} F\right)+\underset{1}{\mathrm{D}_{i}}\left[\partial_{j} F\right]+\underset{1}{\partial_{i} \mathrm{D}_{j}}[F]
$$

Idem for the second-order time derivative, which reads as

$$
\partial_{t}^{2}(\operatorname{Pf} F)=\operatorname{Pf}\left(\partial_{t}^{2} F\right)+\mathrm{D}_{t}\left[\partial_{t} F\right]+\partial_{t} \mathrm{D}_{t}[F]
$$

where $\partial_{t} F$ is given by (9.2) and $\mathrm{D}_{t}[F]$ is defined in (9.10). Furthermore the mixing up of derivatives of different type is allowed, and proceeds in the expected way. For example,

$$
\partial_{i 2} \partial_{j}(\operatorname{Pf} F)=\operatorname{Pf}\left(\partial_{i} \partial_{j} F\right)+\underset{1}{\mathrm{D}_{i}}\left[\begin{array}{l}
\left.\partial_{j} F\right] \\
\partial_{i}
\end{array}\right.
$$

Another example is

$$
\partial_{t} \partial_{i j}(\operatorname{Pf} F)=\operatorname{Pf}\left(\partial_{t} \partial_{i j} F\right)+\mathrm{D}_{t}\left[\partial_{i j} F\right]+\partial_{t} \mathrm{D}_{i}\left[\partial_{j} F\right]+\partial_{t} \partial_{i} \mathrm{D}_{j}[F]
$$

\section{ACKNOWLEDGMENTS}

The authors are grateful to Antoine Sellier for discussion and his interesting comments. This work was supported in part by the National Science Foundation under Grant No. PHY-9900776. 


\section{APPENDIX: PROOF OF THEOREM 2}

Basically the proof establishes the legitimacy of commuting some discrete series with integrals. Consider $F \in \mathcal{F}$. We start by evaluating the integrals

$$
\underset{\substack{\alpha \rightarrow 0 \\ \mathrm{FP}_{0} \rightarrow 0}}{\int_{\mathcal{B}_{1}(s)}} d^{3} \mathbf{x} \sum_{a \leqslant-3}\left(\frac{r_{1}}{s_{1}}\right)^{\alpha}\left(\frac{r_{2}}{s_{2}}\right)^{\beta} r_{1}^{a} f_{a}\left(\mathbf{n}_{1}\right)
$$

and

$$
\underset{\beta \rightarrow 0}{\mathrm{FP}_{\beta \rightarrow 0}^{\alpha \rightarrow 0}} \int_{\mathcal{B}_{1}(s)} d^{3} \mathbf{x} \sum_{a \leqslant-3}\left(\frac{r_{1}}{s_{1}}\right)^{\alpha}\left(\frac{r_{2}}{s_{2}}\right)^{\beta} r_{1}^{a} f_{a}\left(\mathbf{n}_{1}\right)
$$

where the ${ }_{1} f_{a}$ 's are the coefficients of the expansion of $F$ when $r_{1} \rightarrow 0$, and where $\mathcal{B}_{1}(s)$ is the ball centered on $\mathbf{y}_{1}$ and of radius $s \in \mathbb{R}^{+*}$ (chosen to be $s<r_{12}$ ). From the definition of the class $\mathcal{F}$ the sums over $a$ in (A1) are finite. When the real part of $\beta$ is such that $0 \leqslant \mathfrak{R}(\beta) \leqslant 1$, the integrand of (A1a) is majored by

$$
\left(\frac{r_{1}}{s_{1}}\right)^{\mathfrak{R}(\alpha)}\left(\frac{r_{2}}{s_{2}}\right)^{\mathfrak{R}(\beta)} \sum_{a \leqslant-3} r_{1}^{a}\left|f_{a}\left(\mathbf{n}_{1}\right)\right| \leqslant\left(\frac{r_{1}}{s_{1}}\right)^{\Re(\alpha)} \max \left(1, \frac{r_{2}}{s_{2}}\right) \sum_{a \leqslant-3} r_{1}^{a}\left|f_{a}\left(\mathbf{n}_{1}\right)\right|,
$$

which can be integrated on $\mathcal{B}_{1}(s)$. Thus the theorem of dominated convergence of an integral can be applied, with the result that

$$
\begin{aligned}
& \mathrm{FP}_{\substack{\alpha \rightarrow 0 \\
\alpha \rightarrow 0}} \int_{\mathcal{B}_{1}(s)} d^{3} \mathbf{x} \sum_{a \leqslant-3}\left(\frac{r_{1}}{s_{1}}\right)^{\alpha}\left(\frac{r_{2}}{s_{2}}\right)^{\beta} r_{1}^{a} f_{a}=\mathrm{FP}_{\alpha \rightarrow 0} \int_{\mathcal{B}_{1}(s)} d^{3} \mathbf{x} \sum_{a \leqslant-3}\left(\frac{r_{1}}{s_{1}}\right)^{\alpha} r_{1}^{a} f_{a} \\
& =\sum_{a+3<0} \frac{s^{a+3}}{a+3} \int d \Omega_{1} f_{a}+\ln \left(\frac{s}{s_{1}}\right) \int d \Omega_{1} f_{-3} .
\end{aligned}
$$

The second integral is more difficult to compute because the limit $\alpha \rightarrow 0$ does not commute with the integration sign. We must expand $r_{2}^{\beta}$ as a power series of $r_{1} . \forall r_{1}<r_{12}$,

$$
r_{2}^{\beta}=r_{12}^{\beta}\left(1+2 \mathbf{n}_{1} \cdot \mathbf{n}_{12} \frac{r_{1}}{r_{12}}+\frac{r_{1}^{2}}{r_{12}^{2}}\right)^{\beta / 2}=r_{12}^{\beta} \sum_{l=0}^{+\infty} C_{l}^{-\beta / 2}\left(-\mathbf{n}_{1} \cdot \mathbf{n}_{12}\right)\left(\frac{r_{1}}{r_{12}}\right)^{l},
$$

where $\mathbf{n}_{12}=\left(\mathbf{y}_{1}-\mathbf{y}_{2}\right) / r_{12}$, and where $C_{l}^{\lambda}(t)$ denotes the Gegenbauer polynomial, which is by definition the coefficient of $x^{l}$ in the power-series expansion of the function $\left(1-2 t x+x^{2}\right)^{-\lambda}$ when $x \rightarrow 0$ (with $\lambda, t \in \mathbb{C}$ ). See, e.g., Morse and Feshbach, ${ }^{27}$ p. 602. When $t \in \mathbb{R}$ and is such that $|t|$ $\leqslant 1$ (as is the case here since $t=-\mathbf{n}_{1} \cdot \mathbf{n}_{12}$ ), we can obtain a majoration of the Gegenbauer polynomial. From the formula (cf. Gradshteyn and Ryzhik, ${ }^{28}$ p. 1030)

$$
C_{l}^{\lambda}(\cos \theta)=\sum_{\substack{k, h \geqslant 0 \\ k+h=l}} \frac{\Gamma(\lambda+k) \Gamma(\lambda+h)}{k ! h ![\Gamma(\lambda)]^{2}} \cos [(k-h) \theta],
$$

we find that $\forall l \neq 0,\left|C_{l}^{\lambda}(\cos \theta)\right|$ is always less than

$$
\sum_{\substack{k, h \geqslant 0 \\ k+h=l}} \frac{(|\lambda|+k-1)(|\lambda|+k-2) \cdots|\lambda|(|\lambda|+h-1)(|\lambda|+h-2) \cdots|\lambda|}{k ! h !}=C_{l}^{|\lambda|}(1) .
$$


Therefore the series $\left.\Sigma_{l} \mid C_{l}^{-\beta / 2}\left(-\mathbf{n}_{1} \cdot \mathbf{n}_{12}\right)\right)\left(r_{1} / r_{12}\right)^{l} \mid$ is bounded by $\left(1-2 r_{1} / r_{12}+r_{1}^{2} / r_{12}^{2}\right)^{|\beta| / 2}$, and thus admits a limit. Thus (A2) converges absolutely and [when $\mathfrak{R}(\alpha)$ is large enough] the signs $\int$ and $\Sigma$ can be interchanged:

$$
\int_{\mathcal{B}_{1}(s)} d^{3} \mathbf{x} \sum_{a+3 \leqslant 0}\left(\frac{r_{1}}{s_{1}}\right)^{\alpha}\left(\frac{r_{2}}{s_{2}}\right)^{\beta} r_{1}^{a} f_{a}=\left(\frac{r_{12}}{s_{2}}\right)^{\beta} \sum_{l=0}^{+\infty} \sum_{a+3 \leqslant 0} \int_{\mathcal{B}_{1}(s)} d^{3} \mathbf{x} \frac{r_{1}^{\alpha+a+l}}{s_{1}^{\alpha} r_{12}^{l}} f_{a} C_{l}^{-\beta / 2},
$$

where $C_{l}^{-\beta / 2} \equiv C_{l}^{-\beta / 2}\left(-\mathbf{n}_{1} \cdot \mathbf{n}_{12}\right)$. We obtain the two terms

$$
\begin{gathered}
\left(\frac{r_{12}}{s_{2}}\right)^{\beta} \sum_{l=0}^{+\infty} \sum_{\substack{a+3 \leqslant 0 \\
a+l+3 \neq 0}} \frac{s^{\alpha+a+l+3}}{s_{1}^{\alpha} r_{12}^{l}(\alpha+a+l+3)} \int d \Omega_{1} f_{a} C_{l}^{-\beta / 2} \\
+\left(\frac{r_{12}}{s_{2}}\right)^{\beta} \sum_{\substack{l=0 \\
\text { finite sum }}}^{+\infty} \frac{1}{\alpha}\left(\frac{s}{s_{1}}\right)^{\alpha} \frac{1}{r_{12}^{l}} \int d \Omega_{1} f_{-l-3} C_{l}^{-\beta / 2} .
\end{gathered}
$$

The finite part when $\alpha \rightarrow 0$ of the second term reads simply as

$$
\left(\frac{r_{12}}{s_{2}}\right)^{\beta} \ln \left(\frac{s}{s_{1}}\right) \sum_{l=0}^{+\infty} \frac{1}{r_{12}^{l}} \int d \Omega_{1} f_{-l-3} C_{l}^{-\beta / 2} .
$$

On the other hand, in order to treat the first term, we must justify the commutation of the finite part with the infinite sum. Consider the series

$$
\sum_{l=0}^{+\infty} \frac{1}{\alpha+a+l+3}\left(\frac{s}{r_{12}}\right)^{l} \int d \Omega_{1} f_{a} C_{l}^{-\beta / 2} .
$$

For $\alpha$ in a disk of the complex plane centered on 0 and of radius $\epsilon$ (with $0<\epsilon<1$ ), we can bound the generic term of that series (for large enough $l$ ) by

$$
\frac{1}{|a+l+3|-\epsilon}\left(\frac{s}{r_{12}}\right)^{l}\left|\int d \Omega_{1} f_{a} C_{l}^{-\beta / 2}\right|,
$$

which is independent of $\alpha$, and whose corresponding series in $l$ converges. Therefore we can apply the limit $\alpha \rightarrow 0$ through the summation over $l$ and deduce

$$
\begin{gathered}
\lim _{\alpha \rightarrow 0}\left\{\left(\frac{r_{12}}{s_{2}}\right)^{\beta} \sum_{l=0}^{+\infty} \sum_{\substack{a+3 \leqslant 0 \\
a+l+3 \neq 0}} \frac{s^{\alpha+a+l+3}}{s_{1}^{\alpha} r_{12}^{l}(\alpha+a+l+3)} \int d \Omega_{1} f_{a} C_{l}^{-\beta / 2}\right\} \\
=\left(\frac{r_{12}}{s_{2}}\right)^{\beta} \sum_{a+3 \leqslant 0} s^{a+3} \sum_{\substack{l=0 \\
a+l+3 \neq 0}}^{+\infty} \frac{1}{a+l+3}\left(\frac{s}{r_{12}}\right)^{l} \int d \Omega_{1} f_{a} C_{l}^{-\beta / 2} .
\end{gathered}
$$

Next we apply the finite part $\operatorname{Pf}_{\beta \rightarrow 0}$ to the sum of (A3) and (A4), which involves finding the limit when $\beta \rightarrow 0$ of the series

$$
\sum_{\substack{l=0 \\ a+l+3 \neq 0}}^{+\infty} \frac{1}{a+l+3}\left(\frac{s}{r_{12}}\right)^{l} \int d \Omega_{1} f_{a} C_{l}^{-\beta / 2} .
$$

In any case the absolute value of the quantity under the $\operatorname{sign} \Sigma$ is smaller than 


$$
\left(\frac{s}{r_{12}}\right)^{l} C_{l}^{|\beta| / 2}(1) \int d \Omega_{1}\left|f_{a}\right|
$$

Furthermore we know that $C_{l}^{\lambda}(1)=\Gamma(2 \lambda+l) /[l ! \Gamma(2 \lambda)]$. For $l \neq=0, C_{l}^{|\lambda|}(1)=(2|\lambda|+l-1)$ $\times(2|\lambda|+l-2) \cdots(2|\lambda|) / l$ ! is manifestly an increasing function of $|\lambda|$, and, for $l=0, C_{0}^{|\lambda|}(1)=1$ is constant. From this we infer that $\forall l$ and for $\beta$ in the disk centered on 0 and of radius $\epsilon$, $C_{l}^{|\beta| 2}(1) \leqslant C_{l}^{\epsilon / 2}(1)$ holds, which leads to the $\beta$-independent bound

$$
\left(\frac{s}{r_{12}}\right)^{l} C_{l}^{\epsilon / 2}(1) \int d \Omega_{1}\left|f_{a}\right|
$$

which is manifestly the general term of a convergent series. Therefore the series (A5) possesses a limit when $\beta \rightarrow 0$ which is simply obtained by setting $\beta=0$ under the sign $\Sigma$. Using $C_{l}^{0}(\cos \theta)$ $=\delta_{l 0}$ we find this limit to be 0 if $a=-3$ and

$$
\frac{1}{a+3} \int d \Omega_{1} f_{a}
$$

if $a \neq=-3$. Gathering the results (A3)-(A4) and (A6), we arrive at

$$
\begin{aligned}
\sum_{a+3<0} \frac{s^{a+3}}{a+3} \int d \Omega_{1} f_{a}+\ln \left(\frac{s}{s_{1}}\right) \int d \Omega_{1} f_{-3} & =\mathrm{FP}_{\beta \rightarrow 0}^{\alpha \rightarrow 0} \int_{\mathcal{B}_{1}(s)} d^{3} \mathbf{x} \sum_{a+3 \leqslant 0}\left(\frac{r_{1}}{s_{1}}\right)^{\alpha}\left(\frac{r_{2}}{s_{2}}\right)^{\beta} r_{1}^{a} f_{a} \\
& =\mathrm{FP}_{\alpha \rightarrow 0}^{\beta \rightarrow 0} \int_{\mathcal{B}_{1}(s)} d^{3} \mathbf{x} \sum_{a+3 \leqslant 0}\left(\frac{r_{1}}{s_{1}}\right)^{\alpha}\left(\frac{r_{2}}{s_{2}}\right)^{\beta} r_{1}^{a} f_{a},
\end{aligned}
$$

from which we can now easily prove the equivalence with the Hadamard partie finie. Like in the proof of Proposition 1 we consider two open domains $\mathcal{D}_{1}$ and $\mathcal{D}_{2}$, disjoined and complementary in $\mathbb{R}^{3}$, and such that $\mathcal{B}_{1}(s) \subset \mathcal{D}_{1}$ and $\mathcal{B}_{2}(s) \subset \mathcal{D}_{2}$. We can write

$$
\int_{\mathcal{D}_{1}} d^{3} \mathbf{x}\left(\frac{r_{1}}{s_{1}}\right)^{\alpha}\left(\frac{r_{2}}{s_{2}}\right)^{\beta} F=\int_{\mathcal{D}_{1} \backslash \mathcal{B}_{1}(s)} d^{3} \mathbf{x}\left(\frac{r_{1}}{s_{1}}\right)^{\alpha}\left(\frac{r_{2}}{s_{2}}\right)^{\beta} F+\int_{\mathcal{B}_{1}(s)} d^{3} \mathbf{x}\left(\frac{r_{1}}{s_{1}}\right)^{\alpha}\left(\frac{r_{2}}{s_{2}}\right)^{\beta} F
$$

where each of the objects is defined by complex analytic continuation in a neighborhood of $\alpha$ $=\beta=0$ [the proof similar to the one after (4.1)]. Like in (4.8) we associate to $F$ the function $\widetilde{F}_{1}$ representing its "regularization", around the point 1 ,

$$
\widetilde{F}_{1}=F-\sum_{a+3 \leqslant 0} r_{1}^{a} f_{a}
$$

and we re-write the right side of (A8) as

$$
\int_{\mathcal{D}_{1} \backslash \mathcal{B}_{1}(s)} d^{3} \mathbf{x}\left(\frac{r_{1}}{s_{1}}\right)^{\alpha}\left(\frac{r_{2}}{s_{2}}\right)^{\beta} F+\int_{\mathcal{B}_{1}(s)} d^{3} \mathbf{x}\left(\frac{r_{1}}{s_{1}}\right)^{\alpha}\left(\frac{r_{2}}{s_{2}}\right)^{\beta} \widetilde{F}_{1}+\int_{\mathcal{B}_{1}(s)} d^{3} \mathbf{x}\left(\frac{r_{1}}{s_{1}}\right)^{\alpha}\left(\frac{r_{2}}{s_{2}}\right)^{\beta} \sum_{a+3 \leqslant 0} r_{1}^{a} f_{a} .
$$

Of these three terms, the first two are well-defined when $\alpha$ and $\beta$ tend to zero, hence their finite parts are simply obtained by posing $\alpha=0=\beta$. On the other hand we have proved previously that the finite parts $\operatorname{Pf}_{\substack{\alpha \rightarrow 0 \\ \beta \rightarrow 0}}$ and $\mathrm{Pf}_{\substack{\alpha \rightarrow 0 \\ \alpha \rightarrow 0}}$ of the third term are equal and we have found their common value to be given by (A7). This shows immediately that 


$$
\begin{aligned}
\mathrm{FP}_{\substack{\alpha \rightarrow 0 \\
\beta \rightarrow 0}} \int_{\mathcal{D}_{1}} d^{3} \mathbf{x}\left(\frac{r_{1}}{s_{1}}\right)^{\alpha}\left(\frac{r_{2}}{s_{2}}\right)^{\beta} F= & \int_{\mathcal{D}_{1} \backslash \mathcal{B}_{1}(s)} d^{3} \mathbf{x} F+\int_{\mathcal{B}_{1}(s)} d^{3} \mathbf{x} \widetilde{F}_{1} \\
& +\sum_{a+3<0} \frac{s^{a+3}}{a+3} \int d \Omega_{1} f_{a}+\ln \left(\frac{s}{s_{1}}\right) \int d \Omega_{1} f_{-3}
\end{aligned}
$$

(and idem with $\mathrm{Pf}_{\alpha \rightarrow 0}^{\beta \rightarrow 0}$ ). We recognize on the right side of (A10) the Hadamard partie finie of the integral. Indeed the second term clearly admits an expansion in positive powers of $s$,

$$
\forall N \in \mathbb{N}, \quad \int_{\mathcal{B}_{1}(s)} d^{3} \mathbf{x} \widetilde{F}_{1}=\sum_{0<a+3 \leqslant N} \frac{s^{a+3}}{a+3} \int d \Omega_{1} f_{a}+o\left(s^{N}\right),
$$

so we recover exactly the partie-finie integral over $\mathcal{D}_{1}$ in the form given by (3.3).

QED.

${ }^{1}$ J. Hadamard, Le Problème de Cauchy et Les équations aux Dérivées Partielles Linéaires Hyperboliques (Hermann, Paris, 1932).

${ }^{2}$ L. Schwartz, Théorie des Distributions (Hermann, Paris, 1978).

${ }^{3}$ R. Estrada and R. P. Kanwal, Proc. R. Soc. London, Ser. A 401, 281 (1985).

${ }^{4}$ R. Estrada and R. P. Kanwal, J. Math. Anal. Appl. 141, 195 (1989).

${ }^{5}$ A. Sellier, Proc. R. Soc. London, Ser. A 445, 69 (1964).

${ }^{6}$ D. S. Jones, Math. Methods Appl. Sci. 19, 1017 (1996).

${ }^{7}$ L. Bel, T. Damour, N. Deruelle, J. Ibañez, and J. Martin, Gen. Relativ. Gravit. 13, 963 (1981).

${ }^{8}$ T. Damour and N. Deruelle, Phys. Lett. A 87, 81 (1981).

${ }^{9}$ G. Schäfer, Ann. Phys. (N.Y.) 161, 81 (1985).

${ }^{10}$ L. Blanchet, T. Damour, and B. R. Iyer, Phys. Rev. D 51, 5360 (1995).

${ }^{11}$ P. Jaranowski, in Mathematics of Gravitation, edited by A. Królak (Banach Center Publications, 1997).

${ }^{12}$ P. Jaranowski and G. Schäfer, Phys. Rev. D 57, 7274 (1998).

${ }^{13}$ L. Blanchet, G. Faye, and B. Ponsot, Phys. Rev. D 58, 124002 (1998).

${ }^{14}$ T. Damour, in Gravitational Radiation, edited by N. Deruelle and T. Piran (North-Holland, New York, 1983).

${ }^{15}$ S. M. Kopejkin, Astron. Zh. 62, 889 (1985).

${ }^{16}$ L. P. Grishchuk and S. M. Kopejkin, in Relativity in Celestial Mechanics and Astrometry, edited by J. Kovalevsky and V. A. Brumberg (Reidel, Dordrecht, 1986).

${ }^{17}$ L. Blanchet and G. Faye, Phys. Lett. A 271, 58 (2000).

${ }^{18}$ C. Cutler, T. A. Apostolatos, L. Bildsten et al., Phys. Rev. Lett. 70, 2984 (1993).

${ }^{19}$ I. M. Gel'fand and G. E. Shilov, Generalized Functions (Academic, New York, 1964).

${ }^{20}$ D. S. Jones, Generalized Functions (Cambridge University Press, Cambridge, 1982).

${ }^{21}$ R. P. Kanwal, Generalized Functions, Theory and Technique (Academic, New York, 1983).

${ }^{22}$ M. Riesz, Acta Math. 81, 1 (1949).

${ }^{23}$ L. Schwartz, C. R. Acad Sci. Paris 239, 847 (1954).

${ }^{24}$ J. F. Colombeau, J. Math. Anal. Appl. 94, 96 (1983).

${ }^{25}$ K. S. Thorne, Rev. Mod. Phys. 52, 299 (1980).

${ }^{26}$ L. Blanchet and T. Damour, Philos. Trans. R. Soc. London, Ser. A 320, 379 (1986).

${ }^{27}$ M. P. Morse and H. Feshbach, Methods of Theoretical Physics (McGraw-Hill, New York, 1953).

${ }^{28}$ I. S. Gradshteyn and I. M. Ryzhik, Table of Integrals, Series and Products (Academic, New York, 1980). 